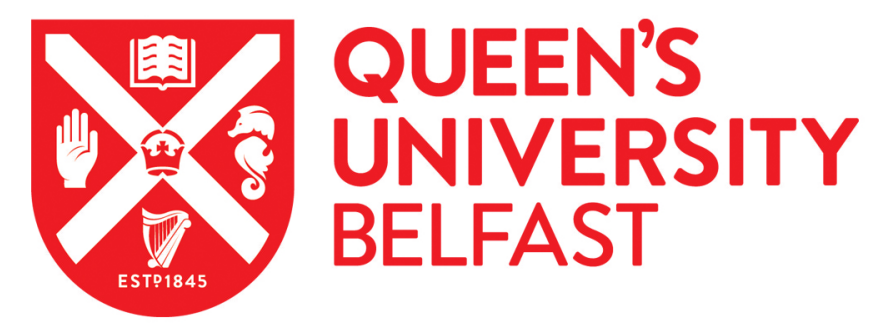

\title{
Catalyst-free room-temperature iClick reaction of molybdenum(II) and tungsten(II) azide complexes with electron-poor alkynes: Structural preferences and kinetic studies
}

Schmid, P., Maier, M., Pfeiffer, H., Belz, A., Henry, L., Friedrich, A., Schonfeld, F., Edkins, K., \&

Schatzschneider, U. (2017). Catalyst-free room-temperature iClick reaction of molybdenum(II) and tungsten(II) azide complexes with electron-poor alkynes: Structural preferences and kinetic studies. Dalton Transactions. https://doi.org/10.1039/C7DT03096G

Published in:

Dalton Transactions

Document Version:

Peer reviewed version

Queen's University Belfast - Research Portal:

Link to publication record in Queen's University Belfast Research Portal

Publisher rights

(C) The Royal Society of Chemistry 2017. This work is made available online in accordance with the publisher's policies. Please refer to any applicable terms of use of the publisher.

\section{General rights}

Copyright for the publications made accessible via the Queen's University Belfast Research Portal is retained by the author(s) and / or other copyright owners and it is a condition of accessing these publications that users recognise and abide by the legal requirements associated with these rights.

\section{Take down policy}

The Research Portal is Queen's institutional repository that provides access to Queen's research output. Every effort has been made to ensure that content in the Research Portal does not infringe any person's rights, or applicable UK laws. If you discover content in the

Research Portal that you believe breaches copyright or violates any law, please contact openaccess@qub.ac.uk. 


\section{Dalton \\ Transactions}

\section{Catalyst-free room-temperature iClick reaction of molybdenum(II) and tungsten(II) azide complexes with electron-poor alkynes: Structural preferences and kinetic studies}

\begin{tabular}{|c|c|}
\hline Journal: & Dalton Transactions \\
\hline Manuscript ID & DT-ART-08-2017-003096.R1 \\
\hline Article Type: & Paper \\
\hline Date Submitted by the Author: & $\mathrm{n} / \mathrm{a}$ \\
\hline Complete List of Authors: & $\begin{array}{l}\text { Schmid, Paul; Julius-Maximilians-Universität Würzburg, Institut für } \\
\text { Anorganische Chemie } \\
\text { Maier, Matthias; Julius-Maximilians-Universität Würzburg, Institut für } \\
\text { Anorganische Chemie } \\
\text { Pfeiffer, Hendrik; Julius-Maximilians-Universität Würzburg, Institut für } \\
\text { Anorganische Chemie } \\
\text { Belz, Anja; Julius-Maximilians-Universität Würzburg, Institut für } \\
\text { Anorganische Chemie } \\
\text { Henry, Lucas; Julius-Maximilians-Universität Würzburg, Institut für } \\
\text { Anorganische Chemie } \\
\text { Friedrich, Alexandra; Julius-Maximilians-Universität Würzburg, Institut für } \\
\text { Anorganische Chemie } \\
\text { Schönfeld, Fabian; Julius-Maximilians-Universität Würzburg, Institut für } \\
\text { Anorganische Chemie } \\
\text { Edkins, Katharina; Queen's University Belfast, School of Pharmacy; } \\
\text { Schatzschneider, Ulrich; Julius-Maximilians-Universität Würzburg, Institut } \\
\text { für Anorganische Chemie }\end{array}$ \\
\hline
\end{tabular}




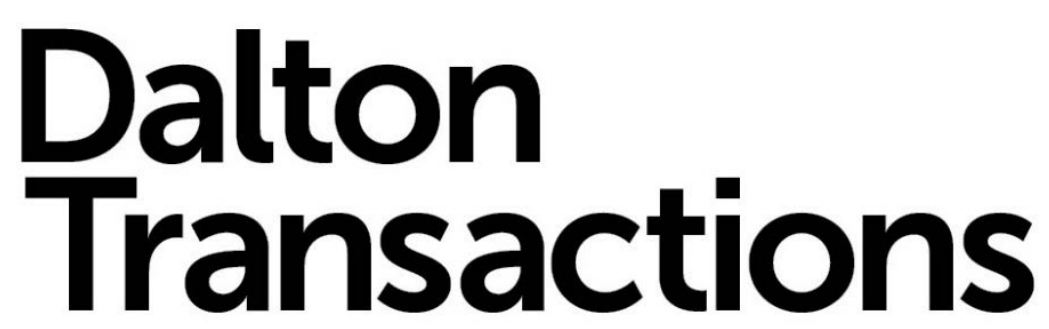

\section{Guidelines to Referees}

Communications \& Papers

The international journal for high quality, original research in inorganic and organometallic chemistry

\section{Dalton Transactions}

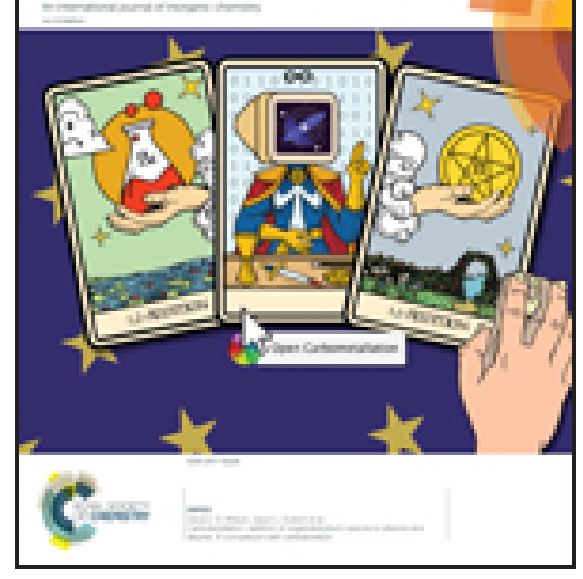

\section{http://www.rsc.org/dalton}

Dalton Transactions wishes to encourage high quality articles reporting exciting new developments in inorganic chemistry.

For an article to be accepted, it must report new, high-quality research and make a significant contribution to the field.

Manuscripts that describe purely physical, crystallographic or computational studies must include the clear relevance of the work to the broad inorganic chemistry readership of Dalton Transactions.

Communications must report chemistry of sufficient importance and impact to justify preliminary publication. Papers should report more complete studies.

\section{Dalton Transactions' Impact Factor is 4.029 (2016 Journal Citation Reports ${ }^{\circledR}$ )}

Routine or unnecessarily fragmented work, however competently researched and reported, should not be recommended for publication.

Thank you very much for your assistance in evaluating this manuscript

Dr Andrew Shore (dalton@rsc.org)

Royal Society of Chemistry

Editor, Dalton Transactions
Professor John Arnold University of California, Berkeley Chair, Dalton Transactions Editorial Board

\section{General Guidance (for further details, see the RSC Refereeing Procedure and Policy)}

When preparing your report, please:

- Comment on the originality, importance, impact and scientific reliability of the work

- State clearly whether you would like to see the paper accepted or rejected and give detailed comments (with references, as appropriate) that will help both the Editor to make a decision on the paper and the authors to improve it

Please inform the Editor if:

- There is a conflict of interest

- There is a significant part of the work which you are not able to referee with confidence

- If the work, or a significant part of the work, has previously been published, including online publication

(e.g. on a preprint server/open access server)

- You believe the work, or a significant part of the work, is currently submitted elsewhere

- The work represents part of an unduly fragmented investigation 


\title{
Journal Name
}

\section{ARTICLE}

\section{Catalyst-free room-temperature iClick reaction of molybdenum(II) and tungsten(II) azide complexes with electron-poor alkynes: Structural preferences and kinetic studies}

Received 00th January 20xx Accepted 00th January 20xx

DOI: $10.1039 / \times 0 \times x 00000 x$

www.rsc.org/

Paul Schmid, ${ }^{a}$ Matthias Maier, ${ }^{a}$ Hendrik Pfeiffer, ${ }^{a}$ Anja Belz, ${ }^{a}$ Lucas Henry, ${ }^{a}$ Alexandra Friedrich, ${ }^{a}$ Fabian Schönfeld, ${ }^{a}$ Katharina Edkins ${ }^{a, b}$ and Ulrich Schatzschneider ${ }^{a^{*}}$

\begin{abstract}
Two isostructural and isoelectronic group $\mathrm{VI}$ azide complexes of the general formula $\left[\mathrm{M}\left(\eta^{3}\right.\right.$-allyl)( $\left.\left(\mathrm{N}_{3}\right)(\mathrm{bpy})(\mathrm{CO})_{2}\right]$ with $\mathrm{M}=$ Mo, $\mathrm{W}$ and bpy $=2,2^{\prime}$-bipyridine were prepared and fully characterized, including $\mathrm{X}$-ray structure analysis. Both reacted smoothly with electron-poor alkynes such as dimethyl acetylenedicarboxylate (DMAD) and 4,4,4-trifluoro-2-butynoic acid ethyl ester in a catalyst-free room-temperature iClick [3+2] cycloaddition reaction. Reaction with phenyltrifluoromethylacetylene, on the other hand, did not lead to any product formation. X-ray structures of the four triazolate complexes isolated showed the monodentate ligand to be N2-coordinated in all cases, which requires a 1,2-shift of the nitrogen from the terminal azide to the triazolate cycloaddition product. On the other hand, a ${ }^{19} \mathrm{~F}$ NMR spectroscopic study of the reaction of the fluorinated alkyne with the tungsten azide complex at $27^{\circ} \mathrm{C}$ allowed detection of the N1-coordinated intermediate. With this method, the second-order rate constant was determined as $(7.3 \pm 0.1) \times 10^{-2} \mathrm{M}^{-1} \mathrm{~s}^{-1}$, which compares favorably with that of first-generation compounds such as difluorocyclooctyne (DIFO) used in the strainpromoted azide-alkyne cycloaddition (SPAAC). In contrast, the reaction of the molybdenum analogue was too fast to be studied with NMR methods. Alternatively, solution IR studies revealed pseudo-first order rate constants of 0.4 to $6.5 \times 10^{-3}$ $\mathrm{s}^{-1}$, which increased in the order of $\mathrm{Mo}>\mathrm{W}$ and $\mathrm{F}_{3} \mathrm{C}-\mathrm{C}=\mathrm{C}-\mathrm{COOEt}>\mathrm{DMAD}$.
\end{abstract}

\section{Introduction}

Since their introduction about 15 years ago, ${ }^{1}$ "click" reactions have been an indispensable tool for drug development ${ }^{2-4}$ and also attracted considerable attention in bioorthogonal labelling. ${ }^{5-8}$ Careful optimization of the reactants has led to systems with very fast coupling kinetics ( $k$ up to $\left.10^{5} \mathrm{M}^{-1} \mathrm{~s}^{-1}\right),{ }^{9,10}$ which has enabled the study of cellular processes on a timescale of minutes. ${ }^{11}$ In recent years, interest has also been directed at the development of inorganic "click" (iClick) reactions which involve small ligands directly coordinated to a metal center. ${ }^{12,13}$ In particular, the [3+2] cycloaddition reaction of metal-azide complexes with terminal and internal alkynes as well as nitriles gives rise to metal-triazolate and metal-tetrazolate complexes, respectively. ${ }^{14-16}$ Very recently, dual-functional platinum(II) complexes incorporating both a metal-coordinated alkyne and a peripheral organoazide moiety have also been sucessfully polymerized to metallopoly-

\footnotetext{
a. Institut für Anorganische Chemie, Julius-Maximilians-Universität Würzburg, Am Hubland, D-97074 Würzburg, Germany, +49 9313183636

ulrich.schatzschneider@uni-wuerzburg.de.

${ }^{b .}$ School of Pharmacy, Queen's University Belfast, 97 Lisburn Road, Belfast BT9 7BL, United Kingdom

Electronic Supplementary Information (ESI) available: Synthesis of ligand and metal complex precursors; molecular structures of 2, 5, 6, and 11; crystallographic parameters for all X-ray structures; IR spectra of precursors and kinetic traces; DFTcalculated conformational energy diagramm of 6 . See DOI: 10.1039/x0xx00000x
}

triazolates, however under addition of copper(II) acetate as a catalyst. ${ }^{17}$ While initial kinetic studies by Veige et al. were focused on linear gold(I) azide compounds, ${ }^{18}$ we have recently shown for a series of $\mathrm{Cp}^{*} \mathrm{Rh}(\mathrm{III})$ azide complexes with substituted 2,2'-bipyridine (bpy) coligands that the rate of the reaction is accelerated by introduction of electron-donating groups in the 4- and 4'-position of the bpy. ${ }^{19}$ However, the effect of variation of metal center and alkyne on the speed of the iClick reaction has not been investigated so far. Herein, we report on the synthesis and structural characterization of two isoelectronic molybdenum(II) and tungsten(II) azide complexes $\left[\mathrm{M}\left(\eta^{3}\right.\right.$-allyl $\left.)\left(\mathrm{N}_{3}\right)(\mathrm{bpy})(\mathrm{CO})_{2}\right]$ and their iClick cycloaddition reaction with two different electron-deficient alkynes, dimethyl acetylenedicarboxylate (DMAD) and 4,4,4-trifluoro-2butynoic acid ethyl ester, which are formally related to each other by substitution of a methyl ester by a trifluoromethyl group. In particular, ${ }^{19} \mathrm{~F}$ NMR studies involving the latter coupling partner were aimed at identifying the initial N1bound triazolate species which should form from the terminal azide ligand in the starting material, while essentially all iClick products with substituents in the 4- and 5-position of the triazolate ring structurally characterized so far are N2coordinated, ${ }^{20-28}$ with some rare N1-bound structures possibly enforced by increased steric bulk on the substituents. ${ }^{29}$

\section{Results and discussion}




\section{Synthesis}

The azide complexes $\left[\mathrm{M}\left(\eta^{3}\right.\right.$-allyl $\left.)\left(\mathrm{N}_{3}\right)(\mathrm{bpy})(\mathrm{CO})_{2}\right]$ with $\mathrm{M}=\mathrm{Mo}$ (2) and $\mathrm{W}$ (10) were prepared from the corresponding halides 1 and 9, respectively. In the case of the molybdenum compound, a one-pot reaction of $\left[\mathrm{Mo}(\mathrm{CO})_{6}\right]$ with allyl chloride and 2,2'-bipyridine in tetrahydrofuran afforded 1 in 76\% yield. ${ }^{30}$ However, the corresponding tungsten complex was not accessible via this route due to considerably slower ligand exchange on the $5 \mathrm{~d}$ vs. $4 \mathrm{~d}$ metal center. Therefore, a consecutive reaction sequence had to be employed. Starting from $\left[\mathrm{W}(\mathrm{CO})_{6}\right]$, two carbonyl ligands were oxidized to carbon dioxide by addition of 2 eq. of trimethylamine $\mathrm{N}$-oxide (TMAO), ${ }^{31}$ which facilitates the introduction of the 2,2'bipyridine ligand under mild conditions, leading to $\left[\mathrm{W}(\mathrm{bpy})(\mathrm{CO})_{4}\right](7)$, although only in a low efficiency of $31 \%$. An additional carbonyl ligand was then replaced by pyridine upon reflux in xylene for $18 \mathrm{~h}$, leading to $\left[\mathrm{W}(\mathrm{bpy})(\mathrm{CO})_{3}(\mathrm{py})\right](8)$ in $76 \%$ yield. $^{32}$ The mixed-ligand bipyridine/pyridine complex then underwent oxidative addition of allyl chloride to give $\left[\mathrm{W}\left(\eta^{3}\right.\right.$-allyl $\left.) \mathrm{Cl}(\mathrm{bpy})(\mathrm{CO})_{2}\right](9)$ in a yield of $86 \%$. Introduction of the azide ligands also required different procedures. In the case of molybdenum, the chlorido complex $\mathbf{1}$ was first treated with silver triflate to precipitate the halide as insoluble silver chloride, followed by reaction with a 2 -fold excess of sodium azide in acetonitrile at room temperature overnight, to give $\mathbf{2}$ in $58 \%$ yield. The analogous tungsten compound, on the other hand, was obtained by refluxing chlorido complex 9 in a mixture of acetone/methanol in the presence of 5 eq. of sodium azide for $6 \mathrm{~h}$, which allowed isolation of 10 in $62 \%$ yield.

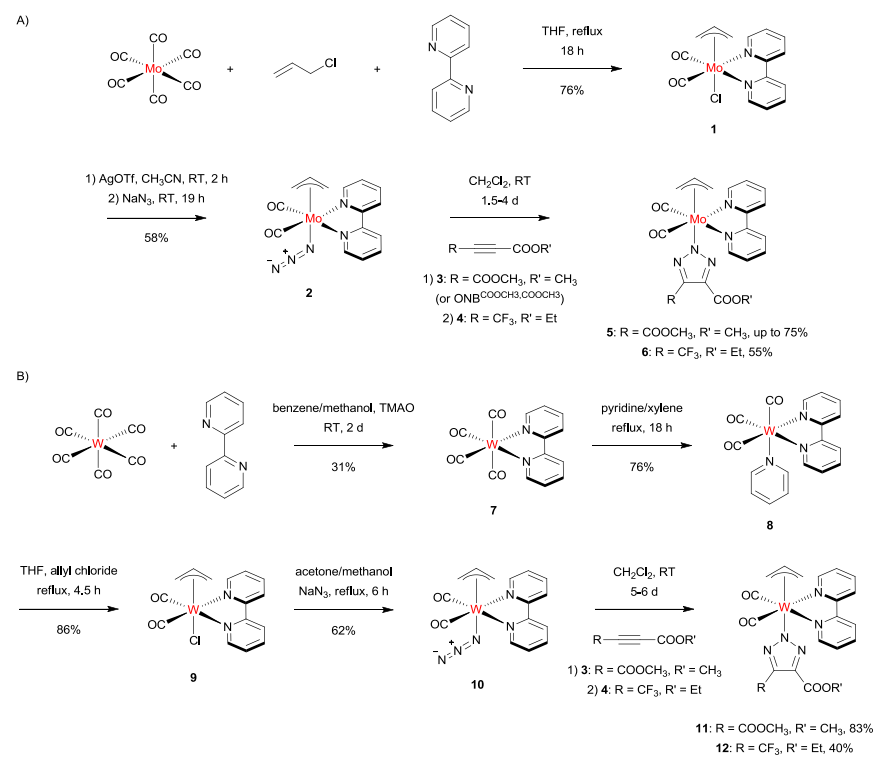

Scheme 1 Synthesis of molybdenum and tungsten triazolate complexes 5, 6, 11, and $\mathbf{1 2}$ by catalyst-free room temperature "iClick" reaction of the corresponding azide compounds with electron poor alkynes dimethyl acetylenedicarboxylate (DMAD, 3) and 4,4,4-trifluoro-2-butynoic acid ethyl ester (4) or "masked" alkyne dimethyl-7-oxa-

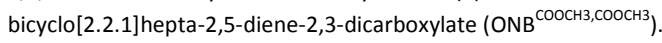

The molybdenum and tungsten azide compounds $\mathbf{2}$ and $\mathbf{1 0}$ were then tested for their efficiency in the catalyst-free "iclick" cycloaddition reaction with electron-poor alkynes dimethyl acetylenedicarboxylate (DMAD, 3) and 4,4,4-trifluoro-2-butynoic acid ethyl ester (4). Using these coupling partners, the [3+2] cycloaddition reaction proceeded smoothly at room temperature in dichloromethane, from which the triazolate complexes 5, 6, 11, and 12 were easily isolated by precipitation with diethyl ether or $n$-hexane in moderate to good yield (36-85\%). Alternatively, a "masked" alkyne in the form of dimethyl-7-oxa-bicyclo[2.2.1] hepta2,5-diene-2,3-dicarboxylate $\left(\mathrm{ONB}^{\mathrm{COOCH} 3, \mathrm{COOCH} 3}\right)$ was also utilized in the cycloaddition with $\mathbf{2}^{33}$ which gave an even higher yield of triazolate $\mathbf{5}$ compared to the reaction with DMAD (3). To further evaluate the scope of dipolarophiles, phenyltrifluoromethylacetylene (15) was prepared in a three-step published procedure with the pyrolysis of triphenylphosphonium- $\alpha$-(trifluoroacetyl)benzylide (14) as the key step (Scheme S1). ${ }^{34-40}$ However, no product could be isolated from a mixture of molybdenum azide complex $\mathbf{2}$ and alkyne $\mathbf{1 5}$ upon reaction in dichloromethane at room temperature for $24 \mathrm{~h}$. A change of the solvent to acetone and increase of the reaction temperature to reflux did not improve the outcome and in both cases, the precipitated red solid turned out to be the molybdenum azide starting material 2 based on ${ }^{1} \mathrm{H}$ and ${ }^{19} \mathrm{~F}$ NMR analysis. Either the presence of only one electron-withdrawing group is insufficient for a proper match of frontier orbitals or the phenyl group is sterically too demanding to allow approach to the azide reaction partner.

\section{Spectroscopy and X-ray structure analysis}

The strong and well-separated vibrational bands of the azide, carbonyl, and triazolate ester groups provide an easy handle for the identification of the reaction products with IR spectroscopy. In particular, the successful substitution of the chlorido by azide ligand is evident from the strong azide antisymmetric stretch present in $\mathbf{2}$ and $\mathbf{1 0}$ at 2036 and 2046 $\mathrm{cm}^{-1}$, respectively (Table 1$) .{ }^{41}$ In the course of the iClick cycloaddition reaction with alkynes, $v_{\text {asym }}\left(\mathrm{N}_{3}\right)$ disappears and instead, the $\mathrm{C}=\mathrm{O}$ stretches of the ester carbonyl groups on the triazolate ring formed are observed at about 1710 to 1725 $\mathrm{cm}^{-1}$.

Table 1 Comparison of azide, carbonyl, and triazolate ester group vibrations for molybdenum and tungsten complexes $\left[\mathrm{M}\left(\eta^{3}\right.\right.$-allyl)X(bpy) $\left.(\mathrm{CO})_{2}\right] \mathbf{1}, \mathbf{2}, \mathbf{5}$, and $\mathbf{6}$ as well as 9-12 (in $\left.\mathrm{cm}^{-1}\right)$.

\begin{tabular}{|c|c|c|c|}
\hline$x$ & molybdenum & tungsten & $\begin{array}{c}\text { difference } \\
v(\mathrm{~W})-v(\mathrm{Mo})\end{array}$ \\
\hline \multirow{2}{*}{$\mathrm{Cl}$} & $1925(\mathrm{C} \equiv 0)$ & 1915 (C三O) & -10 \\
\hline & $1832(\mathrm{C} \equiv 0)$ & 1819 (C三O) & -13 \\
\hline \multirow{3}{*}{$\mathrm{N}_{3}$} & $2036\left(N_{3}\right)$ & $2046\left(N_{3}\right)$ & +10 \\
\hline & 1928 (C三O) & 1920 (C三O) & -8 \\
\hline & $1836(\mathrm{C} \equiv \mathrm{O})$ & $1821(\mathrm{C} \equiv 0)$ & -15 \\
\hline \multirow{3}{*}{ triazolate ${ }^{\mathrm{COOCH}, \mathrm{COOCH} 3}$} & 1931 (C三O) & 1921 (C三O) & -10 \\
\hline & $1856(\mathrm{C} \equiv 0)$ & $1841(\mathrm{C} \equiv 0)$ & -15 \\
\hline & $1723(\mathrm{C}=\mathrm{O})$ & $1724(\mathrm{C}=0)$ & +1 \\
\hline \multirow{3}{*}{ triazolate ${ }^{\mathrm{CF}, \mathrm{COOEt}}$} & 1938 (C三O) & 1929 (C三O) & -9 \\
\hline & $1864,1854(\mathrm{C} \equiv 0)$ & $1850(C \equiv 0)$ & -9 \\
\hline & $1713(C=0)$ & $1723(C=0)$ & +10 \\
\hline
\end{tabular}

The symmetric and antisymmetric stretches of the cis- $\mathrm{M}(\mathrm{CO})_{2}$ moiety, on the other hand, are much less responsive to the 
exchange of the axial ligand. The replacement of chloride by azide results in changes $<5 \mathrm{~cm}^{-1}$ in both bands. Rather small shifts are also observed upon generation of the coordinated triazolate ligand in the course of the cycloaddition reaction and seem to depend on the further substitution pattern on the newly generated five-membered ring, with the antisymmetric stretch somewhat more responsive than the symmetric one. Replacement of the methyl ester group in the triazolates $\mathbf{5}$ and $\mathbf{1 1}$ by trifluoromethyl in $\mathbf{6}$ and $\mathbf{1 2}$ leads to somewhat larger shifts in the band positions but generally, these do not exceed about $20 \mathrm{~cm}^{-1}$.

The assignment of the ${ }^{1} \mathrm{H}$ NMR spectra is complicated by the presence of a mixture of exo and endo conformers due to two alternative orientations of the allyl group relative to the bpy ligand, with some of the signals overlapping. ${ }^{42-45}$ In addition, the triazolate ligand can assume two different coordination modes (N1 vs. N2) when carrying identical substituents in 4and 5-position, as in $\mathbf{5}$ and $\mathbf{1 1}$, while three different isomers are possible for the unsymmetrically substituted compounds 6 and 12. However, the trifluoromethyl group in alkyne $\mathbf{4}$ as well as triazolate complexes $\mathbf{6}$ and $\mathbf{1 2}$ is a sensitive and diagnostic marker for ${ }^{19} \mathrm{~F}$ NMR spectroscopy. In 4,4,4-trifluoro-2-butynoic acid ethyl ester (4), its resonance is observed at $-50.9 \mathrm{ppm}$ while in the resulting triazolate complexes, it is shifted upfield by about -6 to $-8 \mathrm{ppm}$ to approx. $-58 \mathrm{ppm}$. In the case of molybdenum compound 6 , three signals are observed in that range with an intensity ratio of $80: 14: 6$, while the tungsten analogue 12 shows only two species in 88:12 ratio. However, since the differences in chemical shift are very small, it is not possible to assign the different species to either exo/endo isomers or the different coordination modes of the triazolate ligand.

Both azide complexes $\mathbf{2}$ and $\mathbf{1 0}$ as well as the four triazolate compounds 5, 6, 11, and 12 were characterized by single-crystal $\mathrm{X}$-ray structure analysis with the molecular structures shown in Fig. 1 and 2 as well as Fig. S1-S4. Relevant crystallographic parameters are listed in Table S1. The tungsten center in $\mathbf{1 0}$ is in a pseudooctahedral coordination environment with the bpy ligand and the cis-W(CO $)_{2}$ moiety in the central plane (Fig. 1). The azide group points away from the bpy with a W1-N1-N2 angle of $124.3(3)^{\circ}$ and is located on a mirror plane which intersects the two carbonyl ligands and the central bpy C6-C6a axis, with the two halves of the molecule symmetry-related to each other.

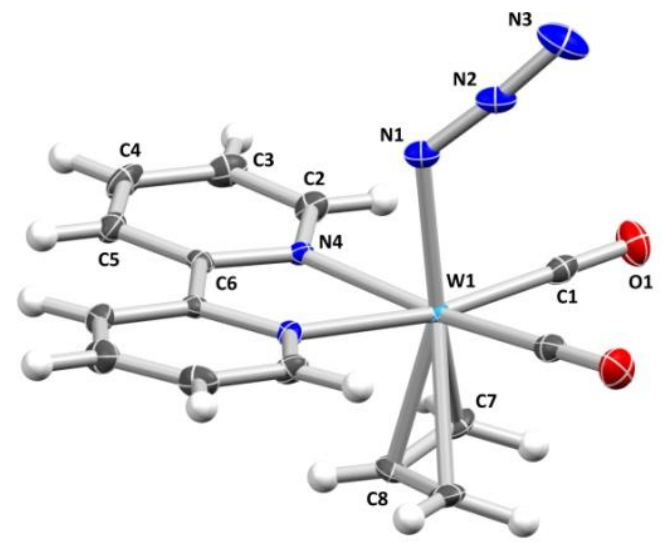

Fig. 1 Molecular structure of $\left[\mathrm{W}\left(\eta^{3}\right.\right.$-allyl $\left.)\left(\mathrm{N}_{3}\right)(\mathrm{bpy})(\mathrm{CO})_{2}\right] 10$ with thermal ellipsoids displayed at the $50 \%$ probability level. The asymmetric unit contains only one half of the molecule with the other one related to it by a mirror plane. Selected bond lengths [Å] and angles [ $\left.{ }^{\circ}\right]$ for 10: W1-C1 1.959(4), W1-N1 2.169(5), W1-N4 2.227(3), W1-(allyl centroid) 2.032(4), C1-O1 1.161(4), C7-C8 1.413(5), N1-N2 1.199(6), N2-N3 1.160(7), C1-W1-C1a 82.2(2), N4-W1-N4a 72.5(2), C1-W1-N4 102.0(2), N1-W1-(allyl centroid) $172.8(2), \mathrm{C} 7-\mathrm{C} 8-\mathrm{C7a}$ 114.5(5), W1-N1-N2 124.3(3), N1-N2-N3 175.4(5).

All other parameters, in particular long N1-N2 (1.199(6) $\AA$ ) and short N2-N3 (1.160(7) $\AA$ ) bond distances in the azide ligand, ${ }^{41}$ as well as C1-01 bond lengths of 1.161(4) $\AA$, are as expected. ${ }^{46}$ There are about a dozen tungsten azide complexes structurally characterized so far, ${ }^{47-55}$ but only one of them features a mixedligand carbonyl/azide coordination sphere related to $\mathbf{1 0}$. However, in $\left[\mathrm{W}\left(\eta^{3}\right.\right.$-allyl) $\left.\left(\mathrm{N}_{3}\right)(\mathrm{CO})_{2}(\mathrm{en})\right]$, the azide ligand is trans to one of the two carbonyl groups, ${ }^{53}$ while in 10 , it is trans to the allyl moiety. The metrical parameters of molybdenum azide complex $\mathbf{2}$ are essentially identical to those of the tungsten congener 10, with differences in bond lengths and angles below $0.01 \AA$ and $0.5^{\circ}$, respectively (Fig. S1). Most closely related structurally characterized compounds include $\left[\mathrm{Mo}\left(\eta^{3}\right.\right.$-allyl) $\left.\left(\mathrm{N}_{3}\right)(\mathrm{CO})_{2}(\mathrm{en})\right]{ }^{53}$ also isostructural to the tungsten complex mentioned above, two molybdenum dicarbonyl complexes with chelating diphosphane ligands of general formula $\left[\mathrm{Mo}\left(\eta^{3}\right.\right.$-allyl) $\left.\left(\mathrm{N}_{3}\right)(\mathrm{CO})_{2}\left(\mathrm{R}_{2} \mathrm{P}\left(\mathrm{CH}_{2}\right)_{n} \mathrm{PR} 2\right)\right]$, which vary in the linker length and substituent on the phosphorous center $(n=2, \mathrm{R}=$ $\mathrm{C}_{6} \mathrm{H}_{5}$ vs. $\left.n=1, \mathrm{R}=\mathrm{CH}_{3}\right),{ }^{22,56}$ and $\left[\mathrm{Mo}\left(\mathrm{N}_{3}\right)(\mathrm{NO})\left(\mathrm{bpm}^{\mathrm{CH} 3, \mathrm{CH} 3}\right)(\mathrm{CO})_{2}\right]$ with bpm $^{\mathrm{CH} 3, \mathrm{CH} 3}=$ bis(3,5-dimethyl-pyrazol-1-yl)methane. ${ }^{57}$ However, in all these compounds, the azide ligand is oriented trans to one of the diatomic ligands, either $\mathrm{CO}$ or NO, not the allyl.

All four triazolate complexes 5, 6, 11, and 12 obtained by iClick reaction with the electron-poor alkynes dimethyl acetylenedicarboxylate (DMAD, 3) or 4,4,4-trifluoro-2-butynoic acid ethyl ester (4) exhibit N2-coordination of the five-membered ring (Fig. 2 and Fig. S2-S4), similar to the other compounds prepared this way we have characterized so far. $^{19,28}$ Since the azide ligand is N1coordinated in $\mathbf{2}$ and 10, apparently a 1,2-shift has to take place during or after the cycloaddition reaction. The bond lengths and angles of all four triazolate complexes 5, 6, 11, and 12 are essentially identical, with deviations below $0.02 \AA$ and $0.3^{\circ}$, respectively. Apparently, the influence of the metal center (Mo vs. W) and substituent in the 4- and 5-position on the triazolate ring (methyl ester vs. trifluoromethyl/ethyl ester) on these parameters is negligible.

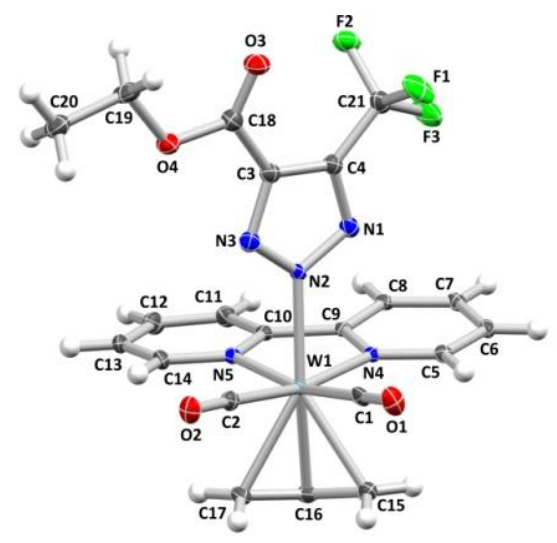


Fig. 2 Molecular structure of $\left[\mathrm{W}\left(\eta^{3}\right.\right.$-allyl)(triazolate $\left.\left.\mathrm{CF}^{\mathrm{C} 3, \mathrm{COOCH} 3}\right)(\mathrm{bpy})(\mathrm{CO})_{2}\right] 12$ with thermal ellipsoids displayed at the $50 \%$ probability level. Selected bond lengths $[\AA ̊]$ and angles [] for 12: W1-C1 1.957(2), W1-C2 1.965(2), W1-N2 2.206(2), W1-N4 2.213(2), W1-N5 2.224(2), W1-(allyl centroid) 2.031(2), C1-01 1.163(3), C2-O2 1.158(2), C15-C16 1.415(4), C16-C17 1.413(4), C1-W1-C2 78.0(1), N4-W1-N5 73.02(7), C1-W1-N4 105.22(9), C2-W1-N5 101.89(9), N2-W1-(allyl centroid) 176.27(7), C15-C16-C17 115.1(2), (N2-W1-C16)-(triazolate) 49.5(3).

The same is also true when comparing the azide vs. triazolate complexes, where differences of key parameters are below $0.02 \AA$ and $0.8^{\circ}$, respectively. One notable exception, however, is the orientation of the triazolate ring plane relative to the central $\mathrm{C}-\mathrm{C}$ axis of the bpy ligand. In the trifluoromethyl-substituted molybdenum compound 6 , these two are essentially parallel to each other and the triazolate assumes a staggered orientation relative to the two bipyridine $\mathrm{N}-$ metal-CO angles. In contrast, in the other three complexes $\mathbf{5}, \mathbf{1 1}$, and $\mathbf{1 2}$, the triazolate ring plane is significantly rotated around the $\mathrm{M}-\mathrm{N} 2$ axis relative to the central bpy $\mathrm{C}-\mathrm{C}$ vector and assumes a more or less eclipsed orientation relative to one of the $\mathrm{M}-\mathrm{CO}$ moieties. In the absence of any notable intramolecular interactions, the twist around the metal-triazolate $\mathrm{N} 2$ bond is apparently governed by subtle packing effects in the solid state. A detailed analysis of the energy barrier between the different conformations based on DFT calculations is presented below.

\section{${ }^{19} \mathrm{~F}$ NMR kinetic measurements}

The rate constant of the cycloaddition reaction is a very important parameter when this method is to be applied to bioconjugation, since it has to be faster than the biological process of interest. With 4,4,4-trifluoro-2-butynoic acid ethyl ester (4) as the alkyne component, the use of ${ }^{19} \mathrm{~F} N M R$ spectroscopy is a facile way to follow the course of the reaction, since there is a shift of about $-8 \mathrm{ppm}$ between the alkyne $\mathrm{CF}_{3}$ signal, ${ }^{28}$ which is observed at $-50.9 \mathrm{ppm}$ in DMSO$d_{6}$, and that of the trifluoromethyl group in the 4-position of the molybdenum- and tungsten-coordinated triazolates resulting from the cycloaddition, where the peaks are found at around $-58 \mathrm{ppm}$, with very little variance between the two metals.

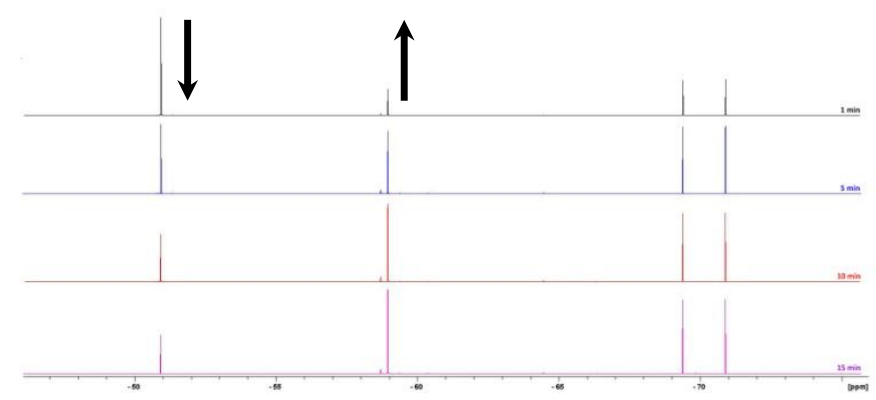

Fig. 3 Changes in the ${ }^{19} \mathrm{~F}$ NMR spectra $\left(470.59 \mathrm{MHz}\right.$, DMSO- $\left.d_{6}\right)$ of a mixture of tungsten azide complex 10 (35 mM), 4,4,4-trifluoro-2-butynoic acid ethyl ester (4, $35 \mathrm{mM}$ ) and ammonium hexafluorophoshate $(17 \mathrm{mM})$ at $27{ }^{\circ} \mathrm{C}$ over 1-15 min. Due to an experimental lag time no data could be obtained for the initial phase of the reaction $<1$ $\min$.

Thus, the tungsten azide complex 10 was dissolved in DMSO- $d_{6}$ and mixed with ammonium hexafluorophosphate serving as an internal standard. Then, alkyne 4 was added with the metal azide complex-to-alkyne ratio adjusted to $1: 1$. A series of ${ }^{19} \mathrm{~F}$ NMR spectra were recorded at $27{ }^{\circ} \mathrm{C}$ in $40-50 \mathrm{~s}$ intervals over $30 \mathrm{~min}$, with a time lag of about $1 \mathrm{~min}$ between the mixing and the first spectrum obtained due to experimental limitations. In addition to the signal of the alkyne at $-50.9 \mathrm{ppm}$ and the doublet of the hexafluorophosphate anion at -70.1 ppm with a ${ }^{1} J_{F, P}$ coupling of $711 \mathrm{~Hz}$, already the first spectrum recorded shows two additional signals at -58.7 and $-59.0 \mathrm{ppm}$ (Fig. 3 top). With increasing reaction time, these two signals assigned to the isomeric N1- and N2-coordinated triazolate complexes increased in intensity, while the alkyne $\mathrm{CF}_{3}$ peak at $-50.9 \mathrm{ppm}$ decreased continuously with similar time profiles (Fig. 4). In contrast, the intensity of the signal of the hexafluorophosphate additive remained constant during the whole time of the measurement.

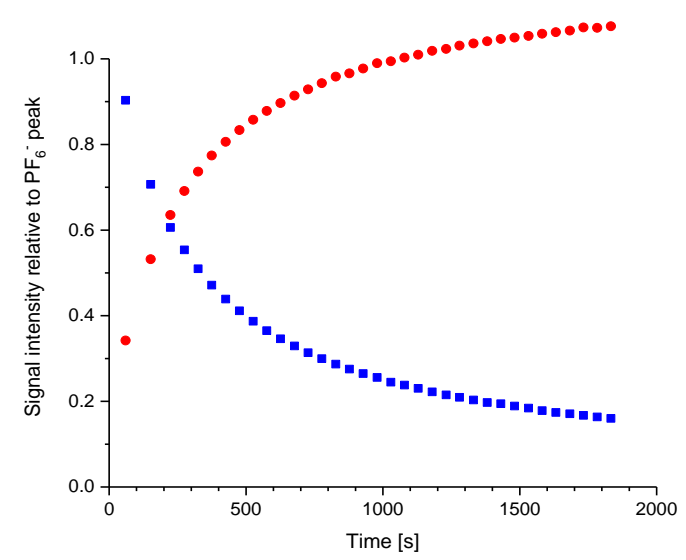

Fig. 4 Change of the ${ }^{19} \mathrm{~F}$ NMR peak intensity of the signals of the tungsten triazolate complex 12 at around -58 ppm (red circles) and of 4,4,4-trifluoro-2-butynoic acid ethyl ester (4) at $-50.9 \mathrm{ppm}$ (blue squares) with reaction time (1-30 $\mathrm{min}$ ) at $27^{\circ} \mathrm{C}$. Due to an experimental lag time no data could be obtained for the initial phase of the reaction $<1$ $\min$.

Assuming a second-order rate law and equal concentrations of azide complex and alkyne at the start of the reaction as well as negligible side-reactions, the kinetics can be modelled up to $50 \%$ conversion by eq. 1 .

$\frac{1}{\left.\text { alkyne }_{t=0}\right]}=\frac{1}{\left[\text { alkyne }_{t}\right]}+k_{2} t$

With an initial concentration of the alkyne of $35 \mathrm{mmol} \mathrm{L}^{-1}$, the second-order rate constant $k_{2}$ was determined by a linear fit from the slope of a plot of $1 /$ [alkyne al=0 ]-1/[alkyne $t$ ] over $t$ as $(7.3 \pm 0.1) \times 10^{-2} \mathrm{M}^{-1} \mathrm{~s}^{-1}$ at $27^{\circ} \mathrm{C}$. This is about one order of magnitude faster than the value of $k=7.6 \times 10^{-3} \mathrm{M}^{-1} \mathrm{~s}^{-1}$ reported by Veige and coworkers for the iClick reaction of $\left[\mathrm{Au}\left(\mathrm{N}_{3}\right)\left(\mathrm{PPh}_{3}\right)\right]$ with $\left[\mathrm{Au}\left(\mathrm{C} \equiv \mathrm{C}-\mathrm{C}_{6} \mathrm{H}_{5}\right)\left(\mathrm{PPh}_{3}\right)\right] .{ }^{18}$ The rate constant determined for the iClick reaction of the tungsten azide complex is comparable to those reported, for example, for the strain-promoted azide-alkyne cycloaddition (SPAAC) of firstgeneration compounds such as difluorocyclooctyne (DIFO), but about 1-2 orders of magnitude slower than values obtained for the latest reagents such as aza-dibenzocyclooctyne (DIBAC), biarylazacyclooctynone (BARAC), and 3,3,6,6- 
tetramethyl-thiacycloheptyne (TMTH). ${ }^{9}$ Other bioorthogonal coupling reactions such as the tetrazine ligation show even faster kinetics with rate constants up to $10^{4} \mathrm{M}^{-1} \mathrm{~s}^{-1} .{ }^{10}$ The same method was also attempted to study the kinetics of the iClick reaction of the related molybdenum azide complex $\mathbf{2}$ with alkyne 4. However, the reaction proceeded very fast at room temperature and after 2-3 $\mathrm{min}$, no signal for the trifluoromethyl-substituted alkyne was detectable at -50.9 ppm. This is a strong indication that the type of metal center (Mo vs. W) has a significant influence on the rate constant, but apparently the NMR spectroscopic method successfully applied to the tungsten complex has too long a time lag between mixing and recording of the first spectrum as well as too long delay times between the individual measurements to follow the process in the case of the molybdenum compound.

\section{Solution IR kinetic measurements}

Since the iClick reaction was too fast to be monitored by NMR in the case of the molybdenum complex $\mathbf{2}$ and comparison of the cycloaddition reactions of the azide complexes with DMAD (3) was not possible using this method, since it lacks the fluorine label as in 4,4,4-trifluoro-2-butynoic acid ethyl ester (4), solution IR measurements were explored as an alternative method to obtain the rate constants for the iClick reaction of 2 and $\mathbf{1 0}$ with the two alkynes $\mathbf{3}$ and $\mathbf{4}$, as previously used successfully to study the cycloaddition reaction of $\left[\mathrm{Rh}\left(\mathrm{Cp}^{*}\right)\left(\mathrm{N}_{3}\right)\left(\mathrm{bpy}^{\mathrm{R}, \mathrm{R}}\right)\right] \mathrm{CF}_{3} \mathrm{SO}_{3}$ with alkyne $4 .^{19}$ Thus, the azide complex (2 or 10,8 mM) dissolved in dimethylsulfoxide was mixed with alkyne (3 or $4,40 \mathrm{mM}$ ) and then quickly transferred to a liquid IR cell, with a maximum time lag of about 1.5 min between mixing and start of the measurement. Measurements in the $1800-2100 \mathrm{~cm}^{-1}$ spectral range were carried out in $20 \mathrm{~s}$ intervals until the azide band at 2050-2060 $\mathrm{cm}^{-1}$ had completely disappeared. No spectral overlap was observed in that region with bands assigned to alkynes $\mathbf{3}$ and $\mathbf{4}$ (Fig. S5). In contrast to the azide band, the two signals due to the antisymmetric and symmetric $\mathrm{C} \equiv \mathrm{O}$ stretching vibrations between 1835 and $1935 \mathrm{~cm}^{-1}$ remained essentially unchanged during the course of the reaction (Fig. 5 and Fig. S6). A minor intermediate decrease in the intensity of the symmetric $\mathrm{C} \equiv \mathrm{O}$ band might be due to subtle differences in the extinction coefficients of this vibration in the azide vs. triazolate species. Significant differences in the time required for the reaction to reach completion are clearly evident in a plot of the intensity of the azide stretch vs. reaction time (Fig. 6). The reaction of the azide complexes $\mathbf{2}$ and $\mathbf{1 0}$ with 4,4,4-trifluoro-2-butynoic acid ethyl ester (4) is faster than that with DMAD (3) regardless of the metal complex, while the molybdenum azide complex reacts faster than the tungsten analogue irrespective of the alkyne. The data was fit to a monoexponential decay according to eq. 2 with a correlation coefficient of better than 0.9990 for all four reactions and gave pseudo-first order rate constants $k$ in the range of 0.4 to $6.5 \times 10^{-3} \mathrm{~s}^{-1}$ (Table 2).

$A\left(\mathrm{~N}_{3}\right)=A_{1} \cdot e^{-\frac{t}{t_{1}}}+y_{0}$
The molybdenum azide complex 2 reacts about 3-4 times faster than the tungsten analogue $\mathbf{1 0}$, while the cycloaddition reaction with 4,4,4-trifluoro-2-butynoic acid ethyl ester (4) is 4-7 times more rapid than the one with DMAD (3).
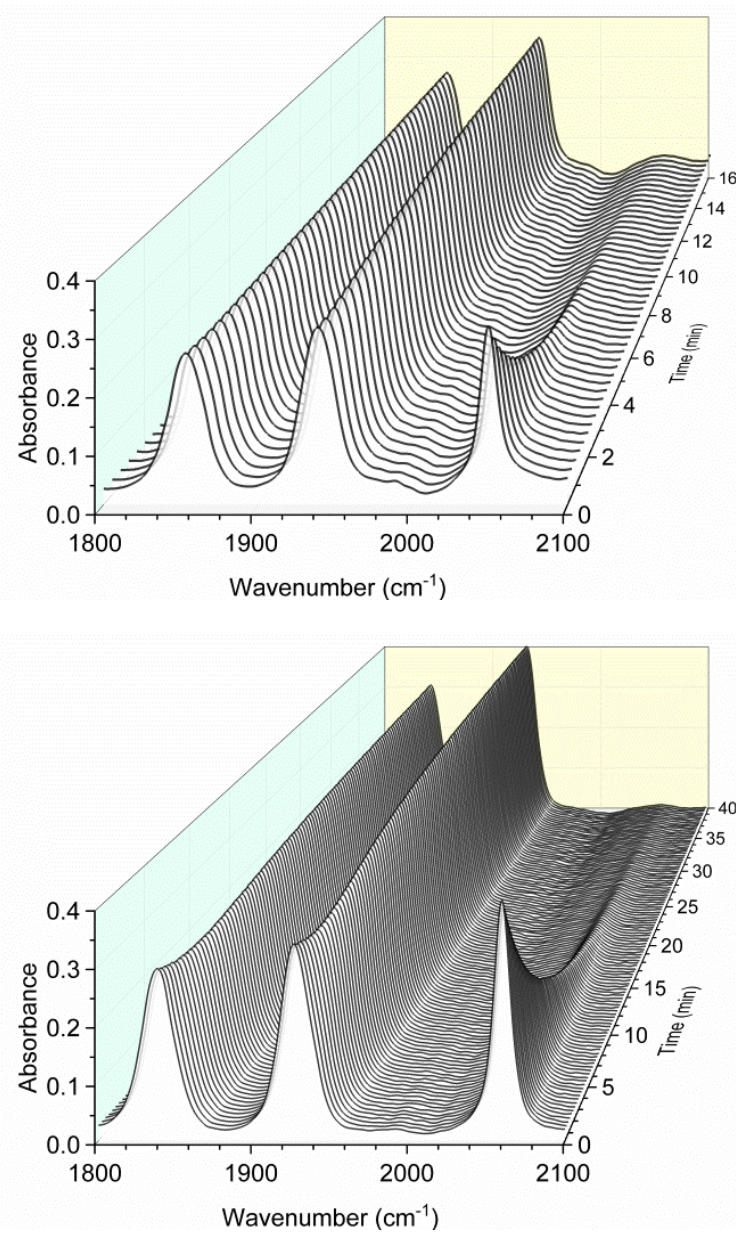

Fig. 5 Changes in the $1800-2100 \mathrm{~cm}^{-1}$ spectral range of the IR spectrum of a mixture of $\mathbf{2}$ (top) or $\mathbf{1 0}$ (bottom) in dimethylsulfoxide $(8 \mathrm{mM}$ ) upon reaction with alkyne $\mathbf{4}$ (40 $\mathrm{mM}$ ) at $26^{\circ} \mathrm{C}$ in $20 \mathrm{~s}$ intervals for up to $40 \mathrm{~min}$.

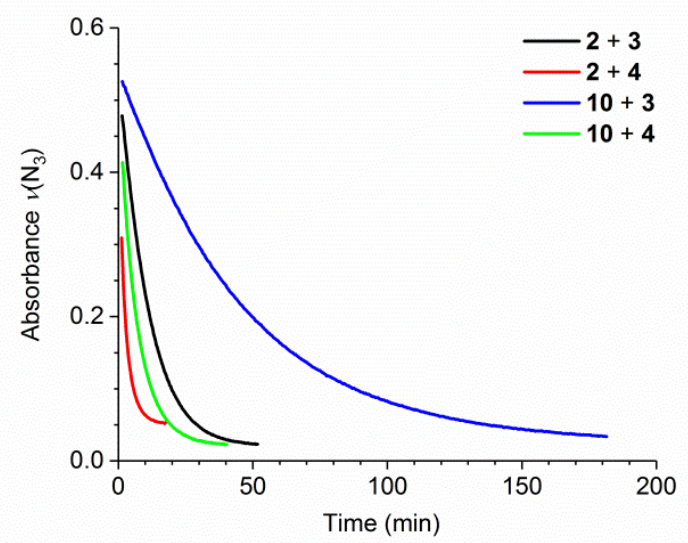

Fig. 6 Changes in the intensity of the azide stretch of complexes 2 or $10(8 \mathrm{mM})$ upon reaction with alkynes 3 or $4(40 \mathrm{mM})$ in dimethylsulfoxide at $26^{\circ} \mathrm{C}$ for up to $180 \mathrm{~min}$. 
While the latter trend corresponds well with an increase in the rate constant with increasing electron density on one of the organic reactants, as observed previously for $\left[\mathrm{Rh}\left(\mathrm{Cp}^{*}\right)\left(\mathrm{N}_{3}\right)\left(\mathrm{bpy}^{\mathrm{R}, \mathrm{R}}\right)\right] \mathrm{CF}_{3} \mathrm{SO}_{3}{ }^{19}$ and reflected in the Hammett constants for $\mathrm{COOCH}_{3}$ vs. $\mathrm{CF}_{3}$ of 0.45 and 0.54 , respectively, ${ }^{58}$ the influence of the metal on the speed of the iclick reaction has not been studied so far and thus there is no data available for comparison. Unfortunately, the isostructural chromium azide complex was not accessible for comparison due to low stability of the starting materials.

Table 2 Pseudo-first order rate constants $k$ and half-lives $t_{1 / 2}$ for the cycloaddition reaction of azide complexes $\mathbf{2}$ or $\mathbf{1 0}$ with alkynes $\mathbf{3}$ or $\mathbf{4}$ in dimethylsulfoxide at $26^{\circ} \mathrm{C}$.

\begin{tabular}{ccccc}
\hline reactants & metal & alkyne & $k\left(\right.$ in $\left.10^{-3} \mathrm{~s}^{-1}\right)$ & $t_{1 / 2}$ (in s) \\
\hline $\mathbf{1 0}+\mathbf{3}$ & $\mathrm{W}$ & DMAD & $0.4 \pm 0.1$ & 1896 \\
$\mathbf{2}+\mathbf{3}$ & Mo & DMAD & $1.6 \pm 0.1$ & 448 \\
$\mathbf{1 0 + 4}$ & $\mathrm{W}$ & $\mathrm{F}_{3} \mathrm{C}-\mathrm{C} \equiv \mathrm{C}$-COOEt & $2.5 \pm 0.1$ & 274 \\
$\mathbf{2}+\mathbf{4}$ & Mo & $\mathrm{F}_{3} \mathrm{C}-\mathrm{C} \equiv \mathrm{C}$-COOEt & $6.5 \pm 0.1$ & 107 \\
\hline
\end{tabular}

Overall, however, the rate constants compare well with those of the abovementioned RhCp ${ }^{*}$ complexes $^{19}$ of 2.4 to $3.8 \times 10^{-3}$ $\mathrm{s}^{-1}$ and the ones reported by Veige et al. for the iClick reaction of $\left[\mathrm{Au}\left(\mathrm{N}_{3}\right)\left(\mathrm{PPh}_{3}\right)\right]$ with gold(I) phenylacetylides. ${ }^{59}$

\section{DFT calculations}

In order to obtain some insight into the energy hypersurface of the triazolate complexes, in particular with regard to the orientation of the allyl ligand (exo vs. endo), the coordination mode of the five-membered ring (N1 vs. N2), and the rotation of the triazolate around the metal- $\mathrm{N}$ axis, DFT calculations were carried out on one of the molybdenum complexes serving as a model system, using the BP86 functional, a TZVP basis set, and the COSMO solvation model using dimethylsulfoxide, as also employed in the NMR studies.

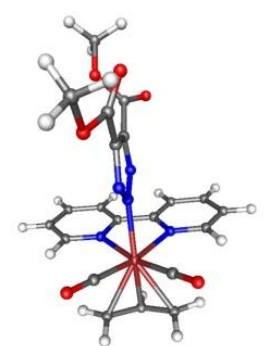

0.0

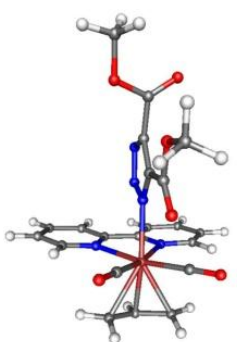

6.9

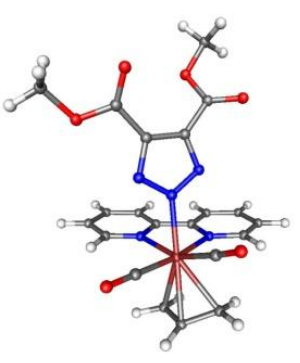

6.2
Fig. 7 DFT-optimized structures (ORCA 2.8, BP86, RI, TZVP, COSMO in DMSO) of (left) the N2-coordinated endo isomer, (center) the N1-coordinated exo isomer, and (right) the N2-coordinated endo isomer of $\mathbf{5}$. Relative energies are reported in $\mathrm{kcal} \mathrm{mol}^{-1}$.

A full geometry optimization without constraints showed the $\mathrm{N} 2$-coordinated endo isomer of $\mathbf{5}$ to be $6.2 \mathrm{kcal} \mathrm{mol}^{-1}$ more stable than the exo one. Retaining the endo orientation of the allyl ligand, the corresponding N1 species was destabilized by $6.9 \mathrm{kcal} \mathrm{mol}^{-1}$ relative to the reference isomer (Fig. 7), which is also confirmed by other recent structural studies. ${ }^{60,61}$ Thus, the triazolate coordination mode and allyl ligand orientation observed in the X-ray structure of $\mathbf{5}$ is indeed the most stable of the three isomers studied. However, due to the many degrees of freedom, the additional effect of rotation of the methyl ester groups around the triazolate- $\mathrm{C} 4 / \mathrm{C} 5-\mathrm{COOCH}_{3}$ axis and variation of the $\mathrm{H}_{3} \mathrm{C}-\mathrm{O}-\mathrm{C}=\mathrm{O}$ torsion angle was not further investigated.

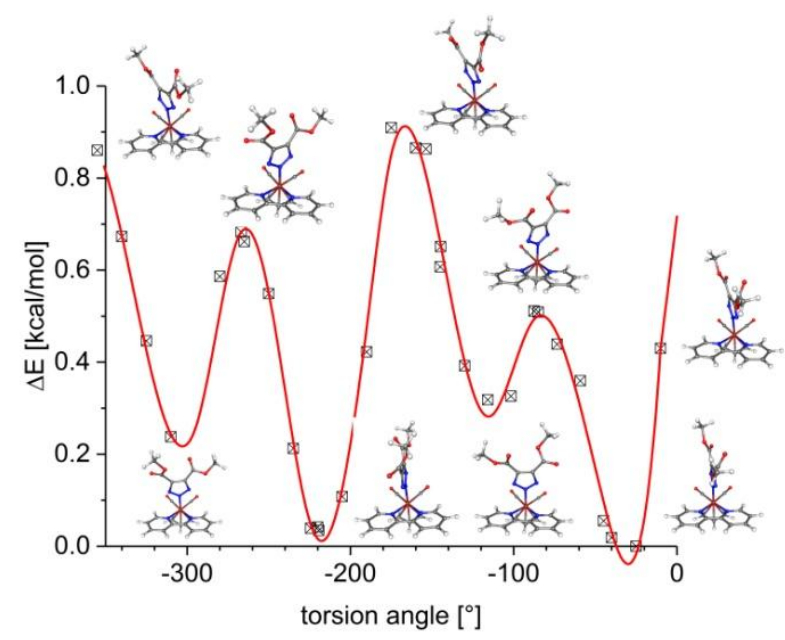

Fig. 8 Conformational energy diagram of $\mathbf{5}$ for variation of the torsion angle between the triazolate mean plane and the bpy C2-C2' axis calculated with DFT (ORCA 2.8, BP86, $\mathrm{RI}, \mathrm{TZVP}, \mathrm{COSMO}$ in DMSO).

A relaxed surface scan of the N2-coordinated endo isomer with rotation of the triazolate around the Mo-N2 bond in $15^{\circ}$ steps showed the "parallel" orientation of the triazolate mean plane and the central bpy $\mathrm{C}-\mathrm{C}$ axis as well as the "perpendicular" conformation with the triazolate at $90^{\circ}$ relative to the bipyridine central axis to be minimum structures, with the latter slightly lower in energy by about 0.2 $\mathrm{kcal} \mathrm{mol}^{-1}$, while the eclipsed orientations are transition states between the four minimum structures (Fig. 8). However, with a maximum difference in energy of only $0.9 \mathrm{kcal} \mathrm{mol}^{-1}$, the barrier is extremely low, even less than the $1.3 \mathrm{kcal} \mathrm{mol}^{-1}$ determined for a $\left[\mathrm{Mn}\right.$ (triazolate)(bpy) $\left.(\mathrm{CO})_{3}\right]$ derivative in a previous study ${ }^{28}$ and only about one-third of the energy difference between the staggered and eclipsed conformations of ethane of about $2.9 \mathrm{kcal} \mathrm{mol}^{-1}$. $^{62}$ In the case of the unsymmetrically substituted molybdenum complex $\mathbf{6}$, a different energy profile was observed (Fig. S7), since the "perpendicular" conformer with the trifluoromethyl group pointing towards the bpy ring is significantly more stable than the one turned by $180^{\circ}$, with the ethyl ester group towards the bipyridine. Furthermore, the eclipse conformations now also become inequivalent. Still, even in this case, the maximum barrier height does not exceed $1.3 \mathrm{kcal} \mathrm{mol}^{-1}$. Thus, the triazolate ligand can rotate freely around the Mo-N2 axis at ambient temperature and there is only a very minor influence of the substituents in 4- and 5-position on the barrier height.

\section{Conclusions}

Two group $\mathrm{VI}$ azide complexes of the general formula $\left[\mathrm{M}\left(\eta^{3}-\right.\right.$ allyl) $\left(\mathrm{N}_{3}\right)(\mathrm{bpy})(\mathrm{CO})_{2}$ ] with $\mathrm{M}=\mathrm{Mo}, \mathrm{W}$ were prepared and fully characterized, including X-ray structure analysis, which revealed little differences between the two compounds. While 
the molybdenum(II) complex was accessible in two steps, the one-pot reaction of $\left[\mathrm{Mo}(\mathrm{CO})_{6}\right]$ with allyl chloride and 2,2'bipyridine followed by exchange of the chloride to the azide ligand, the tungsten(II) analogue required a different synthetic strategy due to significantly slower ligand exchange. Initially, two of the CO ligands in tungsten(0) hexacarbonyl were removed with TMAO and then replaced by bpy. This was followed by exchange of a third carbonyl ligand by pyridine in refluxing xylene. The resulting $\left[\mathrm{W}(\mathrm{bpy})(\mathrm{CO})_{3}(\mathrm{py})\right]$ was then employed in a further reaction sequence similar to the one employed for the molybdenum complex. The two azide complexes underwent smooth [3+2] cycloaddition iClick reactions with electron-poor alkynes such as dimethyl acetylenedicarboxylate (DMAD) and 4,4,4-trifluoro-2-butynoic acid ethyl ester, while phenyltrifluoromethylacetylene did not react. However, at present it is unclear whether this is due to electronic or steric factors. The four triazolate compounds obtained were characterized by $\mathrm{X}$-ray structure analysis, which revealed the five-membered ring to be coordinated to the metal center via the N2 nitrogen atom in all cases. In contrast, ${ }^{19} \mathrm{~F}$ NMR spectra of the trifluoromethyl-substituted products gave hints of a mixture of the N1- and N2-coordinated species in solution, although the assignment is complicated by the additional exo/endo isomerism of the ally ligand. Still, the method allowed facile study of the reaction kinetics in the case of the tungsten azide complex, which gave a second-order rate constant of $(7.3 \pm 0.1) \times 10^{-2} \mathrm{M}^{-1} \mathrm{~s}^{-1}$, comparable to that observed for some first-generation cyclic alkynes used in the strain-promoted azide-alkyne cycloaddtion (SPAAC). The analogous molybdenum complex reacted too fast for study with NMR methods and attempts to also prepare the similar chromium compound did not succeed due to very low stability of the precursors. On the other hand, solution IR measurements allowed the determination of pseudo-first order rate constants for all four combinations of metal-azide complexes and alkynes, which were found to be in a range of 0.4 to $6.5 \times 10^{-3} \mathrm{~s}^{-1}$ and increased in the order of $\mathrm{Mo}>\mathrm{W}$ and $\mathrm{F}_{3} \mathrm{C}-\mathrm{C} \equiv \mathrm{C}$-COOEt $>$ DMAD. Thus, for the first time, there has been a systematic evaluation of the effect of the metal center on the kinetics of the iclick cycloaddition reaction of metalcoordinated azides with electron-poor alkynes. In otherwise isostructural and isoelectronic compounds, the $4 \mathrm{~d}$ complex was consistently found to react faster than the $5 d$ analog. This demonstrates that the kinetics of the iclick reaction of metalcoordinated small ligands can be tuned not only by variation of electron-withdrawing vs. electron-donating groups in the periphery of the alkyne ${ }^{18}$ or the coligands, ${ }^{19}$ but also by exchange of the metal itself. Thus, it is expected that this reaction can be extended to additional transition metal azide complexes and such experiments are currently under way. In contrast, the choice of the alkyne seems to be much more limited, as demonstrated by the failure of phenyltrifluoromethylacetylene to give any cycloaddition product, even at elevated temperatures. More work will be required to dissect the electronic and steric factors which govern the reactivity of this component and establish a general building block approach to metal-triazolate complexes. ${ }^{13}$

\section{Experimental section}

\section{Materials and instruments}

Reactions were carried out in oven-dried Schlenk glassware under an atmosphere of pure argon or dinitrogen and reaction vessels were protected from light by wrapping with aluminium foil if necessary, in particular for the carbonyl complexes. Molybdenum and tungsten hexacarbonyl were supplied by Strem. All other chemicals were purchased from commercial sources and used as received. 4,4,4-Trifluoro-2-butynoic acid ethyl ester and dimethyl-7oxa-bicyclo[2.2.1] hepta-2,5-diene-2,3-dicarboxylate were prepared by published procedures. ${ }^{33,35}$

IR spectra of pure solid samples were obtained using a Nicolet 380 FT-IR spectrometer fitted with a smart iTR ATR accessory. The solution IR kinetic measurements were carried out with a JASCO FTIR 4100 instrument in an Omni-Cell 31800 liquid sample holder composed of calcium fluoride windows (width: $4 \mathrm{~mm}$ ) with a Teflon $\operatorname{spacer}(d=200 \mu \mathrm{m})$.

NMR spectra were recorded on Bruker Avance $200\left({ }^{1} \mathrm{H}: 200.13\right.$ $\left.\mathrm{MHz},{ }^{13} \mathrm{C}: 50.32 \mathrm{MHz},{ }^{19} \mathrm{~F}: 188.12 \mathrm{MHz}\right)$, Avance $400\left({ }^{1} \mathrm{H}: 400.40\right.$ $\left.\mathrm{Mhz},{ }^{13} \mathrm{C}: 100.68 \mathrm{MHz},{ }^{19} \mathrm{~F}: 376.76 \mathrm{MHz}\right)$, and Avance $500\left({ }^{1} \mathrm{H}\right.$ : $500.13 \mathrm{MHz},{ }^{13} \mathrm{C}: 125.76 \mathrm{MHz},{ }^{19} \mathrm{~F}: 470.59 \mathrm{MHz},{ }^{31} \mathrm{P}: 202.46 \mathrm{~Hz}$ ) spectrometers. In the case of the ${ }^{1} \mathrm{H}$ and ${ }^{13} \mathrm{C} N M R$, they were referenced relative to the residual signal of the solvent ${ }^{63}$ while they are given relative to $\mathrm{CFCl}_{3}$ in the case of the ${ }^{19} \mathrm{~F} \mathrm{NMR}$. Peak multiplicities are marked as singlet (s), doublet (d), doublet of doublet (dd), doublet of doublet of doublet (ddd), doublet of triplet $(\mathrm{dt})$, triplet $(\mathrm{t})$, and multiplet $(\mathrm{m})$, respectively and coupling constants $J$ are given in Hertz $(\mathrm{Hz})$. The elemental composition of the compounds was determined with an Elementar Vario MICRO cube $\mathrm{CHN}$ analyzer. Addition of $\mathrm{V}_{2} \mathrm{O}_{5}$ was usually required to obtain proper results in the case of the metal complexes.

\section{Synthesis}

[Mo( $\eta^{3}$-allyl)Cl(bpy)(CO) $)_{2}$ (1). To a degassed mixture of anhydrous tetrahydrofuran $(20 \mathrm{~mL})$ and allyl chloride $(1.6 \mathrm{~mL}$, $1.5 \mathrm{~g}, 19.7 \mathrm{mmol}$ ), molybdenum hexacarbonyl (500 mg, 1.9 mmol) and 2,2'-bipyridine (265 $\mathrm{mg}, 1.7 \mathrm{mmol}$ ) were added under a dinitrogen atmosphere and the mixture heated to reflux for $18 \mathrm{~h}$. The resulting red precipitate was filtered from the purple solution, washed with $n$-hexane $(4 \times 25 \mathrm{~mL})$ and dried under vacuum to give a bright red solid. Yield: 76\% (486 $\mathrm{mg}, 1.3 \mathrm{mmol}$ ). Elemental analysis (\%): calc. $\mathrm{C}_{15} \mathrm{H}_{13} \mathrm{ClMoN}_{2} \mathrm{O}_{2}$ : C 46.84, H 3.41, N 7.28, found: C 46.55, H 3.48, N 7.15; IR (ATR,

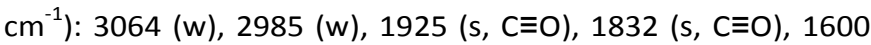
$(\mathrm{m}), 1469(\mathrm{~m}), 1442(\mathrm{~m}), 1310(\mathrm{w}), 1152(\mathrm{w}), 1024(\mathrm{w}), 769$ $(\mathrm{m}) ;{ }^{1} \mathrm{H}$ NMR (DMSO- $d_{6}, 200.13 \mathrm{MHz}, \mathrm{ppm}$ ): $\delta$ major isomer: $8.77\left(\mathrm{~d}, 2 \mathrm{H},{ }^{3} \mathrm{~J}_{\mathrm{H} 6, \mathrm{H} 5}=4.9 \mathrm{~Hz}, \mathrm{bpy}-\mathrm{H} 6\right), 8.56\left(\mathrm{~d}, 2 \mathrm{H},{ }^{3} \mathrm{~J}_{\mathrm{H} 3, \mathrm{H} 4}=8.0\right.$ $\mathrm{Hz}$, bpy-H3), $8.17\left(\mathrm{dt}, 2 \mathrm{H},{ }^{3} J_{\mathrm{H} 4, \mathrm{H} 3 / \mathrm{H} 5}=8.0 \mathrm{~Hz},{ }^{4} J_{\mathrm{H} 4, \mathrm{H} 6}=1.0 \mathrm{~Hz}\right.$, bpy-H4), $7.63\left(\mathrm{t}, 2 \mathrm{H},{ }^{3} \mathrm{~J}_{\mathrm{H} 5-\mathrm{H} 4 / \mathrm{H} 6}=6.3 \mathrm{~Hz}\right.$, bpy-H5), 3.12-3.27 (m, $1 \mathrm{H}$, allyl- $\left.\mathrm{H}_{\text {meso }}\right), 3.07\left(\mathrm{~d}, 2 \mathrm{H},{ }^{3} J_{\text {Hsyn, Hmeso }}=6.2 \mathrm{~Hz}\right.$, allyl- $\left.\mathrm{H}_{\text {syn }}\right), 1.23$ $\left(\mathrm{d}, 2 \mathrm{H},{ }^{3} J_{\text {Hanti,Hmeso }}=8.7 \mathrm{~Hz}\right.$, allyl- $\left.\mathrm{H}_{\text {anti }}\right)$; minor isomer: $9.06(\mathrm{~d}$, $\left.2 \mathrm{H},{ }^{3} \mathrm{~J}_{\mathrm{H} 6, \mathrm{H} 5}=3.8 \mathrm{~Hz}, \mathrm{bpy}-\mathrm{H} 6\right), 8.65\left(\mathrm{~d}, 2 \mathrm{H},{ }^{3} \mathrm{~J}_{\mathrm{H} 3, \mathrm{H} 4}=8.3 \mathrm{~Hz}\right.$, bpy$\mathrm{H} 3), 8.26\left(\mathrm{~d}, 2 \mathrm{H},{ }^{3} J_{\mathrm{H} 4, \mathrm{H} 3}=8.3 \mathrm{~Hz}, \mathrm{bpy}-\mathrm{H} 4\right), 7.79\left(\mathrm{t}, 2 \mathrm{H},{ }^{3} \mathrm{~J}_{\mathrm{H} 5}{ }^{-} \mathrm{H} 4 / \mathrm{H} 6\right.$ $=6.3 \mathrm{~Hz}$, bpy-H5), 3.79-3.91 (m, $1 \mathrm{H}$, allyl- $\left.\mathrm{H}_{\text {meso }}\right), 3.51(\mathrm{~d}, 2 \mathrm{H}$, ${ }^{3} J_{\text {Hsyn,Hmeso }}=6.2 \mathrm{~Hz}$, allyl- $\left.\mathrm{H}_{\text {syn }}\right), 1.36\left(\mathrm{~d}, 2 \mathrm{H},{ }^{3} J_{\text {Hanti,Hmeso }}=9.4 \mathrm{~Hz}\right.$, 
allyl- $\mathrm{H}_{\text {anti }}$ ); ${ }^{13} \mathrm{C}$ NMR (DMSO- $d_{6}, 50.32 \mathrm{MHz}, \mathrm{ppm}$ ): $\delta$ major isomer: 227.13 ( $\mathrm{C} \equiv \mathrm{O}), 153.30$ (bpy-C2), 151.85 (bpy-C6), 139.18 (bpy-C4), 126.27 (bpy-C5), 122.98 (bpy-C3), $70.76(\mathrm{CH}$ allyl), $54.00\left(\mathrm{CH}_{2}\right.$-allyl); minor isomer: the signal intensity was below the detection limit.

[Mo $\left(\eta^{3}\right.$-allyl)( $\left.\left.\mathbf{N}_{3}\right)(\mathrm{bpy})(\mathrm{CO})_{2}\right]$ (2). To a suspension of $\left[\mathrm{Mo}\left(\eta^{3}-\right.\right.$ allyl)Cl(bpy) $\left.(\mathrm{CO})_{2}\right] \quad(1,450 \mathrm{mg}, 1.2 \mathrm{mmol})$ in degassed acetonitrile $(50 \mathrm{~mL})$ was added silver triflate $(301 \mathrm{mg}, 1.2$ $\mathrm{mmol})$. A white precipitate appeared and the resulting suspension was stirred for $2 \mathrm{~h}$ under a dinitrogen atmosphere under exclusion of light. The clear red solution was then transferred with a teflon cannula into another flask containing sodium azide (152 $\mathrm{mg}, 2.3 \mathrm{mmol}$ ) and stirred at room temperature for $19 \mathrm{~h}$ while protected from light. The resulting red precipitate was filtered off, first washed with plenty of water to remove excess sodium azide, and then with ethanol $(2 \times 5 \mathrm{~mL})$ followed by diethylether $(2 \times 10 \mathrm{~mL})$ and subsequently dried under vacuum to give the product as a bright red solid. Yield: $58 \%$ (258 mg, $0.7 \mathrm{mmol})$. Elemental analysis (\%): calc. $\mathrm{C}_{15} \mathrm{H}_{13} \mathrm{MoN}_{5} \mathrm{O}_{2}$ : C 46.05, H 3.35, N 17.90, found: C 44.99, H 3.38, N 18.04; IR (ATR, cm ${ }^{-1}$ ): 3083 (w), 2036 (s, azide), 1928 $(\mathrm{s}, \mathrm{C} \equiv 0), 1836(\mathrm{~s}, \mathrm{C} \equiv \mathrm{O}), 1600(\mathrm{~m}), 1469(\mathrm{~m}), 1440(\mathrm{~m}), 1311$ $(\mathrm{w}), 1172(\mathrm{w}), 757(\mathrm{~m}), 733(\mathrm{w}) ;{ }^{1} \mathrm{H}$ NMR (DMSO-d $6,200.13$ $\mathrm{MHz}, \mathrm{ppm}$ ): $\delta$ major isomer: $8.80\left(\mathrm{dd}, 2 \mathrm{H},{ }^{3} J_{\mathrm{H} 6, \mathrm{H} 5}=5.3 \mathrm{~Hz},{ }^{4} J_{\mathrm{H} 6, \mathrm{H} 4}\right.$ $=0.9 \mathrm{~Hz}$, bpy-H6), $8.57\left(\mathrm{~d}, 2 \mathrm{H},{ }^{3} J_{\mathrm{H} 3, \mathrm{H} 4}=8.2 \mathrm{~Hz}, \mathrm{bpy}-\mathrm{H} 3\right), 8.20$ $\left(\mathrm{dt}, 2 \mathrm{H},{ }^{3} J_{\mathrm{H} 4, \mathrm{H} 3 / \mathrm{H} 5}=7.9 \mathrm{~Hz},{ }^{4} J_{\mathrm{H} 4, \mathrm{H} 6}=1.2 \mathrm{~Hz}, \mathrm{bpy}-\mathrm{H} 4\right), 7.66$ (ddd, $2 \mathrm{H},{ }^{3} J_{\mathrm{H} 5, \mathrm{H} 4}=7.9 \mathrm{~Hz},{ }^{3} J_{\mathrm{H} 5, \mathrm{H} 6}=5.4 \mathrm{~Hz},{ }^{4} J_{\mathrm{H} 5, \mathrm{H} 3}=1.1 \mathrm{~Hz}$, bpy-H5), $3.18-3.23\left(\mathrm{~m}, 1 \mathrm{H}\right.$, allyl- $\left.\mathrm{H}_{\text {meso }}\right), 3.14\left(\mathrm{~d}, 2 \mathrm{H},{ }^{3} \mathrm{~J}_{\text {Hsyn,Hmeso }}=6.7 \mathrm{~Hz}\right.$, allyl- $\left.\mathrm{H}_{\text {syn }}\right), 1.29\left(\mathrm{~d}, 2 \mathrm{H},{ }^{3} J_{\text {Hanti,Hmeso }}=8.9 \mathrm{~Hz}\right.$, allyl- $\left.\mathrm{H}_{\text {anti }}\right)$; minor isomer: $9.05(\mathrm{~m}, 2 \mathrm{H}$, bpy-H6), $8.69(\mathrm{~m}, 2 \mathrm{H}$, bpy-H3), $8.26(\mathrm{~m}$, $2 \mathrm{H}$, bpy-H4), $7.83(\mathrm{~m}, 2 \mathrm{H}$, bpy-H5), 3.71-3.89 (m, $1 \mathrm{H}$, allyl$\left.\mathrm{H}_{\text {meso }}\right), 3.20\left(\mathrm{~d}, 2 \mathrm{H},{ }^{3} \mathrm{~J}_{\text {Hsyn,Hmeso }}=8.1 \mathrm{~Hz}\right.$, allyl- $\left.\mathrm{H}_{\text {syn }}\right), 1.36(\mathrm{~d}, 2 \mathrm{H}$, ${ }^{3} J_{\text {Hanti,Hmeso }}=9.0 \mathrm{~Hz}$, allyl- $\left.\mathrm{H}_{\text {anti }}\right) ;{ }^{13} \mathrm{C}$ NMR (DMSO- $d_{6}, 50.32 \mathrm{MHz}$, ppm): $\delta$ major isomer: 227.20 (C三O), 153.27 (bpy-C2), 151.85 (bpy-C6), 139.48 (bpy-C4), 126.56 (bpy-C5), 123.04 (bpy-C3), 72.54 ( $\mathrm{CH}$-allyl), $55.78\left(\mathrm{CH}_{2}\right.$-allyl); minor isomer: the signal intensity was below the detection limit.

[Mo( $\eta^{3}$-allyl)(triazolate $\left.\left.{ }^{\mathrm{COOCH} 3, \mathrm{COOCH}_{3}}\right)(\mathrm{bpy})(\mathrm{CO})_{2}\right](5)$. Method A: $\left[\mathrm{Mo}\left(\eta^{3}\right.\right.$-allyl) $\left.\left(\mathrm{N}_{3}\right)(\mathrm{bpy})(\mathrm{CO})_{2}\right] \quad(2,44 \mathrm{mg}, 0.11 \mathrm{mmol})$ and dimethyl acetylenedicarboxylate (DMAD, $17 \mu \mathrm{L}, 20 \mathrm{mg}, 0.14$ $\mathrm{mmol})$ were dissolved in dichloromethane $(15 \mathrm{~mL})$ and stirred for $36 \mathrm{~h}$ at room temperature under exclusion of light. The resulting red solution was filtered through Celite. After concentration under vacuum, it was layered with $n$-hexane for crystallization. After $3 \mathrm{~d}$ in the dark, the red needles obtained were filtered off, washed with $n$-hexane and dried under vacuum. Yield: 45\% (28 mg, $0.05 \mathrm{mmol})$. Method B: [Mo $\left(\eta^{3}-\right.$ allyl) $\left(\mathrm{N}_{3}\right)\left(\right.$ bpy) $(\mathrm{CO})_{2}$ ] $(62 \mathrm{mg}, 0.16 \mathrm{mmol})$ and dimethyl-7-oxabicyclo[2.2.1] hepta-2,5-diene-2,3-dicarboxylate $(50 \mathrm{mg}, 0.24$ $\mathrm{mmol})$ were dissolved in dichloromethane $(20 \mathrm{~mL})$ and stirred for $48 \mathrm{~h}$ at room temperature under exclusion of light. The solvent was removed under vacuum and the red residue washed with ethyl acetate $(3 \times 5 \mathrm{~mL})$ followed by $n$-hexane $(3 \times 5$ $\mathrm{mL}$ ) and subsequently dried under vacuum to give a red powder. Yield: $75 \%$ (65 mg, $0.12 \mathrm{mmol}$ ). Experimental data is reported for the material obtained following procedure $\mathrm{B}$ : Elemental analysis (\%): calc. $\mathrm{C}_{21} \mathrm{H}_{19} \mathrm{MoN}_{5} \mathrm{O}_{6}$ : $\mathrm{C} 47.29, \mathrm{H} 3.59, \mathrm{~N}$
13.13, found: C 47.03, H 3.58, N 13.14; IR (ATR, $\mathrm{cm}^{-1}$ ): 3071

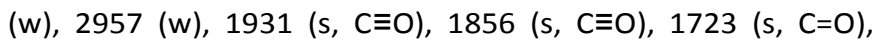
$1600(w), 1439(m), 1292(w), 1220(m), 1163(m), 1090(m)$, $764(\mathrm{~m}) ;{ }^{1} \mathrm{H}$ NMR (DMSO- $\left.d_{6}, 200.13 \mathrm{MHz}, \mathrm{ppm}\right): \delta$ major isomer: $8.97\left(\mathrm{dd}, 2 \mathrm{H},{ }^{3} \mathrm{~J}_{\mathrm{H} 6, \mathrm{H} 5}=5.5 \mathrm{~Hz},{ }^{4} J_{\mathrm{H} 6, \mathrm{H} 4}=1.6 \mathrm{~Hz}\right.$, bpy-H6), $8.54\left(\mathrm{~d}, 2 \mathrm{H},{ }^{3} \mathrm{~J}_{\mathrm{H} 3, \mathrm{H} 4}=8.0 \mathrm{~Hz}\right.$, bpy-H3), $8.16\left(\mathrm{dt}, 2 \mathrm{H},{ }^{3} \mathrm{~J}_{\mathrm{H} 4, \mathrm{H} 3 / \mathrm{H} 5}=\right.$ $8.0 \mathrm{~Hz},{ }^{4} J_{\mathrm{H} 4, \mathrm{H} 6}=1.6 \mathrm{~Hz}$, bpy-H4), 7.58 (ddd, $2 \mathrm{H},{ }^{3} J_{\mathrm{H} 5, \mathrm{H} 4}=8.0 \mathrm{~Hz}$, $\left.{ }^{3} J_{\mathrm{H} 5, \mathrm{H} 6}=5.5 \mathrm{~Hz},{ }^{4} J_{\mathrm{H} 5, \mathrm{H} 3}=1.6 \mathrm{~Hz}, \mathrm{bpy}-\mathrm{H} 5\right), 3.63\left(\mathrm{~s}, 6 \mathrm{H}, \mathrm{COOCH}_{3}\right)$, 3.31-3.33 $\left(\mathrm{m}, 2 \mathrm{H}\right.$, allyl- $\mathrm{H}_{\text {syn, }}$ overlapping with water signal), 3.06-3.21 (m, $1 \mathrm{H}$, allyl- $\left.\mathrm{H}_{\text {meso }}\right), 1.49\left(\mathrm{~d}, 2 \mathrm{H},{ }^{3} J_{\text {Hanti,Hmeso }}=9.0 \mathrm{~Hz}\right.$, allyl- $\left.\mathrm{H}_{\text {anti }}\right)$; minor isomer: $9.19(\mathrm{~s}, 2 \mathrm{H}$, bpy-H6), 8.52-8.57 (m, $2 \mathrm{H}$, bpy-H3), 8.18-8.25 (m, 2H, bpy-H4), 7.77-7.84 (m, $2 \mathrm{H}$, bpy-H5), 4.05-4.14 (m, $1 \mathrm{H}$, allyl- $\left.\mathrm{H}_{\text {meso }}\right), 3.62\left(\mathrm{~s}, 6 \mathrm{H}, \mathrm{COOCH}_{3}\right)$, $3.45\left(\mathrm{~d}, 2 \mathrm{H},{ }^{3} J_{\text {Hsyn,Hanti }}=6.3 \mathrm{~Hz}\right.$, allyl- $\left.\mathrm{H}_{\text {syn }}\right), 1.47-1.54(\mathrm{~m}, 2 \mathrm{H}$, allyl- $\mathrm{H}_{\text {anti }}$ ); ${ }^{13} \mathrm{CNMR}$ (DMSO- $d_{6}, 50.32 \mathrm{MHz}, \mathrm{ppm}$ ): $\delta$ major isomer: 226.31 ( $C \equiv O), 162.38$ ( $\mathrm{C}=0), 154.49$ (bpy-C2), 152.24 (bpy-C6), 139.55 (bpy-C4), 138.34 (triazolate-C4/C5), 126.36 (bpy-C5), 122.76 (bpy-C3), 81.58 ( $\mathrm{CH}$-allyl), $57.56\left(\mathrm{CH}_{2}\right.$-allyl), $51.64\left(\mathrm{COOCH}_{3}\right)$; minor isomer: the signal intensity was below the detection limit.

[Mo( $\eta^{3}$-allyl)(triazolate $\left.{ }^{\mathrm{CF} 3, \mathrm{COOEt}}\right)(\mathrm{bpy})(\mathrm{CO})_{2}$ ]

$\left[\mathrm{Mo}\left(\eta^{3}\right.\right.$-allyl) $\left.\left(\mathrm{N}_{3}\right)(\mathrm{bpy})(\mathrm{CO})_{2}\right](2,44 \mathrm{mg}, 0.11 \mathrm{mmol})$ and $4,4,4-$ trifluoro-2-butynoic acid ethyl ester $(23 \mathrm{mg}, 0.14 \mathrm{mmol})$ were dissolved in dichloromethane $(15 \mathrm{~mL})$ and stirred at room temperature under exclusion of light for $4 \mathrm{~d}$. Then, the solvent was removed under vacuum and the red residue washed with $n$-hexane $(3 \times 5 \mathrm{~mL})$. After drying under vacuum, the product was obtained as a red solid. Yield: $55 \%(35 \mathrm{mg}, 0.06 \mathrm{mmol}$ ). Elemental analysis (\%): calc. $\mathrm{C}_{21} \mathrm{H}_{18} \mathrm{~F}_{3} \mathrm{MoN}_{5} \mathrm{O}_{4}$ : C 45.26, H 3.26, N 12.57, found: C 45.26, H 3.34, N 12.38; IR (ATR, cm $\left.{ }^{-1}\right)$ : 1938

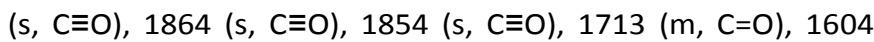
$(\mathrm{m}), 1472(\mathrm{w}), 1441(\mathrm{~m}), 1310(\mathrm{~m}), 1161(\mathrm{~m}), 1132(\mathrm{~m}), 1049$ (m), $763(\mathrm{w}), 734(\mathrm{~m}) ;{ }^{1} \mathrm{H}$ NMR (DMSO-d $\left.d_{6}, 199.93 \mathrm{MHz}, \mathrm{ppm}\right): \delta$ major isomer: $8.79\left(\mathrm{dd}, 2 \mathrm{H},{ }^{3} \mathrm{~J}_{\mathrm{H} 6, \mathrm{H} 5}=5.3 \mathrm{~Hz},{ }^{4} J_{\mathrm{H} 6, \mathrm{H} 4}=0.9 \mathrm{~Hz}\right.$, bpy-H6), $8.56\left(\mathrm{~d}, 2 \mathrm{H},{ }^{3} J_{\mathrm{H} 3, \mathrm{H} 4}=8.2 \mathrm{~Hz}, \mathrm{bpy}-\mathrm{H} 3\right), 8.18(\mathrm{dt}, 2 \mathrm{H}$, $\left.{ }^{3} J_{\mathrm{H} 4, \mathrm{H} 3 / \mathrm{H} 5}=7.9 \mathrm{~Hz},{ }^{4} J_{\mathrm{H} 4, \mathrm{H} 6}=1.5 \mathrm{~Hz}, \mathrm{bpy}-\mathrm{H} 4\right), 7.58$ (ddd, $2 \mathrm{H},{ }^{3} J_{\mathrm{H} 5, \mathrm{H} 4}$ $\left.=7.6 \mathrm{~Hz},{ }^{3} J_{\mathrm{H} 5, \mathrm{H} 6}=5.5 \mathrm{~Hz},{ }^{4} J_{\mathrm{H} 5, \mathrm{H} 3}=1.1 \mathrm{~Hz}, \mathrm{bpy}-\mathrm{H} 5\right), 4.14$ (q, $2 \mathrm{H},{ }^{3} \mathrm{~J}$ $\left.=7.2 \mathrm{~Hz}, \mathrm{COOCH}_{2} \mathrm{CH}_{3}\right), 1.52\left(\mathrm{~d}, 2 \mathrm{H},{ }^{3} J_{\text {Hanti,Hmeso }}=9.0 \mathrm{~Hz}\right.$, allyl$\left.\mathrm{H}_{\text {anti }}\right), 1.18\left(\mathrm{t}, 3 \mathrm{H},{ }^{3} \mathrm{~J}=7.1 \mathrm{~Hz}, \mathrm{COOCH}_{2} \mathrm{CH}_{3}\right)$, allyl- $\mathrm{H}_{\text {meso }}$ and allyl$\mathrm{H}_{\text {syn, }}$ overlapping with solvent water signal; minor isomer not observed due to low signal intensity; ${ }^{13} \mathrm{C}$ NMR (DMSO- $d_{6}, 50.27$ $\mathrm{MHz}, \mathrm{ppm}$ ): $\delta$ major isomer: 154.71 (bpy-C2), 152.18 (bpy-C6), 139.62 (bpy-C4), 126.33 (bpy-C5), 122.72 (bpy-C3), 60.23 $\left(\mathrm{COOCH}_{2} \mathrm{CH}_{3}\right), 57.61\left(\mathrm{CH}_{2}\right.$-allyl), $13.88\left(\mathrm{COOCH}_{2} \mathrm{CH}_{3}\right), \mathrm{CO}$ and $\mathrm{CH}$-allyl signals not observed due to low signal intensity; minor isomer: the signal intensity was below the detection limit; ${ }^{19} \mathrm{~F}$ NMR (DMSO- $\left.d_{6}, 188.12 \mathrm{MHz}, \mathrm{ppm}\right): \delta-58.76\left(\mathrm{CF}_{3}\right.$, major species, $80 \%),-58.56\left(\mathrm{CF}_{3}\right.$, minor species, $\left.14 \%\right),-57.53\left(\mathrm{CF}_{3}\right.$, minor species, 6\%).

$\left[\mathbf{W}\left(\eta^{3} \text {-allyl)Cl(bpy)(CO }\right)_{2}\right]$ (9). To a degassed mixture of anhydrous tetrahydrofuran $(35 \mathrm{~mL})$ and allyl chloride $(4.98 \mathrm{~mL}$, $4.68 \mathrm{~g}, 61.2 \mathrm{mmol})$, solid $\left[\mathrm{W}(\mathrm{bpy})(\mathrm{CO})_{3}(\mathrm{py})\right](8,1.03 \mathrm{~g}, 2.05$ $\mathrm{mmol}$ ) was added and the reaction mixture heated to reflux for $4.5 \mathrm{~h}$. The resulting dark brown precipitate was filtered from the dark violet solution, washed with $n$-hexane $(50 \mathrm{~mL})$, and dried under vacuum. Yield: $86 \%$ ( $836 \mathrm{mg}, 1.77 \mathrm{mmol}$ ). Elemental analysis (\%): calc. $\mathrm{C}_{15} \mathrm{H}_{13} \mathrm{ClN}_{2} \mathrm{O}_{2} \mathrm{~W} \cdot \mathrm{H}_{2} \mathrm{O}: \mathrm{C} 38.12, \mathrm{H}$ 
2.77, N 5.93, found: C 36.02, H 3.09, N 5.84; IR (ATR, $\mathrm{cm}^{-1}$ ) $2976(\mathrm{~m}), 1915(\mathrm{~s}), 1819(\mathrm{~s}), 1601(\mathrm{~m}), 1466(\mathrm{~m}), 1445(\mathrm{~m}), 773$ (s); ${ }^{1} \mathrm{H}$ NMR (DMSO- $d_{6}, 500.13 \mathrm{MHz}, \mathrm{ppm}$ ): $\delta$ major isomer: 8.81 (ddd, $2 \mathrm{H},{ }^{3} J_{\mathrm{H} 6, \mathrm{H} 5}=5.5 \mathrm{~Hz},{ }^{4} J_{\mathrm{H} 6, \mathrm{H} 4}=1.5 \mathrm{~Hz},{ }^{5} J_{\mathrm{H} 6, \mathrm{H} 3}=0.6 \mathrm{~Hz}$, bpy-H6), $8.64\left(\mathrm{~d}, 2 \mathrm{H},{ }^{3} J_{\mathrm{H} 3, \mathrm{H} 4}=8.2 \mathrm{~Hz}, \mathrm{bpy}-\mathrm{H} 3\right), 8.24(\mathrm{dt}, 2 \mathrm{H}$, $\left.{ }^{3} J_{\mathrm{H} 4, \mathrm{H} 3 / \mathrm{H} 5}=7.9 \mathrm{~Hz},{ }^{4} J_{\mathrm{H} 4, \mathrm{H} 6}=1.6 \mathrm{~Hz}, \mathrm{bpy}-\mathrm{H} 4\right), 7.67$ (ddd, $2 \mathrm{H},{ }^{3} J_{\mathrm{H} 5, \mathrm{H} 4}$ $=7.6 \mathrm{~Hz},{ }^{3} J_{\mathrm{H} 5, \mathrm{H} 6}=5.5 \mathrm{~Hz},{ }^{4} J_{\mathrm{H} 5, \mathrm{H} 3}=1.2 \mathrm{~Hz}$, bpy-H5), $2.85(\mathrm{~d}, 2 \mathrm{H}$, ${ }^{3} J_{\text {Hsyn,Hmeso }}=6.0 \mathrm{~Hz}$, allyl- $\left.\mathrm{H}_{\text {syn }}\right), 2.29-2.37\left(\mathrm{~m}, 1 \mathrm{H}\right.$, allyl- $\left.\mathrm{H}_{\text {meso }}\right)$, $1.45\left(\mathrm{~d}, 2 \mathrm{H},{ }^{3} J_{\text {Hanti,Hmeso }}=8.3 \mathrm{~Hz}\right.$, allyl- $\left.\mathrm{H}_{\text {anti }}\right)$; minor isomer: 9.07 $(\mathrm{m}, 2 \mathrm{H}, \mathrm{bpy}-\mathrm{H} 6), 8.72\left(\mathrm{~d}, 2 \mathrm{H},{ }^{3} \mathrm{~J}_{\mathrm{H} 3, \mathrm{H} 4}=8.1 \mathrm{~Hz}, \mathrm{bpy}-\mathrm{H} 3\right), 8.28(\mathrm{dt}$, $2 \mathrm{H},{ }^{3} J_{\mathrm{H} 4, \mathrm{H} 3 / \mathrm{H} 5}=7.8 \mathrm{~Hz},{ }^{4} J_{\mathrm{H} 4, \mathrm{H} 6}=1.3 \mathrm{~Hz}$, bpy-H4), $7.82(\mathrm{t}, 2 \mathrm{H}$, ${ }^{3} J_{\mathrm{H} 5, \mathrm{H} 4 / \mathrm{H} 6}=6.4 \mathrm{~Hz}$, bpy-H5), $3.30\left(\mathrm{~m}, 2 \mathrm{H}\right.$, allyl- $\left.\mathrm{H}_{\text {syn }}\right), 3.05-3.14$ $\left(\mathrm{m}, 1 \mathrm{H}\right.$, allyl- $\left.\mathrm{H}_{\text {meso }}\right), 1.50\left(\mathrm{~d}, 2 \mathrm{H},{ }^{3} \mathrm{~J}_{\text {Hanti,Hmeso }}=8.0 \mathrm{~Hz}\right.$, allyl- $\left.\mathrm{H}_{\text {anti }}\right)$; ${ }^{13} \mathrm{C}$ NMR (DMSO- $d_{6}, 125.76 \mathrm{MHz}, \mathrm{ppm}$ ): $\delta$ major isomer: 220.52 (C三O), 154.17 (bpy-C2), 152.02 (bpy-C6), 139.36 (bpy-C4), 127.03 (bpy-C5), 123.44 (bpy-C3), 62.96 ( $\mathrm{CH}$-allyl), $45.91\left(\mathrm{CH}_{2-}\right.$ allyl); minor isomer: the signal intensity was below the detection limit.

[W( $\eta^{3}$-allyl)( $\left.\mathbf{N}_{3}\right)($ bpy $\left.)(\mathbf{C O})_{2}\right]$ (10). In a degassed mixture of methanol $(100 \mathrm{~mL})$ and acetone $(25 \mathrm{~mL})$, solid $\left[\mathrm{W}\left(\eta^{3}\right.\right.$ allyl)Cl(bpy) $\left.(\mathrm{CO})_{2}\right](9,300 \mathrm{mg}, 0.63 \mathrm{mmol})$ and sodium azide (207 $\mathrm{mg}, 3.18 \mathrm{mmol}$ ) were dissolved under argon and the reaction mixture heated to reflux for $6 \mathrm{~h}$. The resulting precipitate was filtered off from the dark red solution, washed with water $(80 \mathrm{~mL})$ and methanol $(40 \mathrm{~mL})$, and dried under vacuum to result in a red-brown solid. Yield: $62 \%$ (189 mg, 0.39 mmol). Elemental analysis (\%): calc. $\mathrm{C}_{15} \mathrm{H}_{13} \mathrm{~N}_{5} \mathrm{O}_{2} \mathrm{~W}: \mathrm{C} 37.60, \mathrm{H}$ 2.73, N 14.62, found: C 37.57, H 2.70, N 14.57; IR (ATR, $\mathrm{cm}^{-1}$ ): 2046 (vs), 1920 (s), 1821 (vs), 1602 (m), 1471 (m), 1442 (m), $756(\mathrm{~m}) ;{ }^{1} \mathrm{H}$ NMR (DMSO- $d_{6}, 500.13 \mathrm{MHz}, \mathrm{ppm}$ ): $\delta$ major isomer: 8.84 (ddd, $2 \mathrm{H},{ }^{3} \mathrm{~J}_{\mathrm{H} 6, \mathrm{H} 5}=5.4 \mathrm{~Hz},{ }^{4} \mathrm{~J}_{\mathrm{H} 6, \mathrm{H} 4}=1.4 \mathrm{~Hz},{ }^{5} J_{\mathrm{H} 6, \mathrm{H} 3}=$ $0.5 \mathrm{~Hz}, \mathrm{bpy}-\mathrm{H} 6), 8.64\left(\mathrm{~d}, 2 \mathrm{H},{ }^{3} J_{\mathrm{H} 3, \mathrm{H} 4}=8.2 \mathrm{~Hz}, \mathrm{bpy}-\mathrm{H} 3\right), 8.27$ (dt, $2 \mathrm{H},{ }^{3} J_{\mathrm{H} 4, \mathrm{H} 3 / \mathrm{H} 5}=7.9 \mathrm{~Hz},{ }^{4} J_{\mathrm{H} 4, \mathrm{H} 6}=1.6 \mathrm{~Hz}$, bpy-H4), 7.70 (ddd, $2 \mathrm{H}$, $\left.{ }^{3} J_{\mathrm{H} 5, \mathrm{H} 4}=7.6 \mathrm{~Hz},{ }^{3} J_{\mathrm{H} 5, \mathrm{H} 6}=5.5 \mathrm{~Hz},{ }^{4} J_{\mathrm{H} 5, \mathrm{H} 3}=1.2 \mathrm{~Hz}, \mathrm{bpy}-\mathrm{H} 5\right), 2.95$ (d, $2 \mathrm{H},{ }^{3} J_{\text {Hsyn,Hmeso }}=6.2 \mathrm{~Hz}$, allyl- $\left.\mathrm{H}_{\text {syn }}\right), 2.35-2.43(\mathrm{~m}, 1 \mathrm{H}$, allyl$\left.\mathrm{H}_{\text {meso }}\right), 1.48\left(\mathrm{~d}, 2 \mathrm{H},{ }^{3}\right.$ Hanti,Hmeso $=8.7 \mathrm{~Hz}$, allyl- $\left.\mathrm{H}_{\text {anti }}\right)$; minor isomer: $9.07(\mathrm{~m}, 2 \mathrm{H}$, bpy- $\mathrm{H} 6), 8.75\left(\mathrm{~d}, 2 \mathrm{H},{ }^{3} \mathrm{~J}_{\mathrm{H} 3, \mathrm{H} 4}=8.0 \mathrm{~Hz}\right.$, bpyH3), $8.32\left(\mathrm{dt}, 2 \mathrm{H},{ }^{3} J_{\mathrm{H} 4, \mathrm{H} 3 / \mathrm{H} 5}=7.7 \mathrm{~Hz},{ }^{4} J_{\mathrm{H} 4, \mathrm{H} 6}=1.2 \mathrm{~Hz}\right.$, bpy$\mathrm{H} 4), 7.86\left(\mathrm{t}, 2 \mathrm{H},{ }^{3} \mathrm{~J}_{\mathrm{H} 5, \mathrm{H} 4 / \mathrm{H} 6}=6.2 \mathrm{~Hz}, \mathrm{bpy}-\mathrm{H} 5\right), 3.18(\mathrm{~d}, 2 \mathrm{H}$, ${ }^{3} J_{\text {Hsyn,Hmeso }}=6.1 \mathrm{~Hz}$, allyl- $\left.\mathrm{H}_{\text {syn }}\right), 3.02-3.13\left(\mathrm{~m}, 1 \mathrm{H}\right.$, allyl- $\left.\mathrm{H}_{\text {meso }}\right)$, $1.51\left(\mathrm{~d}, 2 \mathrm{H},{ }^{3} \mathrm{~J}_{\text {Hanti,Hmeso }}=9.0 \mathrm{~Hz}\right.$, allyl- $\left.\mathrm{H}_{\text {anti }}\right) ;{ }^{13} \mathrm{C}$ NMR (DMSO- $d_{6}$, $125.76 \mathrm{MHz}, \mathrm{ppm}$ ): $\delta$ major isomer: 220.57 (C三O), 154.14 (bpyC2), 152.06 (bpy-C6), 139.70 (bpy-C4), 127.30 (bpy-C5), 123.44 (bpy-C3), $65.12\left(\mathrm{CH}\right.$-allyl), $47.91\left(\mathrm{CH}_{2}\right.$-allyl); minor isomer: the signal intensity was below the detection limit.

[W( $\eta^{3}$-allyl)(triazolate $\left.\left.{ }^{\mathrm{COOCH} 3, \mathrm{COOCH}_{3}}\right)(\mathrm{bpy})(\mathrm{CO})_{2}\right]$

(11).

$\left[\mathrm{W}\left(\eta^{3}\right.\right.$-allyl) $\left(\mathrm{N}_{3}\right)($ bpy $\left.)(\mathrm{CO})_{2}\right] \quad(\mathbf{1 0}, 30 \mathrm{mg}, 0.06 \mathrm{mmol})$ was dissolved in dichloromethane $(3 \mathrm{~mL})$. Dimethyl acetylenedicarboxylate (DMAD, $31 \mu \mathrm{L}, 35.6 \mathrm{mg}, 0.25 \mathrm{mmol}$ ) was added and the reaction mixture stirred at room temperature for $5 \mathrm{~d}$. Then, diethyl ether $(50 \mathrm{~mL})$ was added to precipitate the product from the red-brown solution. The resulting brown crystalline solid was filtered off, washed with diethyl ether (10 $\mathrm{mL}$ ), and dried under vacuum. Yield: $83 \%$ (33 $\mathrm{mg}, 0.05 \mathrm{mmol}$ ). Elemental analysis (\%): calc. $\mathrm{C}_{21} \mathrm{H}_{19} \mathrm{~N}_{5} \mathrm{O}_{6} \mathrm{~W}: \mathrm{C} 40.60, \mathrm{H} 3.08, \mathrm{~N}$ 11.27, found: 39.87, H 3.29, N 10.82; IR (ATR, $\mathrm{cm}^{-1}$ ): 1921 (s), 1841 (s), 1724 (s); ${ }^{1} \mathrm{H}$ NMR (DMSO- $\left.d_{6}, 500.13 \mathrm{MHz}, \mathrm{ppm}\right): \delta$ major isomer: $8.82\left(\mathrm{dd}, 2 \mathrm{H},{ }^{3} \mathrm{~J}_{\mathrm{H} 6, \mathrm{H} 5}=5.5 \mathrm{~Hz},{ }^{4} J_{\mathrm{H} 6, \mathrm{H} 4}=0.9 \mathrm{~Hz}\right.$, bpy-H6), $8.61\left(\mathrm{~d}, 2 \mathrm{H},{ }^{3} \mathrm{~J}_{\mathrm{H} 3, \mathrm{H} 4}=8.2 \mathrm{~Hz}, \mathrm{bpy}-\mathrm{H} 3\right), 8.24(\mathrm{dt}, 2 \mathrm{H}$, ${ }^{3} J_{\mathrm{H} 4, \mathrm{H} 3 / \mathrm{H} 5}=7.9 \mathrm{~Hz},{ }^{4} J_{\mathrm{H} 4, \mathrm{H} 6}=1.5 \mathrm{~Hz}$, bpy-H4), 7.61 (ddd, $2 \mathrm{H},{ }^{3} J_{\mathrm{H} 5, \mathrm{H} 4}$ $\left.=7.5 \mathrm{~Hz},{ }^{3} J_{\mathrm{H} 5, \mathrm{H} 6}=5.5 \mathrm{~Hz},{ }^{4} J_{\mathrm{H} 5, \mathrm{H3}}=1.1 \mathrm{~Hz}, \mathrm{bpy}-\mathrm{H} 5\right), 3.63(\mathrm{~s}, 6 \mathrm{H}$, $\left.\mathrm{COOCH}_{3}\right), 3.10\left(\mathrm{~d}, 2 \mathrm{H},{ }^{3} \mathrm{~J}_{\mathrm{Hsyn}, \mathrm{Hmeso}}=6.2 \mathrm{~Hz}\right.$, allyl- $\left.\mathrm{H}_{\text {syn }}\right), 2.29-2.37$ $\left(\mathrm{m}, 1 \mathrm{H}\right.$, allyl- $\left.\mathrm{H}_{\text {meso }}\right), 1.68\left(\mathrm{~d}, 2 \mathrm{H},{ }^{3} J_{\text {Hanti,Hmeso }}=8.8 \mathrm{~Hz}\right.$, allyl- $\left.\mathrm{H}_{\text {anti }}\right)$; minor isomer: $3.59\left(\mathrm{~s}, \mathrm{COOCH}_{3}\right)$, the other signals were too weak for clear identification; ${ }^{13} \mathrm{C}$ NMR (DMSO- $d_{6}, 125.76 \mathrm{MHz}$, ppm): $\delta 220.04$ (C=O), $162.12\left(\mathrm{COOCH}_{3}\right), 155.53$ (bpy-C2), 152.50 (bpy-C6), 139.83 (bpy-C4), 138.83 (triazolate-C4/C5), 127.19 (bpy-C5), 123.15 (bpy-C3), 65.48 (CH-allyl), 51.79 $\left(\mathrm{COOCH}_{3}\right), 49.45\left(\mathrm{CH}_{2}\right.$-allyl); minor isomer: the signal intensity was below the detection limit.

[W( $\eta^{3}$-allyl)(triazolate $\left.\left.{ }^{\mathrm{CF} 3, \mathrm{COOEt}}\right)(\mathrm{bpy})(\mathrm{CO})_{2}\right]$

$\left[\mathrm{W}\left(\eta^{3}\right.\right.$-allyl $\left.)\left(\mathrm{N}_{3}\right)(\mathrm{bpy})(\mathrm{CO})_{2}\right] \quad(\mathbf{1 0}, 25 \mathrm{mg}, 0.05 \mathrm{mmol})$ was dissolved in dichloromethane $(5 \mathrm{~mL})$. 4,4,4-Trifluoro-2butynoic acid ethyl ester $(17 \mathrm{mg}, 0.10 \mathrm{mmol}$ ) was added and the reaction mixture stirred at room temperature for $6 \mathrm{~d}$. Then, diethyl ether $(30 \mathrm{~mL})$ was added to precipitate the product from the dark red solution. The resulting red-brown crystalline solid was filtered off, washed with diethyl ether (10 $\mathrm{mL}$ ), and dried under vacuum. Yield: $40 \%$ (12 mg, $0.02 \mathrm{mmol}$ ). Elemental analysis (\%): calc. $\mathrm{C}_{21} \mathrm{H}_{18} \mathrm{~F}_{3} \mathrm{~N}_{5} \mathrm{O}_{4} \mathrm{~W}$ : C 39.09, $\mathrm{H} 2.81, \mathrm{~N}$ 10.85, found: 39.09, H 2.86, N 10.56; IR (ATR, $\mathrm{cm}^{-1}$ ): 1929 (s), 1850 (s), 1723 (s); ${ }^{1} \mathrm{H}$ NMR (DMSO- $d_{6}, 500.13 \mathrm{MHz}, \mathrm{ppm}$ ): $\delta$ major isomer: $8.83\left(\mathrm{dd}, 2 \mathrm{H},{ }^{3} J_{\mathrm{H} 6, \mathrm{H} 5}=5.4 \mathrm{~Hz},{ }^{4} J_{\mathrm{H} 6, \mathrm{H} 4}=0.8 \mathrm{~Hz}\right.$, bpy-H6), $8.64\left(\mathrm{~d}, 2 \mathrm{H},{ }^{3} \mathrm{~J}_{\mathrm{H} 3, \mathrm{H} 4}=8.2 \mathrm{~Hz}, \mathrm{bpy}-\mathrm{H} 3\right), 8.25(\mathrm{dt}, 2 \mathrm{H}$, ${ }^{3} J_{\mathrm{H} 4, \mathrm{H} 3 / \mathrm{H} 5}=7.9 \mathrm{~Hz},{ }^{4} J_{\mathrm{H} 4, \mathrm{H} 6}=1.6 \mathrm{~Hz}$, bpy-H4), 7.61 (ddd, $2 \mathrm{H},{ }^{3} J_{\mathrm{H} 5, \mathrm{H} 4}$ $=7.5 \mathrm{~Hz},{ }^{3} J_{\mathrm{H} 5, \mathrm{H} 6}=5.5 \mathrm{~Hz},{ }^{4} J_{\mathrm{H} 5, \mathrm{H} 3}=1.1 \mathrm{~Hz}$, bpy-H5), $4.14\left(\mathrm{q}, 2 \mathrm{H},{ }^{3}\right.$ J $\left.=7.1 \mathrm{~Hz}, \mathrm{COOCH}_{2} \mathrm{CH}_{3}\right), 3.13\left(\mathrm{~d}, 2 \mathrm{H},{ }^{3} J_{\text {Hsyn, Hmeso }}=6.2 \mathrm{~Hz}\right.$, allyl$\left.\mathrm{H}_{\text {syn }}\right), 2.37-2.44\left(\mathrm{~m}, 1 \mathrm{H}\right.$, allyl- $\left.\mathrm{H}_{\text {meso }}\right), 1.71\left(\mathrm{~d}, 2 \mathrm{H},{ }^{3} J_{\text {Hanti,Hmeso }}=\right.$ $8.8 \mathrm{~Hz}$, allyl- $\left.\mathrm{H}_{\text {anti }}\right), 1.17\left(\mathrm{t}, 3 \mathrm{H},{ }^{3} \mathrm{~J}=7.1 \mathrm{~Hz}, \mathrm{COOCH}_{2} \mathrm{CH}_{3}\right)$; minor isomer: signals were too weak for clear identification; ${ }^{13} \mathrm{C} \mathrm{NMR}$ (DMSO- $d_{6}, 125.76 \mathrm{MHz}, \mathrm{ppm}$ ): $\delta$ major isomer: 219.88 (C三O), $159.69\left(\mathrm{COOCH}_{2} \mathrm{CH}_{3}\right), 155.70$ (bpy-C2), 152.45 (bpy-C6), 139.85 (bpy-C4), $137.07\left(\mathrm{q},{ }^{2} \mathrm{~J}_{\mathrm{C}, \mathrm{F}}=37.1 \mathrm{~Hz}\right.$, triazolate-C4), $136.18(\mathrm{~m}$, ${ }^{3} J_{C, F}<0.6 \mathrm{~Hz}$, triazolate-C5), 127.13 (bpy-C5), 123.09 (bpy-C3), $121.09\left(\mathrm{q},{ }^{1} J_{\mathrm{C}, \mathrm{F}}=268 \mathrm{~Hz}, \mathrm{CF}_{3}\right), 65.39(\mathrm{CH}$-allyl $), 60.39$ $\left(\mathrm{COOCH}_{2} \mathrm{CH}_{3}\right), 49.50\left(\mathrm{CH}_{2}\right.$-allyl), $13.85\left(\mathrm{COOCH}_{2} \mathrm{CH}_{3}\right)$; minor isomer: the signal intensity was below the detection limit; ${ }^{19} \mathrm{~F}$ NMR (DMSO- $\left.d_{6}, 470.59 \mathrm{MHz}, \mathrm{ppm}\right): \delta-58.95\left(\mathrm{CF}_{3}\right.$, major species, $88 \%),-58.70\left(\mathrm{CF}_{3}\right.$, minor species, $\left.12 \%\right)$.

\section{$\mathrm{X}$-ray diffraction analysis}

Single crystals suitable for X-ray structure determination of $\mathbf{2}$, $5,6,11$, and 12 were obtained by slow diffusion of $n$-hexane into a solution of the compound in dichloromethane while $\mathbf{1 0}$ was recrystallized from a mixture of acetone and methanol. Selected crystals were immersed in a film of perfluoropolyether oil, mounted on a polyimide microloop (MicroMounts, MiTeGen) or glass fiber, and transferred to a stream of cold dinitrogen (Bruker Kryoflex2). Crystal data for 2, 6, 10, 11 and 12 were obtained on a BRUKER X8-APEX II while data for $\mathbf{5}$ was collected on a BRUKER SMART-APEX diffractometer. Both instruments were equipped with a CCD area detector and used graphite monochromated $\mathrm{Mo}_{\mathrm{K} \alpha}$ radiation. The structures were solved using the intrinsic 
phasing method, ${ }^{64}$ refined with the SHELXL program ${ }^{65}$ and expanded using Fourier techniques. All non-hydrogen atoms were refined anisotropically. Hydrogen atoms were included in structure factors calculations. All hydrogen atoms, except those of the allyl moiety in some cases, were assigned to idealised positions. The coordinates of the hydrogen atoms of the allyl moiety were refined freely. The $\mathrm{C}-\mathrm{H}$ distances in the allyl moiety were restrained to a values of 0.950 (5) $\AA$ during the refinement. Crystallographic data has been deposited with the Cambridge Crystallographic Data Centre as supplementary publications no. CCDC-1562700-1562705. These data can be obtained free of charge from the Cambridge Crystallographic Data Centre via www.ccdc.cam.ac.uk/data_request/cif.

\section{DFT calculations}

DFT calculations were carried out on the Linux cluster of the Leibniz-Rechenzentrum (LRZ) in Munich with ORCA version $2.8{ }^{66}$ using the BP86 functional with the resolution-of-theidentity (RI) approximation, a def2-TZVP/def2-TZVP/J basis set, the tightscf and grid4 options, and the COSMO solvation model with dimethylsulfoxide as the solvent for geometry optimization and subsequent calculation of vibrational frequencies to characterize the structure obtained as a minimum by inspection for absence of imaginary modes. Then, a relaxed surface scan was carried out in $15^{\circ}$ steps for the full $360^{\circ}$ rotation of the triazolate ring relative to the central $\mathrm{C}-\mathrm{C} 2$ axis of the 2,2'-bipyridine ligand while allowing all other variables to relax. The maxima located on the resulting potential energy curve where then further characterized as transition states using the optTS keyword in separate runs.

\section{Conflicts of interest}

There are no conflicts of interest to declare.

\section{Acknowledgements}

This work was supported by the Deutsche Forschungsgemeinschaft (DFG) with grants no. SCHA962/3-1 and SCHA962/8-1 to U.S.

\section{References}

1 H. C. Kolb, M. G. Finn and K. B. Sharpless, Angew. Chem. Int. Ed., 2001, 40, 2004.

2 H. C. Kolb and K. B. Sharpless, Drug Discovery Today, 2003, 8, 1128.

3 J. E. Moses and A. D. Moorhouse, Chem. Soc. Rev., 2007, 36, 1249.

4 P. Thirumurugan, D. Matosiuk and K. Jozwiak, Chem. Rev., 2013, 113, 4905.

5 J. A. Prescher and C. R. Bertozzi, Nature Chem. Biol., 2005 1, 13.

6 C. R. Bertozzi and E. M. Sletten, Angew. Chem. Int. Ed., 2009, 48, 6974.

7 R. K. V. Lim and Q. Lin, Chem. Commun., 2010, 46, 1589.
M. Grammel and H. C. Hang, Nature Chem. Biol., 2013, 9, 475.

C. P. Ramil and Q. Lin, Chem. Commun., 2013, 49, 11007.

K. Lang and J. W. Chin, Chem. Rev., 2014, 114, 4764.

N. K. Devaraj and R. Weissleder, Acc. Chem. Res., 2011, 44, 816.

T. J. Del Castillo, S. Sarkar, K. A. Abboud and A. S. Veige, Dalton Trans., 2011, 40, 8140.

L. Cararrubios, M. C. de la Torre and M. A. Sierra, Chem. Eur. J., 2013, 19, 3534.

H.-W. Frühauf, Chem. Rev., 1997, 97, 523.

H.-W. Frühauf, Coord. Chem. Rev., 2002, 230, 79.

W. P. Fehlhammer and W. Beck, Z. Anorg. Allg. Chem., 2015, 641, 1599.

C. C. Beto, E. D. Holt, Y. Yang, I. Ghiviriga, K. S. Schanze and A. S. Veige, Chem. Commun., 2017, 53, 9934.

A. R. Powers, I. Ghiviriga, K. A. Abboud and A. S. Veige, Dalton Trans., 2015, 44, 14747.

L. Waag-Hiersch, J. Mößeler and U. Schatzschneider, Eur. J. Inorg. Chem., 2017, 3024.

J. A. Krause-Bauer, T. M. Becker and M. Orchin, J. Chem. Cryst., 2004, 34, 843.

K. Pachhunga, B. Therrien and M. R. Kollipara, Inorg. Chim. Acta, 2008, 361, 3294.

F.-C. Liu, Y.-L. Lin, P.-S. Yang, G.-H. Lee and S.-M. Peng, Organometallics, 2010, 29, 4282.

K. Agapiou, R. A. Jones and X. Yang, Main Group Chem., 2010, 9, 41.

Y.-H. Lo, T.-H. Wang, C.-Y. Lee and Y.-H. Feng, Organometallics, 2012, 31, 6887.

S. Miguel-Fernandez, S. M. de Salinas, J. Diez, M. P. Gamasa and E. Lastra, Inorg. Chem., 2013, 52, 4293.

F.-C. Liu, J.-E. Liang, J.-Y. Jin, Y.-L. Lin, Y.-J. Chu, P.-S. Yang, G.-H. Lee and S.-M. Peng, J. Organomet. Chem., 2013, 735, 1.

T. Cruchter, K. Harms and E. Meggers, Chem. Eur. J., 2013, 19, 16682.

L. Henry, C. Schneider, B. Mützel, P. V. Simpson, C. Nagel, K. Fucke and U. Schatzschneider, Chem. Commun., 2014, 50, 15692.

K. S. Singh, K. A. Kreisel, G. P. A. Yap and M. R. Kollipara, J. Organomet. Chem., 2006, 691, 3509.

H. Pfeiffer, M. Dragoun, A. Prokop and U. Schatzschneider, Z. Anorg. Allg. Chem., 2013, 639, 1568.

T. S. A. Hor and S.-M. Chee, J. Organomet. Chem., 1987, 331, 23.

H. Behrens and N. Harder, Chem. Ber., 1964, 97, 433.

S. S. van Berkel, T. J. Dirks, M. F. Debets, F. L. van Delft, J. J. L. M. Cornelissen, R. J. M. Nolte and F. P. J. T. Rutjes, ChemBioChem, 2007, 8, 1504.

J. E. Bunch and C. L. Bumgardner, J. Fluorine Chem., 1987, 36, 313.

B. C. Hamper, Org. Synth., 1992, 70, 246.

Y. Kobayashi, T. Yamashita, K. Takahashi, H. Kuroda and I. Kumadaki, Tetrahedron Lett., 1982, 23, 343.

G. Meazza and G. Zanardi, J. Fluorine Chem., 1991, 55, 199.

S. T. D. Gough and S. Trippett, J. Chem. Soc., 1962, 2333.

Y. Kobayashi, T. Yamashita, K. Takahashi, H. Kuroda and I. Kumadaki, Chem. Pharm. Bull., 1984, 32, 4402.

F. Eymery, B. lorga and P. Savignac, Synthesis, 2000, 185.

Z. Dori and R. F. Ziolo, Chem. Rev., 1973, 73, 247. 
J. W. Faller, D. A. Haitko, R. D. Adams and D. F. Chodosh, J. Am. Chem. Soc., 1979, 101, 865.

43 J. W. Faller and B. C. Whitmore, Organometallics, 1986, 5, 752.

44 M. J. Calhorda and P. J. Costa, Coord. Chem. Rev., 2017, 344, 83.

D. E. Ryan, D. J. Cardin and F. Hartl, Coord. Chem. Rev., 2017, 335, 103.

46 A. G. Orpen, L. Brammer, F. H. Allen, O. Kennard, D. G. Watson and R. Taylor, Dalton Trans., 1989, S1.

E. O. Fischer, D. Wittmann, D. Himmelreich, R. Cai, K. Ackermann and D. Neugebauer, Chem. Ber., 1982, 115, 3152.

D. Sellmann, W. Weber, G. Liehr and H. P. Beck, J. Organomet. Chem., 1984, 269, 155.

H. Wadle, E. Conradi, U. Müller and K. Dehnicke, Z. Naturforsch. B, 1985, 40, 1626.

D. Fenske, A. Frankenau and K. Dehnicke, Z. Anorg. Allg. Chem., 1989, 579, 27.

H.-W. Lam, G. Wilkinson, B. Hussian-Bates and M. B. Hursthouse, Dalton Trans., 1993, 781.

D. Rische, A. Baunemann, M. Winter and R. A. Fischer, Inorg. Chem., 2006, 45, 269.

F.-C. Liu, T.-C. Tsai, Y.-L. Lin, C.-S. Lee, P.-S. Yang and J.-C. Wang, J. Organomet. Chem., 2010, 695, 423.

R. Haiges, J. Skotnitzki, Z. Fang, D. A. Dixon and K. O. Christe, Angew. Chem. Int. Ed., 2015, 54, 9581.

R. Haiges, J. Skotnitzki, Z. Fang, D. A. Dixon and K. O. Christe, Angew. Chem. Int. Ed., 2015, 54, 15550.

J. Pérez, V. Riera, A. Rodríguez, R. López, T. L. Sordo, S. García-Granda, E. García-Rodríguez and A. Galindo, Organometallics, 2003, 22, 1540.

C. Cheng and J. L. Chou, Inorg. Chem., 1995, 34, 854. 165.

A. R. Powers, X. Yang, T. J. Del Castillo, I. Ghiviriga, K. A. Abboud and A. S. Veige, Dalton Trans., 2013, 42, 14963. E. Evangelino, N. P. Rath and L. M. Mirica, Dalton Trans., 2012, 41, 8010.

61 E. A. Giner, M. Gomez-Gallego, L. Casarrubios, M. C. de la Torre, C. R. de Arellano and M. A. Sierra, Inorg. Chem., 2017, 56, 2801.

62 E. Hirota, S. Saito and Y. Endo, J. Chem. Phys., 1979, 71, 1183.

63 G. R. Fulmer, A. J. M. Miller, N. H. Sherden, H. E. Gottlieb, A. Nudelman, B. M. Stoltz, J. E. Bercaw and K. I. Goldberg, Organometallics, 2010, 29, 2176.

64 G. M. Sheldrick, Acta Cryst. A, 2015, A71, 3.

65 G. M. Sheldrick, Acta Cryst. A, 2008, A64, 112.

66 F. Neese, WIREs Comput. Mol. Sci., 2011, 2, 73. 


\title{
Catalyst-free room-temperature iClick reaction of molybdenum(II) and tungsten(II) azide complexes with electron-poor alkynes: Structural preferences and kinetic studies
}

\author{
Paul Schmid, ${ }^{a}$ Matthias Maier, ${ }^{a}$ Hendrik Pfeiffer, ${ }^{a}$ Anja Belz,${ }^{a}$ Lucas Henry, ${ }^{a}$ \\ Alexandra Friedrich, ${ }^{a}$ Fabian Schönfeld, ${ }^{a}$ Katharina Edkins ${ }^{\mathrm{a}, \mathrm{b}}$ and Ulrich Schatzschneider ${ }^{* a}$ \\ ${ }^{\mathrm{a}}$ Institut für Anorganische Chemie, \\ Julius-Maximilians-Universität Würzburg, Am Hubland, D-97074 Würzburg, Germany \\ Fax: +499313184605 \\ eMail: ulrich.schatzschneider@uni-wuerzburg.de
${ }^{\mathrm{b}}$ School of Pharmacy, Queen's University Belfast, 97 Lisburn Road, BT9 7BL, United Kingdom

Supporting Information 


\section{Synthesis of ligands and precursors}

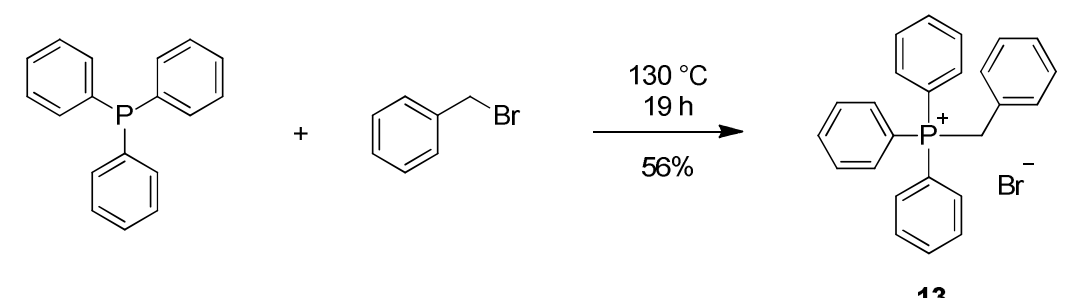

1) THF, $n B u L i,-10^{\circ} \mathrm{C}$

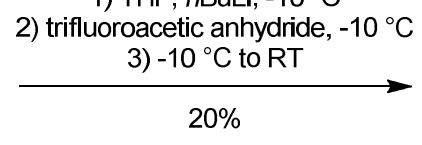

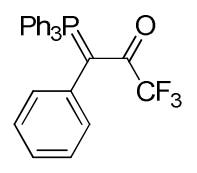

14

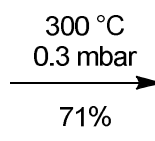

$71 \%$

Scheme S1. Three-step synthesis of phenyltrifluoromethylacetylene 15 from benzylbromide and triphenylphosphine (Y. Kobayashi, T. Yamashita, K. Takahashi, H. Kuroda and I. Kumadaki, Chem. Pharm. Bull. 1984, 32, 4402).

Synthesis of benzyltriphenylphosphonium bromide (13). Solid triphenylphosphine (15.3 g, $58.5 \mathrm{mmol})$ was mixed under argon with benzyl bromide $(7.6 \mathrm{~mL}, 11.0 \mathrm{~g}, 64.3$ mmol) and heated to $130{ }^{\circ} \mathrm{C}$ for $19 \mathrm{~h}$. The yellow crude product was then purified by column chromatography on silica using dichloromethane/methanol $(9: 1 \mathrm{v} / \mathrm{v})$ as the eluent to obtain a colorless solid. Yield: $56 \%(14.2 \mathrm{~g}, 32.8 \mathrm{mmol})$. Elemental analysis (\%): calc. $\mathrm{C}_{25} \mathrm{H}_{22}$ BrP: C 69.30, H 5.12, found: C 69.19, H 5.10; IR (ATR, $\mathrm{cm}^{-1}$ ): 3055 (m), 2982 (m), 2773 (m), 1438 (s), 1111 (s); ${ }^{1} \mathrm{H}$ NMR $\left(\mathrm{CDCl}_{3}, 500.13 \mathrm{MHz}, \mathrm{ppm}\right): \delta 7.65-7.77$ (m, 9H, $\left.\mathrm{C}_{6} H_{5}\right), 7.56-7.63\left(\mathrm{~m}, 6 \mathrm{H}, \mathrm{C}_{6} H_{5}\right), 7.16-7.21\left(\mathrm{~m}, 1 \mathrm{H}, \mathrm{C}_{6} H_{5}\right), 7.04-7.10\left(\mathrm{~m}, 4 \mathrm{H}, \mathrm{C}_{6} H_{5}\right), 5.33$ $\left(\mathrm{d}, 2 \mathrm{H},{ }^{2} \mathrm{~J}_{\mathrm{H}, \mathrm{P}}=14.4 \mathrm{~Hz}, \mathrm{CH}_{2}\right) ;{ }^{13} \mathrm{C} \mathrm{NMR}\left(\mathrm{CDCl}_{3}, 125.76 \mathrm{MHz}, \mathrm{ppm}\right): \delta 135.08$ (phenyl-C), 133.44 (phenyl-C), 131.57 (phenyl-C), 130.23 (phenyl-C), 128.89 (phenyl-C), 128.47 (phenyl-C), 127.18 (phenyl-C), 117.82 (phenyl-C), $30.92\left(\mathrm{CH}_{2}\right) ;{ }^{31} \mathrm{P}\left\{{ }^{1} \mathrm{H}\right\} \mathrm{NMR}\left(\mathrm{CDCl}_{3}\right.$, 202.46 MHz, ppm): $\delta 23.15$ (s). 
Synthesis of triphenylphosphonium- $\alpha$-(trifluoroacetyl)benzylide (14). Benzyltriphenylphosphonium bromide $(\mathbf{1 3}, 500 \mathrm{mg}, 1.15 \mathrm{mmol})$ was dissolved under argon in anhydrous tetrahydrofuran $(10 \mathrm{~mL})$ and the white suspension cooled to $-10{ }^{\circ} \mathrm{C}$ using a mixture of ice and sodium chloride. Then, over a period of $45 \mathrm{~min}, 1.6 \mathrm{M} n$-butyllithium in $n$-hexane $(1.44 \mathrm{~mL}, 148 \mathrm{mg}, 2.31 \mathrm{mmol})$ was added, followed by stirring at $-10{ }^{\circ} \mathrm{C}$ for $1 \mathrm{~h}$. Afterwards, trifluoroacetic anhydride $(162 \mu \mathrm{L}, 242 \mathrm{mg}, 1.15 \mathrm{mmol})$ was added dropwise over a period of $10 \mathrm{~min}$ at $-10{ }^{\circ} \mathrm{C}$ and the mixture then stirred for $1 \mathrm{~h}$ at room temperature. After addition of isopropanol $(3 \mathrm{~mL})$, the solvent was removed under reduced pressure. The crude brown product was purified by column chromatography on silica using dichloromethane/methanol (49:1 v/v) as the eluent. The resulting yellow material was washed with diethyl ether $(10 \mathrm{~mL})$ to obtain a colorless solid. Yield: $20 \%$ (101 mg, $0.23 \mathrm{mmol}$ ). Elemental analysis (\%): calc. $\mathrm{C}_{27} \mathrm{H}_{20} \mathrm{~F}_{3} \mathrm{OP}$ : C 72.32, H 4.50, found: 72.44, H 4.60; IR (ATR, cm ${ }^{-1}$ ): 3056 (w), 1553 (s), 1159 (s), 1104 (s); ${ }^{1} \mathrm{H} \mathrm{NMR} \mathrm{(CDCl}{ }_{3}$, $500.13 \mathrm{MHz}, \mathrm{ppm}): \delta 7.56-7.49\left(\mathrm{~m}, 9 \mathrm{H}, \mathrm{PC}_{6} H_{5}\right), 7.43-7.37\left(\mathrm{~m}, 6 \mathrm{H}, \mathrm{PC}_{6} H_{5}\right), 7.05-6.97$ $\left(\mathrm{m}, 5 \mathrm{H}, \mathrm{CC}_{6} H_{5}\right) ;{ }^{13} \mathrm{C} \mathrm{NMR}\left(\mathrm{CDCl}_{3}, 125.76 \mathrm{MHz}, \mathrm{ppm}\right): \delta 170.39\left(\mathrm{dq},{ }^{2} J_{\mathrm{C}, \mathrm{F}}=30.1 \mathrm{~Hz}\right.$, $\left.{ }^{2} J_{\mathrm{C}, \mathrm{P}}=8.6 \mathrm{~Hz}, C=\mathrm{O}\right), 135.13\left(\mathrm{~d},{ }^{3} J_{\mathrm{C}, \mathrm{P}}=4.7 \mathrm{~Hz}\right.$, phenyl-C), $134.14\left(\mathrm{~d},{ }^{2} J_{\mathrm{C}, \mathrm{P}}=8.1 \mathrm{~Hz}\right.$, phenyl-C), 133.77 (d, ${ }^{3} J_{\mathrm{C}, \mathrm{P}}=9.7 \mathrm{~Hz}$, phenyl-C), 132.38 (d, ${ }^{4} J_{\mathrm{C}, \mathrm{P}}=2.4 \mathrm{~Hz}$, phenyl-C), $128.91\left(\mathrm{~d},{ }^{2} J_{\mathrm{C}, \mathrm{P}}=12.4 \mathrm{~Hz}\right.$, phenyl-C), $127.55\left(\mathrm{~d},{ }^{4} J_{\mathrm{C}, \mathrm{P}}=2.4 \mathrm{~Hz}\right.$, phenyl-C), 126.54 (d, ${ }^{5} J_{\mathrm{C}, \mathrm{P}}=3.0 \mathrm{~Hz}$, phenyl-C), $124.59\left(\mathrm{~d},{ }^{1} J_{\mathrm{C}, \mathrm{P}}=90.8 \mathrm{~Hz}, C-\mathrm{P}\right), 119.41\left(\mathrm{dq},{ }^{1} J_{\mathrm{C}, \mathrm{F}}=291.0 \mathrm{~Hz}\right.$, $\left.{ }^{3} J_{\mathrm{C}, \mathrm{P}}=20.1 \mathrm{~Hz}, C \mathrm{~F}_{3}\right)$, signal for $C=\mathrm{P}$ carbon atom observed; ${ }^{31} \mathrm{P}\left\{{ }^{1} \mathrm{H}\right\} \mathrm{NMR}\left(\mathrm{CDCl}_{3}\right.$, 202.46 MHz, ppm): $\delta 19.70\left(\mathrm{~s}, P \mathrm{Ph}_{3}\right) ;{ }^{19} \mathrm{~F} \mathrm{NMR}\left(\mathrm{CDCl}_{3}, 470.59 \mathrm{MHz}, \mathrm{ppm}\right): \delta-68.36(\mathrm{~d}$, $\left.{ }^{4} J_{\mathrm{F}, \mathrm{P}}=2.6 \mathrm{~Hz}, \mathrm{C} F_{3}\right)$.

Synthesis of phenyltrifluoromethylacetylene (15). A one-neck round-bottom flask filled with triphenylphosphonium- $\alpha$-(trifluoroacetyl)benzylide $(\mathbf{1 4}, 2.75 \mathrm{~g}, 6.14 \mathrm{mmol}$ ) was connected via a long glass tube to a Schlenk flask immersed in liquid dinitrogen, which was heated to $300{ }^{\circ} \mathrm{C}$ under vacuum $\left(3 \cdot 10^{-1}\right.$ mbar) for $2 \mathrm{~h}$ with a heat gun. The collection flask was allowed to reach room temperature and the product collected as a clear colorless liquid. Yield: $71 \%$ (740 mg, $4.35 \mathrm{mmol})$. Elemental analysis (\%): calc. $\mathrm{C}_{9} \mathrm{H}_{5} \mathrm{~F}_{3}$ : C 63.54, H 2.96, found: C 63.39, H 2.97; IR (ATR, $\mathrm{cm}^{-1}$ ): 2254 (s), 1493 (m), 1309 (vs), 1124 (vs); ${ }^{1} \mathrm{H}$ NMR $\left(\mathrm{CDCl}_{3}, 400.40 \mathrm{MHz}, \mathrm{ppm}\right): \delta 7.60-7.54\left(\mathrm{~m}, 2 \mathrm{H}, \mathrm{C}_{6} H_{5}\right), 7.51-7.45(\mathrm{~m}, 1 \mathrm{H}$, $\left.\mathrm{C}_{6} H_{5}\right), 7.44-7.37\left(\mathrm{~m}, 2 \mathrm{H}, \mathrm{C}_{6} H_{5}\right) ;{ }^{13} \mathrm{C} \mathrm{NMR}\left(\mathrm{CDCl}_{3}, 100.68 \mathrm{MHz}, \mathrm{ppm}\right): \delta 132.59\left(\mathrm{q},{ }^{5} J_{\mathrm{C}, \mathrm{F}}\right.$ $=1.6 \mathrm{~Hz}$, phenyl-C), $131.02\left(\mathrm{~s}\right.$, phenyl-C), 128.80 (s, phenyl-C), $118.68\left(\mathrm{q},{ }^{4} J_{\mathrm{C}, \mathrm{F}}=1.8 \mathrm{~Hz}\right.$, $\left.C \equiv \mathrm{C}-\mathrm{CF}_{3}\right), 115.00\left(\mathrm{q},{ }^{1} J_{\mathrm{C}, \mathrm{F}}=257.0 \mathrm{~Hz}, C \mathrm{~F}_{3}\right), 86.67\left(\mathrm{q},{ }^{3} J_{\mathrm{C}, \mathrm{F}}=6.5 \mathrm{~Hz}\right.$, phenyl-C), 75.85 $\left(\mathrm{q},{ }^{2} J_{\mathrm{C}, \mathrm{F}}=52.5 \mathrm{~Hz}, \mathrm{C} \equiv C-\mathrm{CF}_{3}\right) ;{ }^{19} \mathrm{~F} \mathrm{NMR}\left(\mathrm{CDCl}_{3}, 376.75 \mathrm{MHz}, \mathrm{ppm}\right): \delta-49.80\left(\mathrm{~s}, \mathrm{CF}_{3}\right)$. 
Synthesis of [W(bpy)(CO) $)_{4}$ (7). Tungsten hexacarbonyl (1.00 g, $\left.2.84 \mathrm{mmol}\right)$ was suspended under argon in degassed benzene $(25 \mathrm{~mL})$. Then, under efficient stirring, a solution of trimethylamine- $N$-oxide dihydrate (TMAO, $679 \mathrm{mg}, 6.11 \mathrm{mmol}$ ) in degassed methanol $(10 \mathrm{~mL})$ was added, which resulted in the solution to turn yellow immediately. Then, solid 2,2'-bipyridine (470 mg, $3.01 \mathrm{mmol}$ ) was added, upon which the solution turned red over the course of $1 \mathrm{~h}$. Stirring was continued at room temperature for $2 \mathrm{~d}$. The resulting red-brown solid which had precipitated was filtered off and recrystallized from acetonitrile $(100 \mathrm{~mL})$. Finally, the product was washed with $n$-hexane $(50 \mathrm{~mL})$ and dried under vacuum overnight, resulting in a dark violet crystalline solid. Yield: 31\% (399 mg, $0.88 \mathrm{mmol}$ ). Elemental analysis (\%): calc. $\mathrm{C}_{14} \mathrm{H}_{8} \mathrm{~N}_{2} \mathrm{O}_{4} \mathrm{~W}$ : C 37.20, H 1.78, N 6.20, found: C 37.16, H 1.96, N 6.17; IR (ATR, $\mathrm{cm}^{-1}$ ): 2001 (m), 1902 (m), 1821 (s), 1787 (s), 1599 (s), 1467 (s), 1437 (m), 759 (m); ${ }^{1} \mathrm{H}$ NMR (DMSO- $\left.d_{6}, 500.13 \mathrm{MHz}, \mathrm{ppm}\right): \delta 9.09$ (ddd, $2 \mathrm{H},{ }^{3} J_{\mathrm{H} 6, \mathrm{H} 5}=5.5 \mathrm{~Hz},{ }^{4} J_{\mathrm{H} 6, \mathrm{H} 4}=$ $1.6 \mathrm{~Hz},{ }^{5} J_{\mathrm{H} 6, \mathrm{H} 3}=0.8 \mathrm{~Hz}$, bpy-H6), $8.71\left(\mathrm{dt}, 2 \mathrm{H},{ }^{3} J_{\mathrm{H} 3, \mathrm{H} 4}=8.3 \mathrm{~Hz},{ }^{4} J_{\mathrm{H} 3, \mathrm{H} 5}=1.0 \mathrm{~Hz}\right.$, bpy$\mathrm{H} 3), 8.24\left(\mathrm{ddd}, 2 \mathrm{H},{ }^{3} J_{\mathrm{H} 4, \mathrm{H} 3}=8.1 \mathrm{~Hz},{ }^{3} J_{\mathrm{H} 4, \mathrm{H} 5}=7.6 \mathrm{~Hz},{ }^{4} J_{\mathrm{H} 4, \mathrm{H} 6}=1.6 \mathrm{~Hz}\right.$, bpy-H4), 7.67 $\left(\mathrm{ddd}, 2 \mathrm{H},{ }^{3} J_{\mathrm{H} 5, \mathrm{H} 4}=7.5 \mathrm{~Hz},{ }^{3} J_{\mathrm{H} 5, \mathrm{H} 6}=5.5 \mathrm{~Hz},{ }^{4} J_{\mathrm{H} 5, \mathrm{H} 3}=1.3 \mathrm{~Hz}\right.$, bpy-H5); ${ }^{13} \mathrm{C} \mathrm{NMR}$ (DMSO- $\left.d_{6}, 125.76 \mathrm{MHz}, \mathrm{ppm}\right): \delta 214.80(\mathrm{C} \equiv \mathrm{O}), 201.64(\mathrm{C} \equiv \mathrm{O}), 155.22$ (bpy-C2), 152.41 (bpy-C6), 138.84 (bpy-C4), 127.04 (bpy-C5), 124.05 (bpy-C3).

Synthesis of $\left[\mathbf{W}(\mathbf{b p y})(\mathbf{C O})_{\mathbf{3}}(\mathbf{p y})\right]$ (8). Pyridine $(1 \mathrm{~mL}, 0.98 \mathrm{~g}, 12.4 \mathrm{mmol})$ was dissolved in degassed xylene $(10 \mathrm{~mL})$, solid $\left[\mathrm{W}(\mathrm{bpy})(\mathrm{CO})_{4}\right](7,250 \mathrm{mg}, 0.55 \mathrm{mmol})$ added, and the reaction mixture heated to reflux for $18 \mathrm{~h}$. The resulting black precipitate was filtered from the yellow-red solution, washed with toluene $(50 \mathrm{~mL})$ and methanol $(20 \mathrm{~mL})$ and dried under vacuum overnight. Yield: 76\% (213 mg, 0.42 mmol). Elemental analysis (\%): calc. $\mathrm{C}_{18} \mathrm{H}_{13} \mathrm{~N}_{3} \mathrm{O}_{3} \mathrm{~W}: \mathrm{C} 42.97, \mathrm{H} 2.60, \mathrm{~N} 8.35$, found: $\mathrm{C}$ 40.37, H 2.70, N 7.40; IR (ATR, cm ${ }^{-1}$ ): 1874 (s) 1745 (s), 1600 (w), 1466 (w), 1440 (m), $763(\mathrm{~m})$; since the pyridine ligand rapidly exchanged with solvent, for example in DMSO, no NMR assignment was made. 


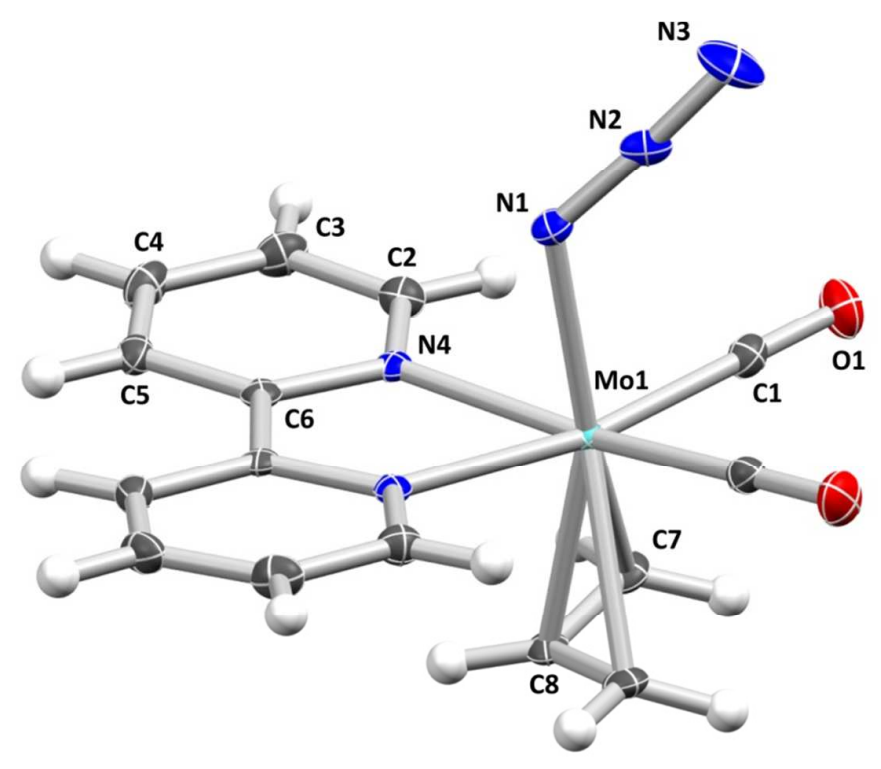

Fig. S1 Molecular structure of $\left[\mathrm{Mo}\left(\eta^{3}\right.\right.$-allyl $\left.)\left(\mathrm{N}_{3}\right)(\mathrm{bpy})(\mathrm{CO})_{2}\right]$ (2) with thermal ellipsoids displayed at the $50 \%$ probability level. The asymmetric unit contains only one half of the molecule with the other one related to it by a mirror plane. Selected bond lengths $[\AA]$ and angles $\left[{ }^{\circ}\right.$ ] for 2: Mo1-C1 1.956(2), Mo1-N1 2.180(3), Mo1-N4 2.240(2), Mo1-(allyl centroid) 2.044(2), C1-O1 1.163(3), C7-C8 1.409(3), N1-N2 1.201(4), N2-N3 1.152(4), C1Mo1-C1a 81.4(1), N4-Mo1-N4a 72.3(1), C1-Mo1-N4 102.53(9), N1-Mo1-(allyl centroid) 174.24(9), C7-C8-C7a 114.8(3), Mo1-N1-N2 124.9(2), N1-N2-N3 176.7(4). 


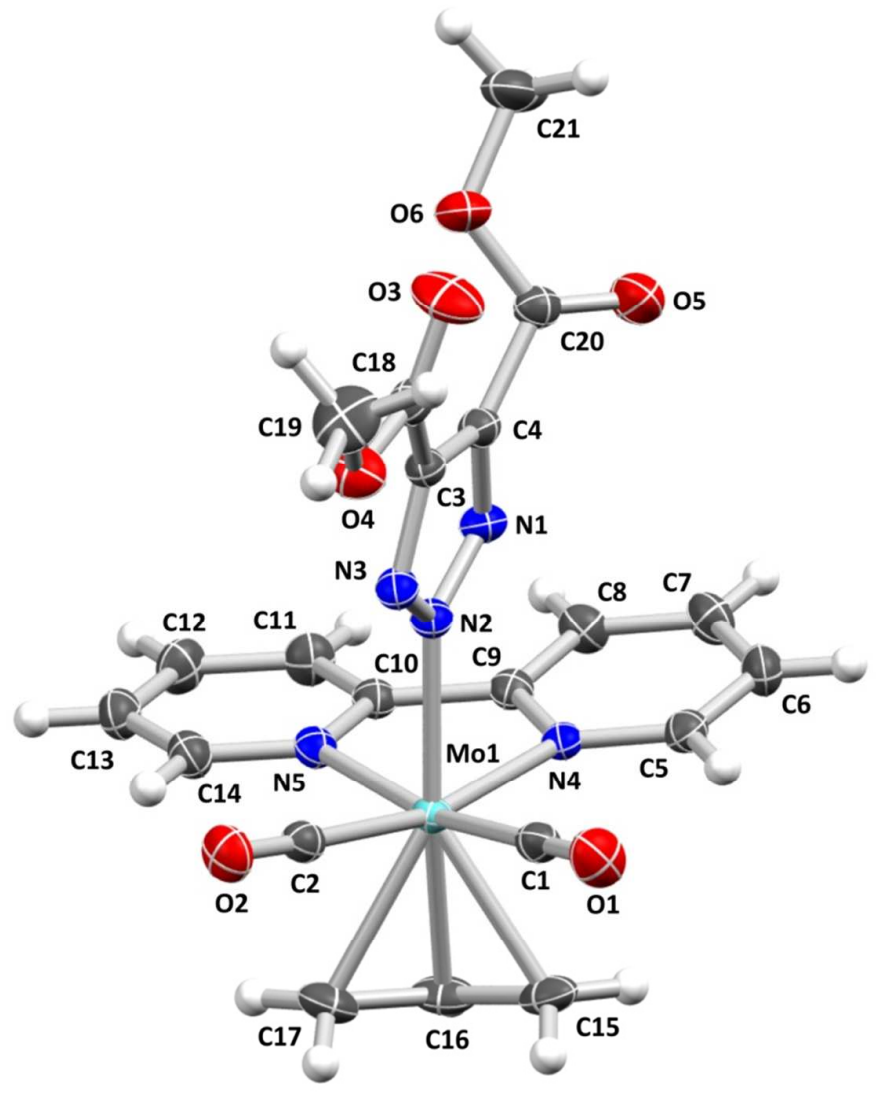

Fig. S2 Molecular structure of $\left[\mathrm{Mo}\left(\eta^{3}\right.\right.$-allyl $)\left(\right.$ triazolate $\left.\left.^{\mathrm{COOCH} 3, \mathrm{COOCH} 3}\right)(\mathrm{bpy})(\mathrm{CO})_{2}\right]$ (5) with thermal ellipsoids displayed at the $50 \%$ probability level. Selected bond lengths $[\AA]$ and angles [ ${ }^{\circ}$ ] for 5: Mo1-C1 1.978(2), Mo1-C2 1.969(2), Mo1-N2 2.221(2), Mo1-N4 2.255(2), Mo1-N5 2.258(2), Mo1-(allyl centroid) 2.056(2), C1-O1 1.156(3), C2-O2 1.160(3), C15C16 1.408(4), C16-C17 1.411(3), C1-Mo1-C2 80.47(9), N4-Mo1-N5 72.77(7), C1-Mo1N4 104.38(8), C2-Mo1-N5 100.25(8), N2-Mo1-(allyl centroid) 177.63(6), C15-C16-C17 116.1(2), (N2-Mo1-C16)-(triazolate) 13.6(3). 


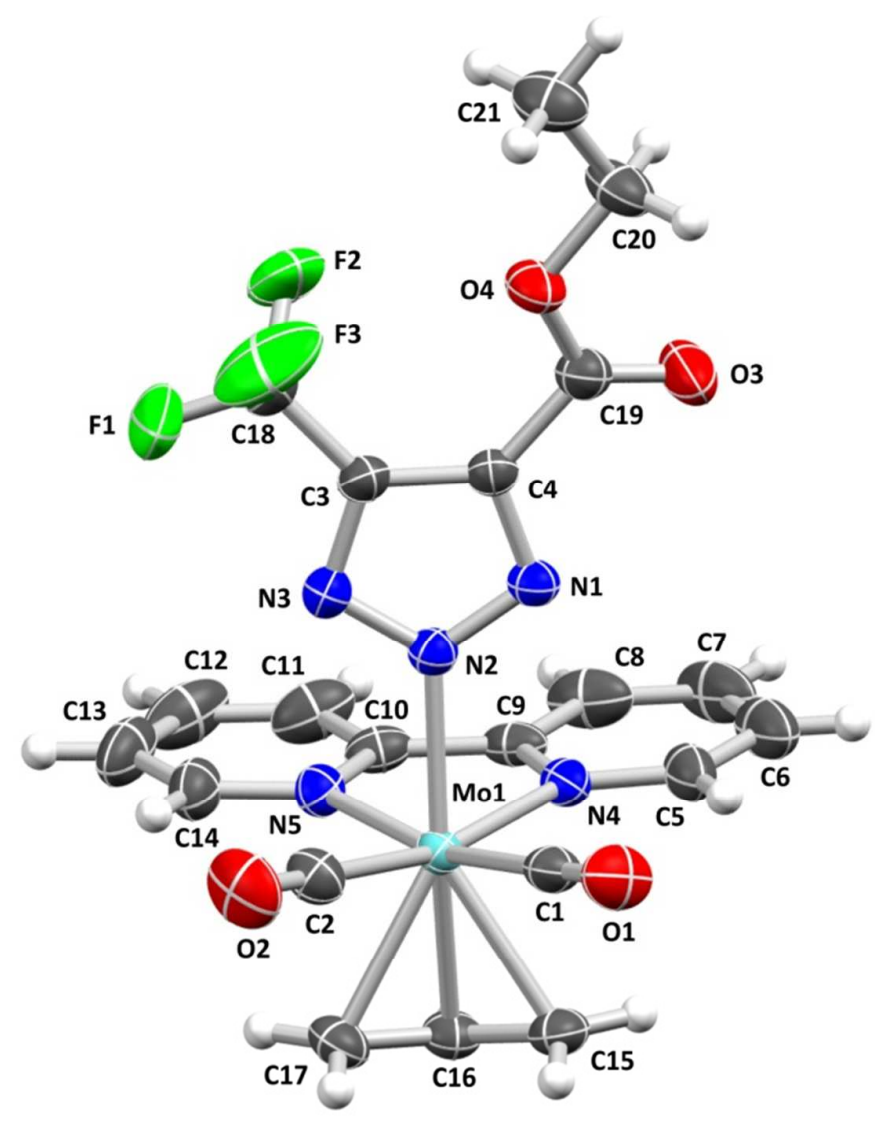

Fig. S3 Molecular structure of $\left[\mathrm{Mo}\left(\eta^{3}\right.\right.$-allyl $)\left(\right.$ triazolate $\left.\left.{ }^{\mathrm{CF} 3, \mathrm{COOEt}}\right)(\mathrm{bpy})(\mathrm{CO})_{2}\right](6)$ with thermal ellipsoids displayed at the $50 \%$ probability level. Selected bond lengths $[\AA]$ and angles $\left[{ }^{\circ}\right]$ for 6: Mo1-C1 1.954(3), Mo1-C2 1.964(2), Mo1-N2 2.210(2), Mo1-N4 2.218(2), Mo1-N5 2.234(2), Mo1-(allyl centroid) 2.048(2), C1-O1 1.156(3), C2-O2 1.150(3), C15-C16 1.400(4), C16-C17 1.396(4), C1-Mo1-C2 81.4(1), N4-Mo1-N5 73.11(8), C1-Mo1-N4 101.8(1), C2-Mo1-N5 101.4(1), N2-Mo1-(allyl centroid) 176.37(7), C15-C16-C17 116.4(3), (N2-Mo1-C16)-(triazolate) 80.4(3). 


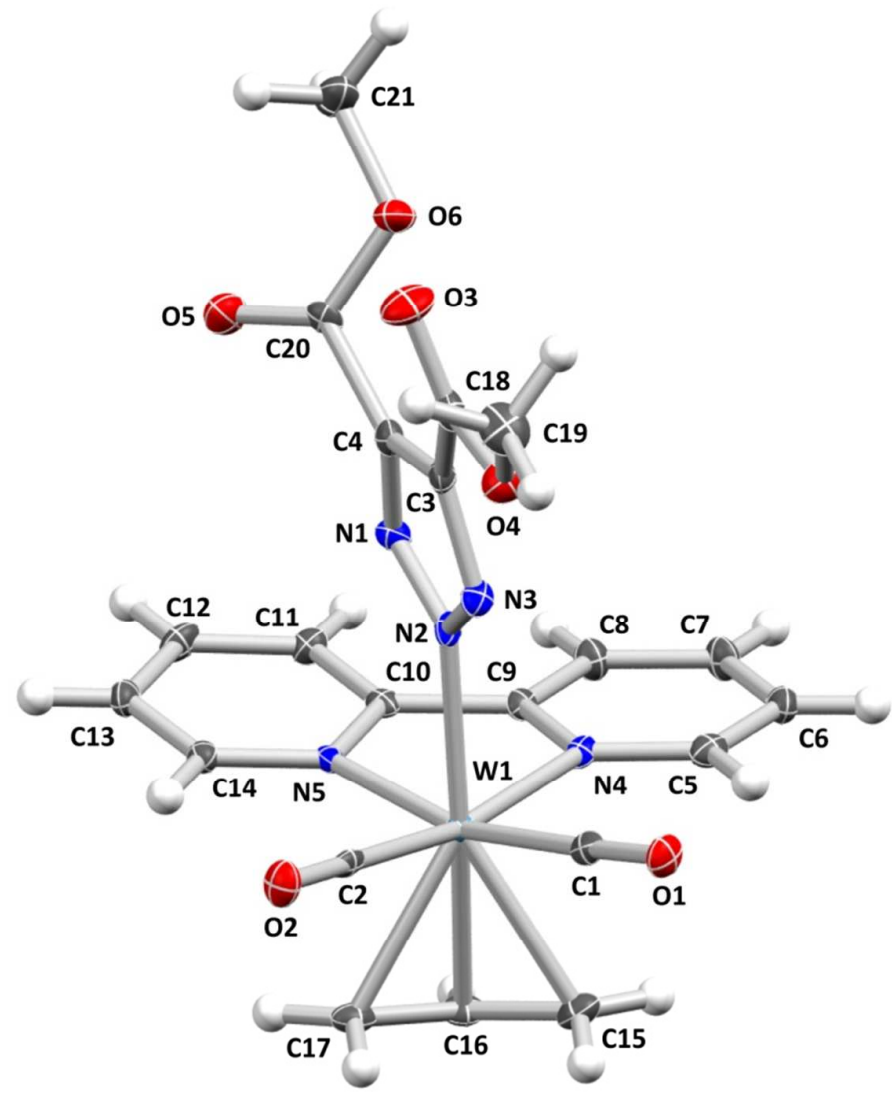

Fig. S4 Molecular structure of $\left[\mathrm{W}\left(\eta^{3}\right.\right.$-allyl $)\left(\right.$ triazolate $\left.\left.^{\mathrm{COOCH} 3, \mathrm{COOCH} 3}\right)(\mathrm{bpy})(\mathrm{CO})_{2}\right](\mathbf{1 1})$ with thermal ellipsoids displayed at the $50 \%$ probability level. Selected bond lengths $[\AA]$ and angles [ $\left.{ }^{\circ}\right]$ for 11: W1-C1 1.957(4), W1-C2 1.960(4), W1-N2 2.194(3), W1-N4 2.224(3), W1-N5 2.231(3), W1-(allyl centroid) 2.032(2), C1-O1 1.163(4), C2-O2 1.165(5), C15-C16 1.402(6), C16-C17 1.404(6), C1-W1-C2 80.9(2), N4-W1-N5 73.1(1), C1-W1-N4 99.7(1), C2-W1-N5 104.1(1), N2-W1-(allyl centroid) 177.6(1), C15-C16-C17 115.8(3), (N2-W1C16)-(triazolate) 15.6(5). 
Table S1 Crystallographic parameters for $\mathbf{2 , 5}$, and $\mathbf{6}$ as well as $\mathbf{1 0 - 1 2}$

\begin{tabular}{|c|c|c|c|c|c|c|}
\hline Compound & 2 & 5 & 6 & 10 & 11 & 12 \\
\hline Empirical formula & $\mathrm{C}_{15} \mathrm{H}_{13} \mathrm{MoN}_{5} \mathrm{O}_{2}$ & $\mathrm{C}_{21} \mathrm{H}_{19} \mathrm{MoN}_{5} \mathrm{O}_{6}$ & $\mathrm{C}_{21} \mathrm{H}_{18} \mathrm{~F}_{3} \mathrm{MoN}_{5} \mathrm{O}_{4}$ & $\mathrm{C}_{15} \mathrm{H}_{13} \mathrm{~N}_{5} \mathrm{O}_{2} \mathrm{~W}$ & $\mathrm{C}_{21} \mathrm{H}_{19} \mathrm{~N}_{5} \mathrm{O}_{6} \mathrm{~W}$ & $\mathrm{C}_{21} \mathrm{H}_{18} \mathrm{~F}_{3} \mathrm{~N}_{5} \mathrm{O}_{4} \mathrm{~W}$ \\
\hline Formula weight $\left(\mathrm{g} \cdot \mathrm{mol}^{-1}\right)$ & 391.24 & 533.35 & 557.34 & 479.15 & 621.26 & 645.25 \\
\hline Temperature (K) & $100(2)$ & 173(2) & 273(2) & $100(2)$ & $100(2)$ & $100(2)$ \\
\hline Radiation, $\lambda(\AA)$ & $\mathrm{Mo}_{\mathrm{K} \alpha} 0.71073$ & $\mathrm{Mo}_{\mathrm{K} \alpha} 0.71073$ & $\mathrm{Mo}_{\mathrm{K \alpha}} 0.71073$ & $\mathrm{Mo}_{\mathrm{K} \alpha} 0.71073$ & $\mathrm{Mo}_{\mathrm{K} \alpha} 0.71073$ & $\mathrm{Mo}_{\mathrm{K \alpha}} 0.71073$ \\
\hline Crystal system & Orthorhombic & Monoclinic & Triclinic & Orthorhombic & Monoclinic & Monoclinic \\
\hline Space group & Pnma & $P 2_{1} / c$ & $P \overline{1}$ & Pnma & $P 2_{1} / c$ & $P 2_{1} / c$ \\
\hline \multicolumn{7}{|l|}{ Unit cell dimensions } \\
\hline$a(\AA)$ & $16.96(1)$ & $11.815(8)$ & $10.545(2)$ & $16.83(3)$ & $11.697(6)$ & $11.078(5)$ \\
\hline$b(\AA)$ & $13.755(6)$ & $9.369(6)$ & $10.851(2)$ & $13.77(2)$ & $9.262(6)$ & $9.984(5)$ \\
\hline$c(\AA)$ & $6.589(3)$ & $20.13(1)$ & $11.320(2)$ & $6.602(8)$ & $19.89(1)$ & $19.17(1)$ \\
\hline$\alpha\left(^{\circ}\right)$ & 90 & 90 & $82.745(7)$ & 90 & 90 & 90 \\
\hline$\beta\left(^{\circ}\right)$ & 90 & $103.28(1)$ & $63.061(8)$ & 90 & $103.39(2)$ & $91.10(2)$ \\
\hline$\gamma\left({ }^{\circ}\right)$ & 90 & 90 & $75.89(2)$ & 90 & 90 & 90 \\
\hline Volume $\left(\AA^{3}\right)$ & $1537(1)$ & 2169(3) & $1119.7(4)$ & 1530(4) & 2096(2) & $2120(2)$ \\
\hline$Z$ & 4 & 4 & 2 & 4 & 4 & 4 \\
\hline Calculated density $\left(\mathrm{kg} \cdot \mathrm{m}^{-3}\right)$ & 1.690 & 1.633 & 1.653 & 2.080 & 1.969 & 2.022 \\
\hline Absorption coefficient $\left(\mathrm{mm}^{-1}\right)$ & 0.870 & 0.654 & 0.649 & 7.566 & 5.563 & 5.517 \\
\hline$F(000)$ & 784 & 1080 & 560 & 912 & 1208 & 1248 \\
\hline Theta range for collection $\left(2 \Theta,^{\circ}\right)$ & 2.822 to 26.019 & 1.771 to 26.019 & 2.216 to 26.843 & 2.837 to 28.279 & 2.438 to 26.022 & 1.839 to 26.022 \\
\hline Reflections collected & 9005 & 17803 & 15715 & 9425 & 15002 & 24833 \\
\hline Independent reflections & 1577 & 4262 & 4788 & 1979 & 4133 & 4178 \\
\hline $\begin{array}{l}\text { Minimum/maximum transmission } \\
\text { Refinement method }\end{array}$ & $\underset{\mathrm{a}}{0.6445 / 0.7465}$ & $\underset{\mathrm{a}}{0.5979 / 0.7453}$ & $\underset{\mathrm{a}}{0.8842 / 0.9763}$ & $0.5011 / \mathrm{a} 0.7471$ & $\underset{\mathrm{a}}{0.4438 / 0.7465}$ & $\underset{\mathrm{a}}{0.5169 / 0.7471}$ \\
\hline Data / parameters / restraints & 1577 / $120 / 0$ & $4262 / 300 / 0$ & $4788 / 325 / 5$ & $1979 / 120 / 0$ & $4133 / 315 / 5$ & $4178 / 323 / 5$ \\
\hline Goodness-of-fit on $F^{2}$ & 1.035 & 1.060 & 1.034 & 1.020 & 1.026 & 1.049 \\
\hline Final $R$ indices $[I>2 \sigma(I)]$ & $\begin{array}{l}\mathrm{R}_{1}=0.0237 \\
w \mathrm{R}_{2}=0.0555\end{array}$ & $\mathrm{R}_{1}=0.0260$ & $\mathrm{R}_{1}=0.0330$ & $\mathrm{R}_{1}=0.0220$ & $\mathrm{R}_{1}=0.0227$ & $\mathrm{R}_{1}=0.0155$ \\
\hline$R$ indices (all data) & $\begin{array}{c}w \mathrm{R}_{2}=0.0555 \\
\mathrm{R}_{1}=0.0332\end{array}$ & $\begin{array}{c}w \mathrm{R}_{2}=0.0656 \\
\mathrm{R}_{1}=0.0292\end{array}$ & $\begin{array}{c}w \mathrm{R}_{2}=0.0634 \\
\mathrm{R}_{1}=0.0476\end{array}$ & $\begin{array}{l}w \mathrm{R}_{2}=0.0452 \\
\mathrm{R}_{1}=0.0352\end{array}$ & $\begin{array}{c}w \mathrm{R}_{2}=0.0487 \\
\mathrm{R}_{1}=0.0299\end{array}$ & $\begin{array}{c}w \mathrm{R}_{2}=0.0345 \\
\mathrm{R}_{1}=0.0172\end{array}$ \\
\hline & $w \mathrm{R}_{2}=0.0596$ & $w \mathrm{R}_{2}=0.0676$ & $w \mathrm{R}_{2}=0.0679$ & $w \mathrm{R}_{2}=0.0501$ & $w \mathrm{R}_{2}=0.0511$ & $w \mathrm{R}_{2}=0.0351$ \\
\hline $\begin{array}{l}\text { Maximum/minimum residual } \\
\text { electron density }\left(e \cdot \AA^{-3}\right)\end{array}$ & $0.512 /-0.461$ & $0.330 /-0.292$ & $0.368 /-0.407$ & $1.380 /-0764$ & $0.787 /-0.889$ & $0.873 /-0.626$ \\
\hline
\end{tabular}

${ }^{\mathrm{a}}$ Full-matrix least-squares on $F^{2}$. 


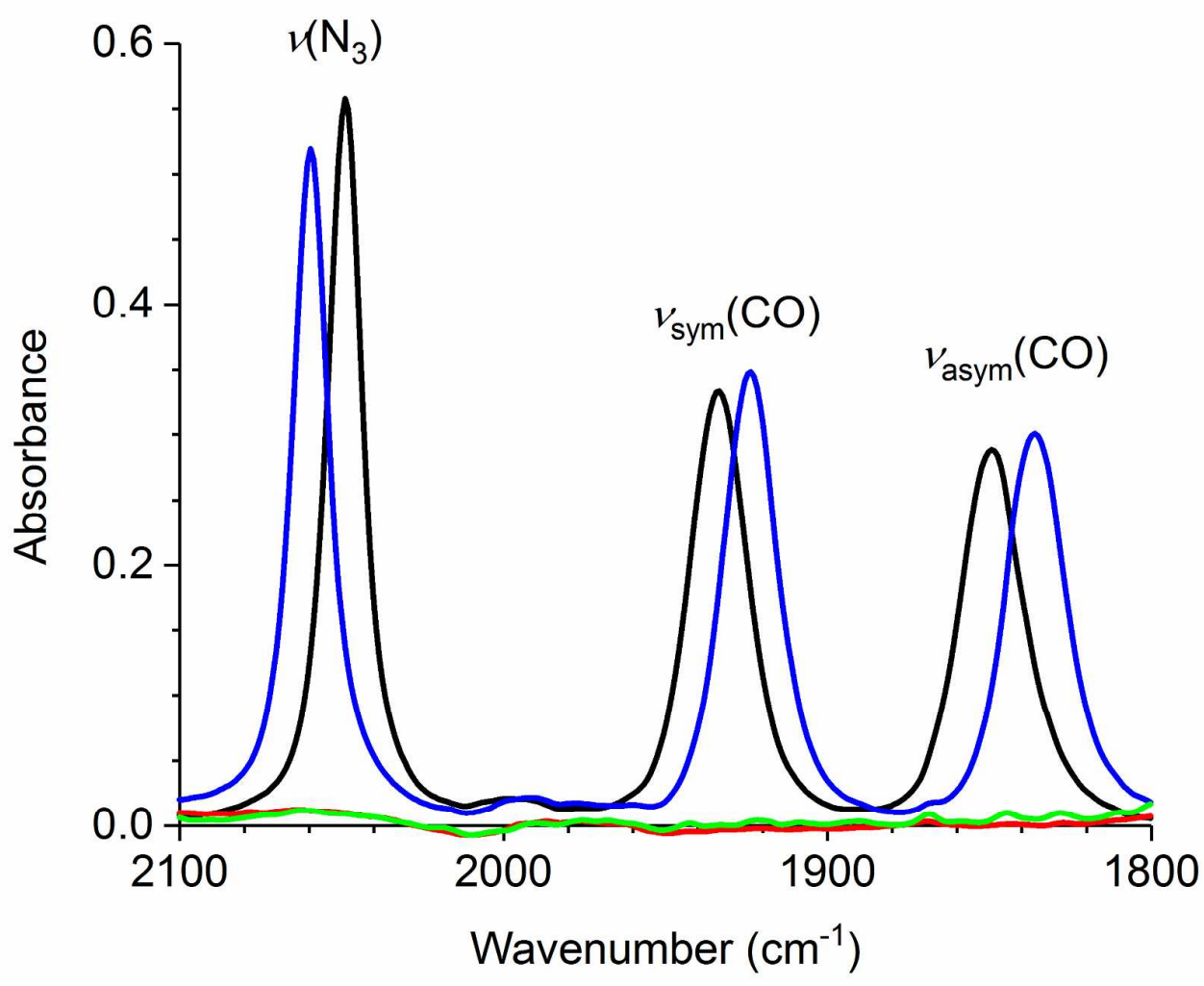

Fig. S5 Solution IR spectra of azide complexes 2 (black, $8 \mathrm{mM}$ ) and $\mathbf{1 0}$ (blue, $8 \mathrm{mM}$ ) as well as alkynes 3 (red, $40 \mathrm{mM}$ ) and $\mathbf{4}$ (green, $40 \mathrm{mM}$ ) in dimethylsulfoxide. 

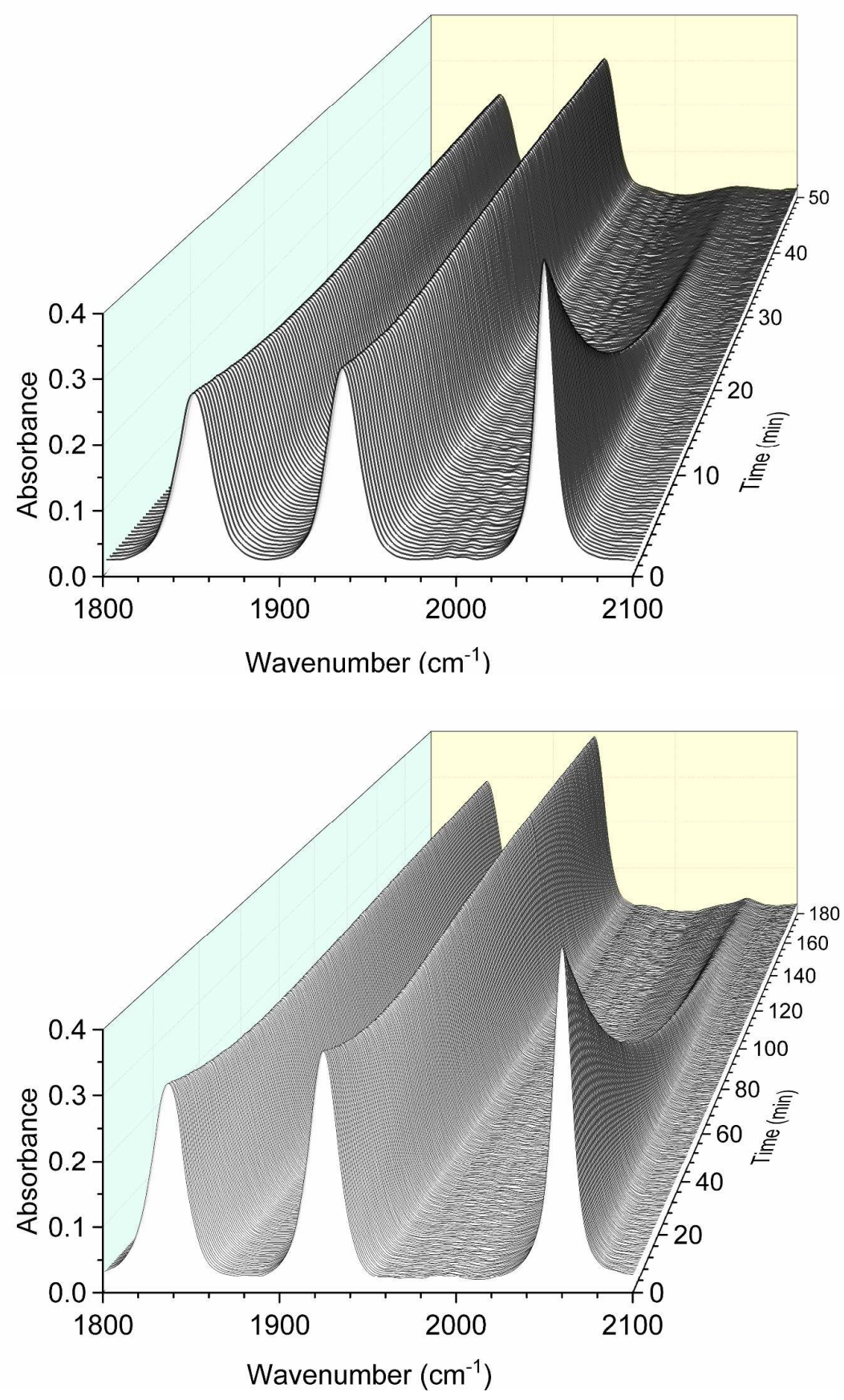

Fig. S6 Changes in the $1800-2100 \mathrm{~cm}^{-1}$ spectral range of the IR spectrum of a mixture of $\mathbf{2}$ (top) or $\mathbf{1 0}$ (bottom) in dimethylsulfoxide $(8 \mathrm{mM})$ upon reaction with dimethyl acetylenedicarboxylate (DMAD, 3, $40 \mathrm{mM}$ ) at $26^{\circ} \mathrm{C}$ in $20 \mathrm{~s}$ intervals for up to $180 \mathrm{~min}$. 


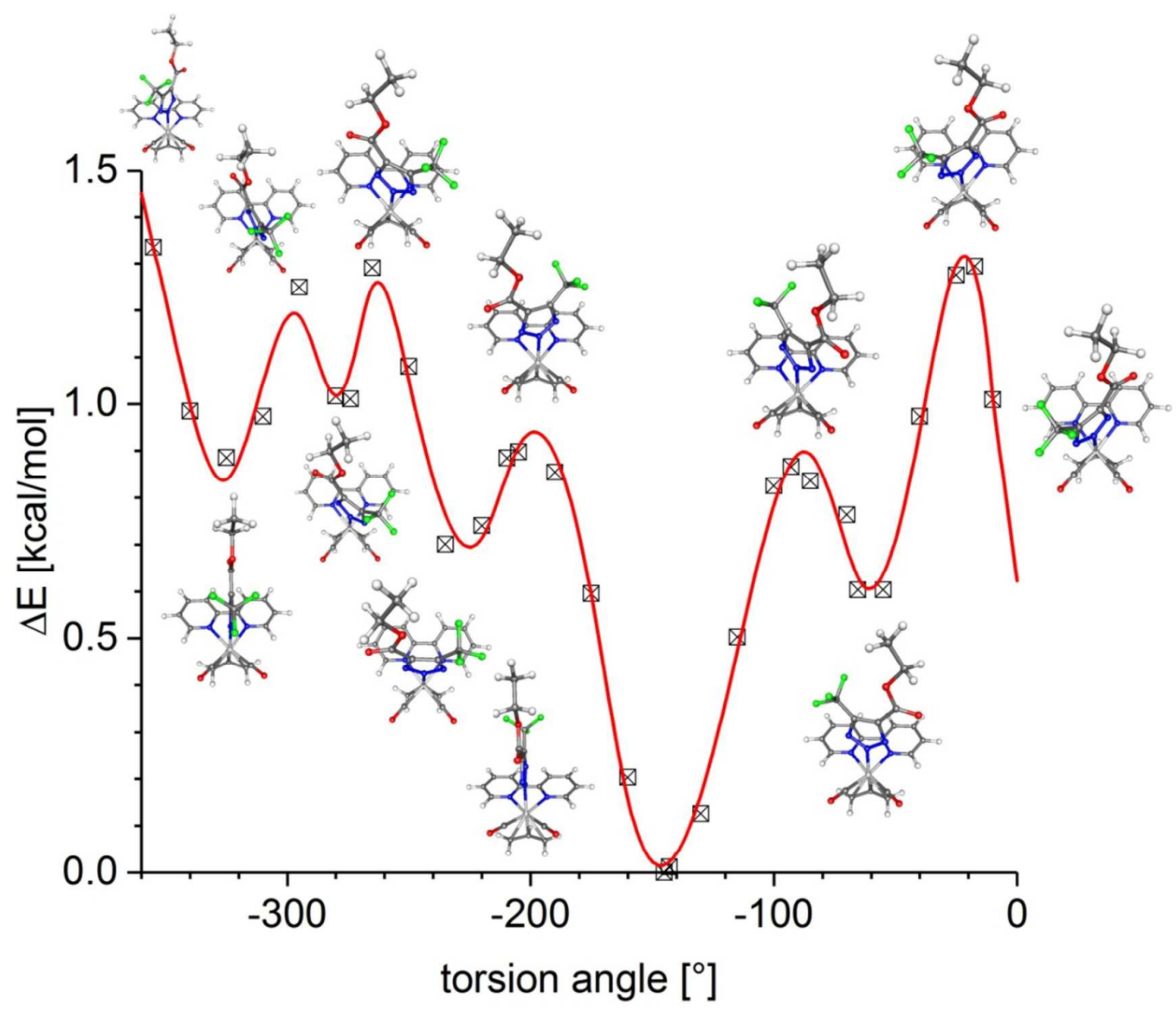

Fig. S7 Conformational energy diagram of 6 for variation of the torsion angle between the triazolate mean plane and the bpy $\mathrm{C} 2-\mathrm{C} 2$ ' axis calculated with DFT (ORCA 2.8, BP86, RI, TZVP, COSMO in DMSO). 
A)

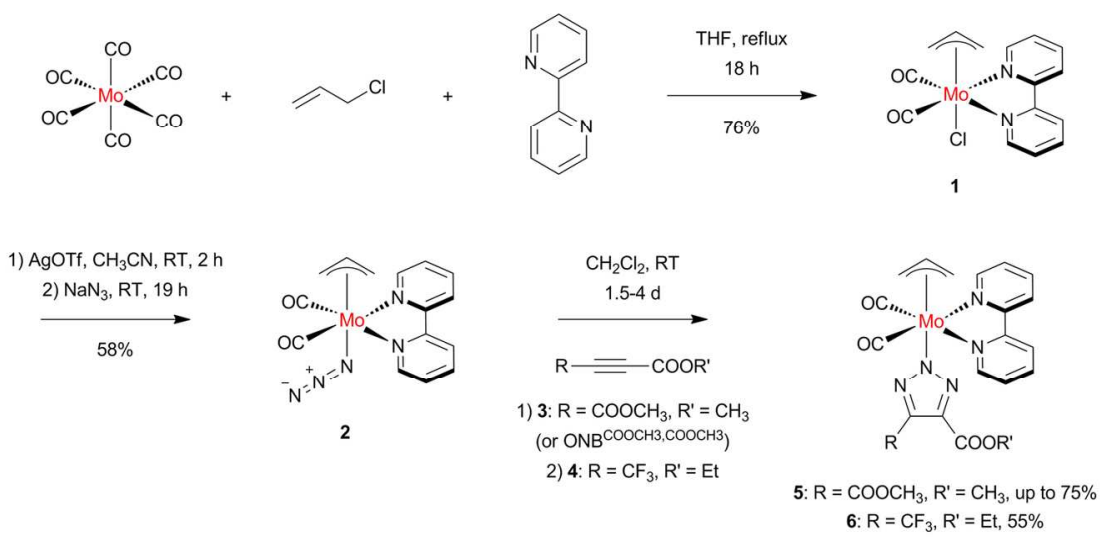

B)
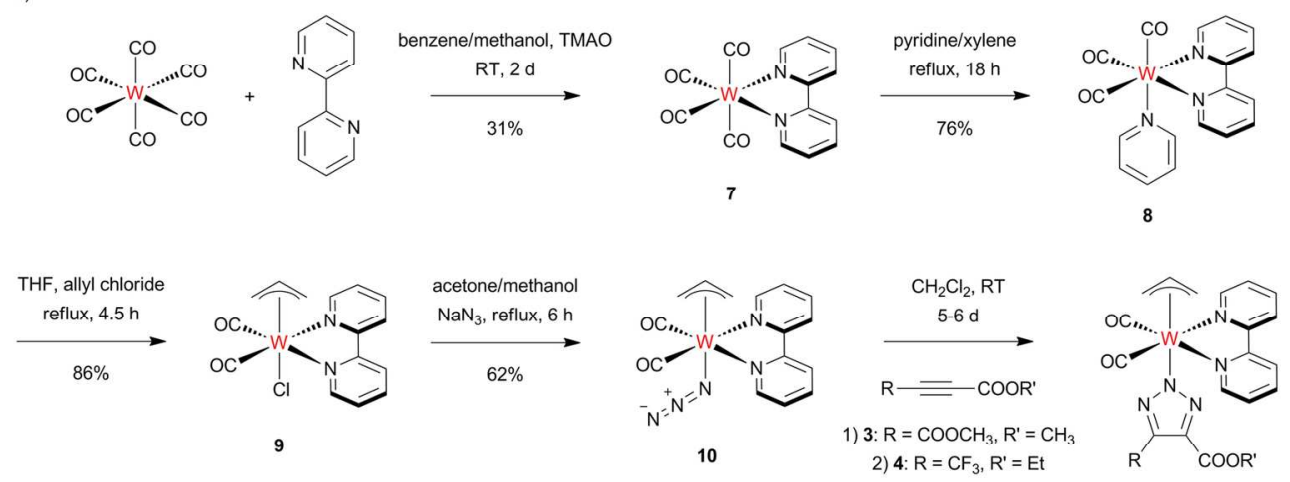

11: $\mathrm{R}=\mathrm{COOCH}_{3}, \mathrm{R}^{\prime}=\mathrm{CH}_{3}, 83 \%$ 12: $\mathrm{R}=\mathrm{CF}_{3}, \mathrm{R}^{\prime}=\mathrm{Et}, 40 \%$

$152 \times 132 \mathrm{~mm}(300 \times 300 \mathrm{DPI})$ 


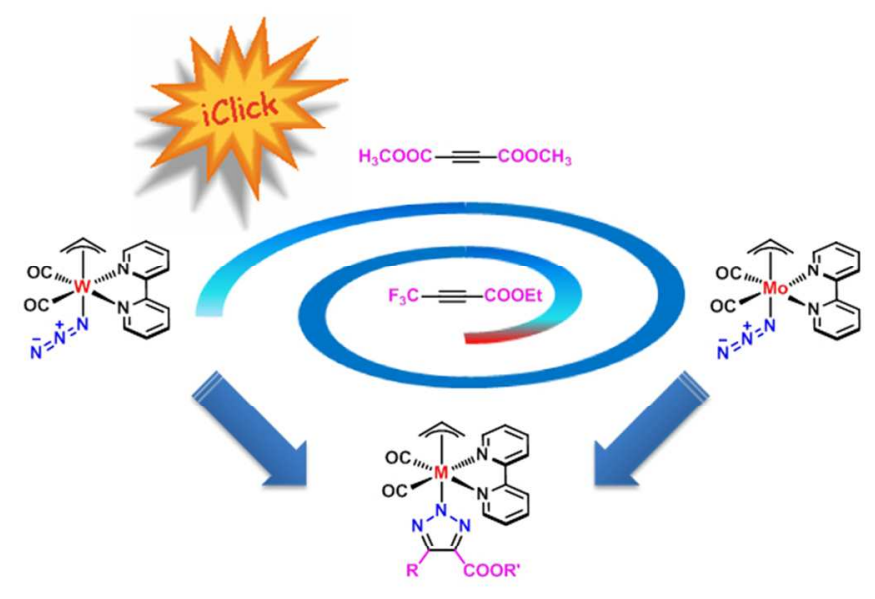

$254 \times 190 \mathrm{~mm}(96 \times 96$ DPI) 


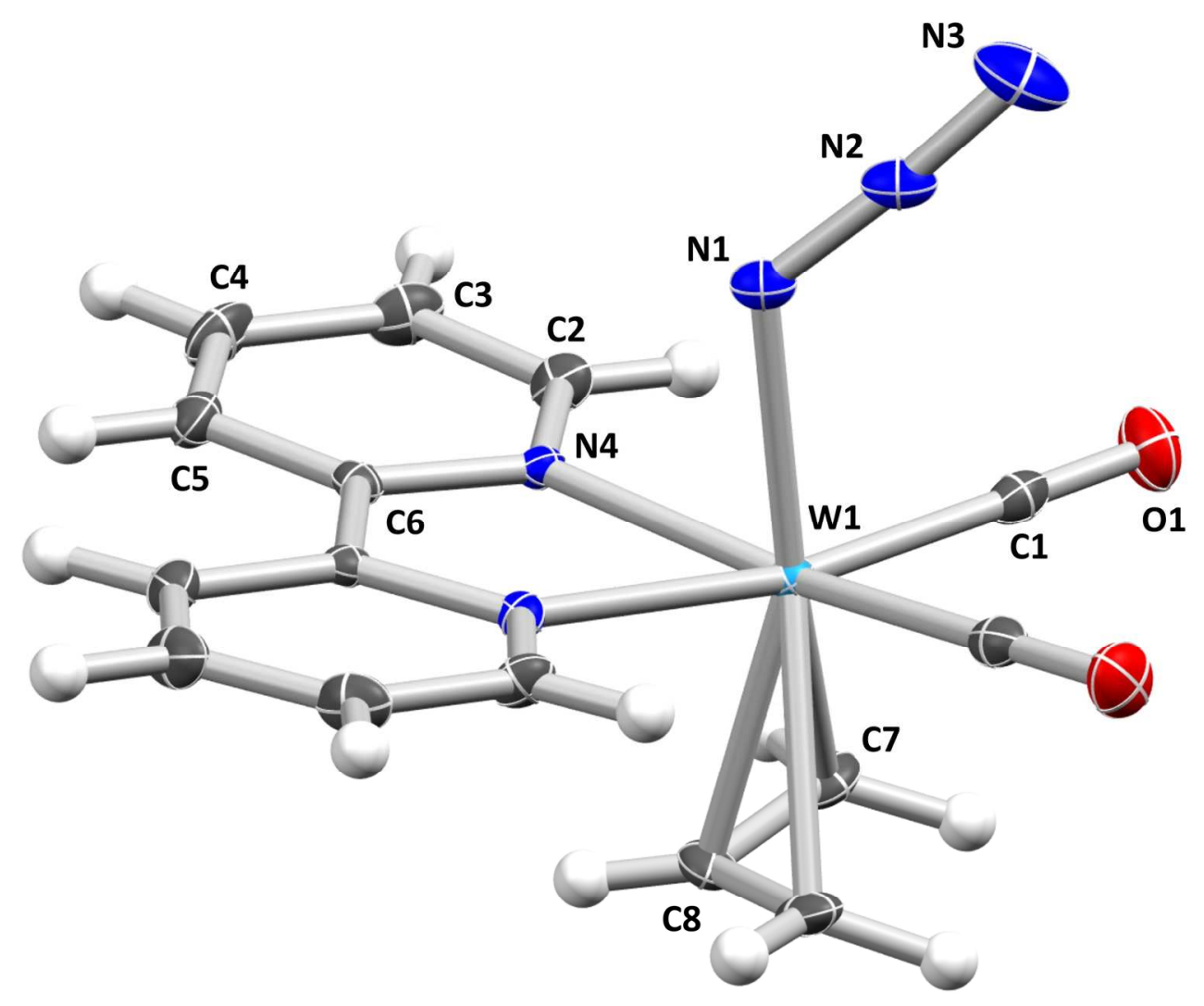

$165 \times 136 \mathrm{~mm}(300 \times 300 \mathrm{DPI})$ 


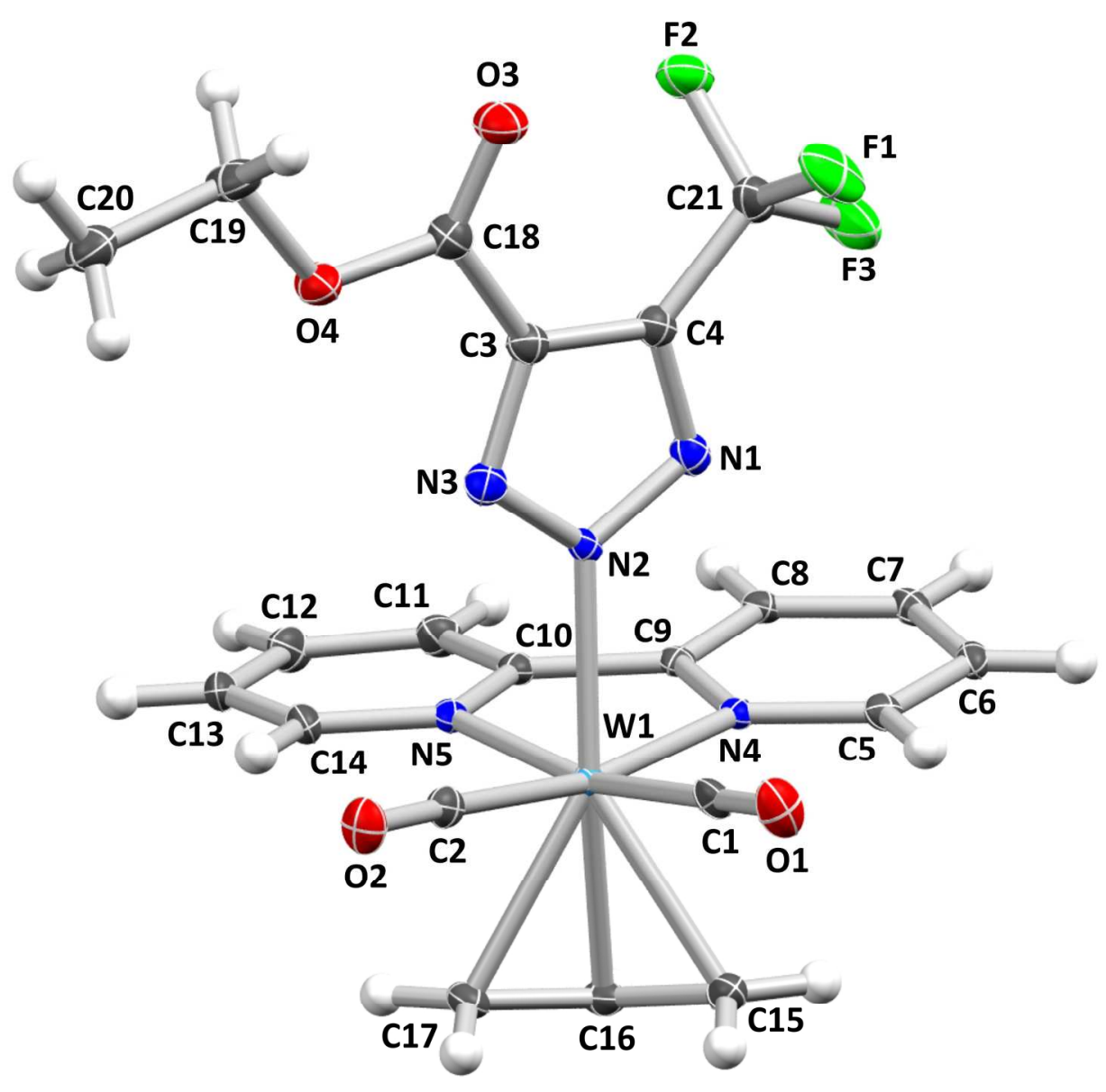

$187 \times 176 \mathrm{~mm}(300 \times 300$ DPI $)$ 


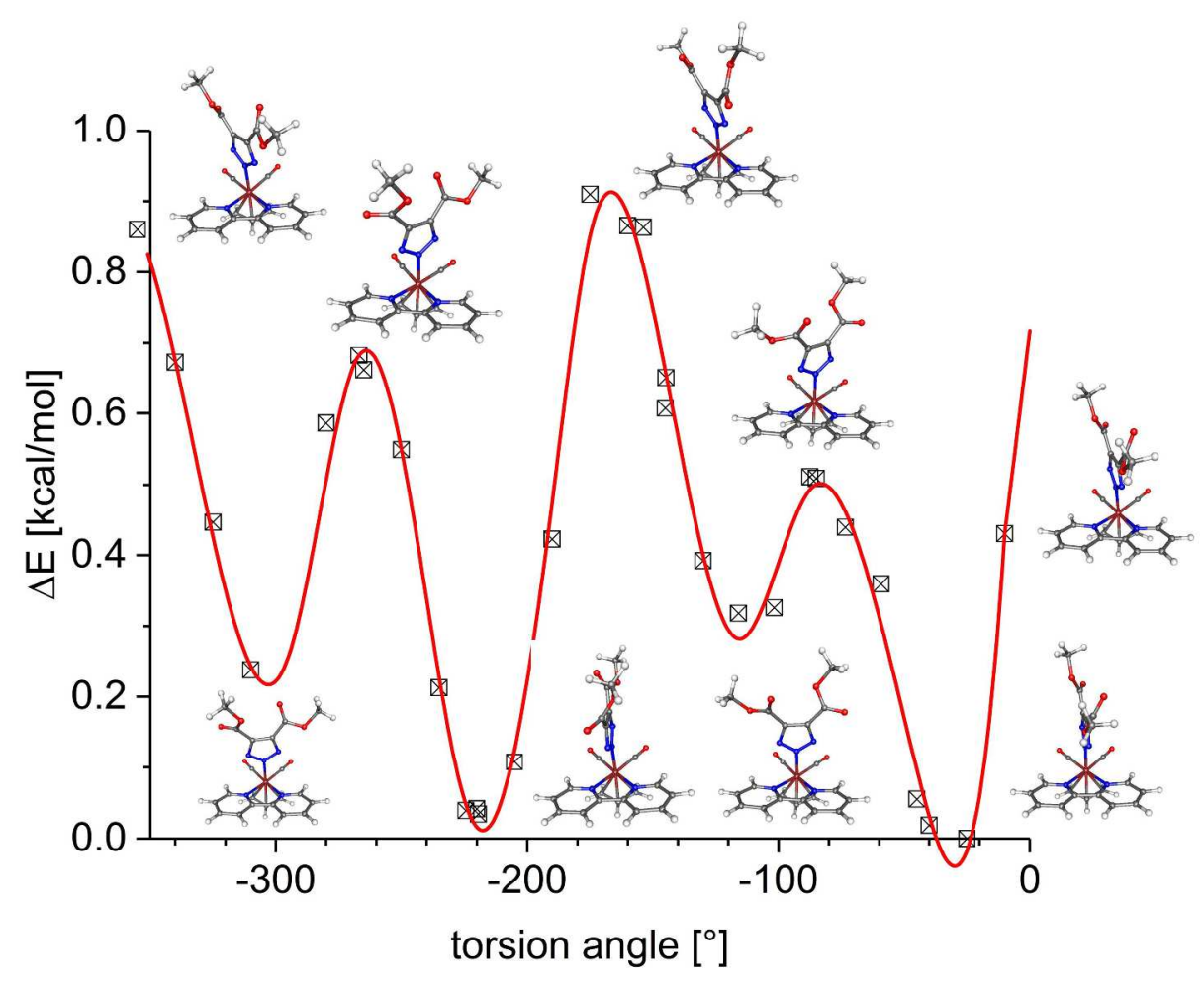

$269 \times 206 \mathrm{~mm}(300 \times 300$ DPI $)$ 


\section{Journal Name}

\section{ARTICLE}

Received 00th January 20xx, Accepted 00th January 20xx

DOI: $10.1039 / x 0 x \times 00000 x$ www.rsc.org/

\section{Catalyst-free room-temperature iClick reaction of molybdenum(II) and tungsten(II) azide complexes with electron-poor alkynes: Structural preferences and kinetic studies}

\author{
Paul Schmid, ${ }^{a}$ Matthias Maier, ${ }^{a}$ Hendrik Pfeiffer, ${ }^{a}$ Anja Belz, ${ }^{a}$ Lucas Henry, ${ }^{a}$ Alexandra Friedrich, ${ }^{\text {a }}$ \\ Fabian Schönfeld, ${ }^{a}$ Katharina Edkins ${ }^{\mathrm{a}, \mathrm{b}}$ and Ulrich Schatzschneider ${ }^{\mathrm{a}^{*}}$
}

\begin{abstract}
Two isostructural and isoelectronic group VI azide complexes of the general formula $\left[\mathrm{M}\left(\eta^{3}\right.\right.$-allyl) $\left.\left(\mathrm{N}_{3}\right)(\mathrm{bpy})(\mathrm{CO})_{2}\right]$ with $\mathrm{M}=$ Mo, $W$ and bpy $=2,2^{\prime}$-bipyridine were prepared and fully characterized, including X-ray structure analysis. Both reacted smoothly with electron-poor alkynes such as dimethyl acetylenedicarboxylate (DMAD) and 4,4,4-trifluoro-2-butynoic acid ethyl ester in a catalyst-free room-temperature iClick [3+2] cycloaddition reaction. Reaction with phenyltrifluoromethylacetylene, on the other hand, did not lead to any product formation. X-ray structures of the four triazolate complexes isolated showed the monodentate ligand to be N2-coordinated in all cases, which requires a 1,2-shift of the nitrogen from the terminal azide to the triazolate cycloaddition product. On the other hand, a ${ }^{19} \mathrm{~F}$ NMR spectroscopic study of the reaction of the fluorinated alkyne with the tungsten azide complex at $27^{\circ} \mathrm{C}$ allowed detection of the N1-coordinated intermediate. With this method, the second-order rate constant was determined as $(7.3 \pm 0.1) \times 10^{-2} \mathrm{M}^{-1} \mathrm{~s}^{-1}$, which compares favorably with that of first-generation compounds such as difluorocyclooctyne (DIFO) used in the strainpromoted azide-alkyne cycloaddition (SPAAC). In contrast, the reaction of the molybdenum analogue was too fast to be studied with NMR methods. Alternatively, solution IR studies revealed pseudo-first order rate constants of 0.4 to $6.5 \times 10^{-3}$ $\mathrm{s}^{-1}$, which increased in the order of $\mathrm{Mo}>\mathrm{W}$ and $\mathrm{F}_{3} \mathrm{C}-\mathrm{C} \equiv \mathrm{C}-\mathrm{COOEt}>\mathrm{DMAD}$.
\end{abstract}

\section{Introduction}

Since their introduction about 15 years ago, ${ }^{1}$ "click" reactions have been an indispensable tool for drug development ${ }^{2-4}$ and also attracted considerable attention in bioorthogonal labelling. ${ }^{5-8}$ Careful optimization of the reactants has led to systems with very fast coupling kinetics ( $k$ up to $10^{5} \mathrm{M}^{-1} \mathrm{~s}^{-1}$ ), ${ }^{9,10}$ which has enabled the study of cellular processes on a timescale of minutes. ${ }^{11}$ In recent years, interest has also been directed at the development of inorganic "click" (iClick) reactions which involve small ligands directly coordinated to a metal center. ${ }^{12,}{ }^{13}$ In particular, the [3+2] cycloaddition reaction of metal-azide complexes with terminal and internal alkynes as well as nitriles gives rise to metal-triazolate and metal-tetrazolate complexes, respectively. ${ }^{14-16}$ Very recently, dual-functional platinum(II) complexes incorporating both a metal-coordinated alkyne and a peripheral organoazide moiety have also been sucessfully polymerized to metallopoly-

\footnotetext{
a. Institut für Anorganische Chemie, Julius-Maximilians-Universität Würzburg, Am Hubland, D-97074 Würzburg, Germany, +49 9313183636

ulrich.schatzschneider@uni-wuerzburg.de.

${ }^{b .}$ School of Pharmacy, Queen's University Belfast, 97 Lisburn Road, Belfast BT9 7BL, United Kingdom

Electronic Supplementary Information (ESI) available: Synthesis of ligand and metal complex precursors; molecular structures of 2, 5, 6, and 11; crystallographic parameters for all X-ray structures; IR spectra of precursors and kinetic traces; DFTcalculated conformational energy diagramm of 6. See DOI: 10.1039/x0xx00000x
}

triazolates, however under addition of copper(II) acetate as a catalyst. ${ }^{17}$ While initial kinetic studies by Veige et al. were focused on linear gold(I) azide compounds, ${ }^{18}$ we have recently shown for a series of $\mathrm{Cp}^{*} \mathrm{Rh}(\mathrm{III})$ azide complexes with substituted 2,2'-bipyridine (bpy) coligands that the rate of the reaction is accelerated by introduction of electron-donating groups in the 4- and 4'-position of the bpy. ${ }^{19}$ However, the effect of variation of metal center and alkyne on the speed of the iClick reaction has not been investigated so far. Herein, we report on the synthesis and structural characterization of two isoelectronic molybdenum(II) and tungsten(II) azide complexes $\left[\mathrm{M}\left(\eta^{3}\right.\right.$-allyl) $\left.\left(\mathrm{N}_{3}\right)(\mathrm{bpy})(\mathrm{CO})_{2}\right]$ and their iClick cycloaddition reaction with two different electron-deficient alkynes, dimethyl acetylenedicarboxylate (DMAD) and 4,4,4-trifluoro-2butynoic acid ethyl ester, which are formally related to each other by substitution of a methyl ester by a trifluoromethyl group. In particular, ${ }^{19} \mathrm{~F}$ NMR studies involving the latter coupling partner were aimed at identifying the initial N1bound triazolate species which should form from the terminal azide ligand in the starting material, while essentially all iClick products with substituents in the 4- and 5-position of the triazolate ring structurally characterized so far are N2coordinated, ${ }^{20-28}$ with some rare N1-bound structures possibly enforced by increased steric bulk on the substituents. ${ }^{29}$

\section{Results and discussion}




\section{Synthesis}

The azide complexes $\left[\mathrm{M}\left(\eta^{3}\right.\right.$-allyl $\left.)\left(\mathrm{N}_{3}\right)(\mathrm{bpy})(\mathrm{CO})_{2}\right]$ with $\mathrm{M}=\mathrm{Mo}$ (2) and $\mathrm{W}$ (10) were prepared from the corresponding halides 1 and 9, respectively. In the case of the molybdenum compound, a one-pot reaction of $\left[\mathrm{Mo}(\mathrm{CO})_{6}\right]$ with allyl chloride and 2,2'-bipyridine in tetrahydrofuran afforded 1 in $76 \%$ yield. ${ }^{30}$ However, the corresponding tungsten complex was not accessible via this route due to considerably slower ligand exchange on the $5 \mathrm{~d}$ vs. $4 \mathrm{~d}$ metal center. Therefore, a consecutive reaction sequence had to be employed. Starting from $\left[\mathrm{W}(\mathrm{CO})_{6}\right]$, two carbonyl ligands were oxidized to carbon dioxide by addition of 2 eq. of trimethylamine $N$-oxide (TMAO), ${ }^{31}$ which facilitates the introduction of the 2,2'bipyridine ligand under mild conditions, leading to $\left[\mathrm{W}(\mathrm{bpy})(\mathrm{CO})_{4}\right](7)$, although only in a low efficiency of $31 \%$. An additional carbonyl ligand was then replaced by pyridine upon reflux in xylene for $18 \mathrm{~h}$, leading to $\left[\mathrm{W}(\mathrm{bpy})(\mathrm{CO})_{3}(\mathrm{py})\right](8)$ in $76 \%$ yield. $^{32}$ The mixed-ligand bipyridine/pyridine complex then underwent oxidative addition of allyl chloride to give $\left[\mathrm{W}\left(\eta^{3}\right.\right.$-allyl $\left.) \mathrm{Cl}(\mathrm{bpy})(\mathrm{CO})_{2}\right](9)$ in a yield of $86 \%$. Introduction of the azide ligands also required different procedures. In the case of molybdenum, the chlorido complex 1 was first treated with silver triflate to precipitate the halide as insoluble silver chloride, followed by reaction with a 2 -fold excess of sodium azide in acetonitrile at room temperature overnight, to give $\mathbf{2}$ in $58 \%$ yield. The analogous tungsten compound, on the other hand, was obtained by refluxing chlorido complex 9 in a mixture of acetone/methanol in the presence of 5 eq. of sodium azide for $6 \mathrm{~h}$, which allowed isolation of 10 in $62 \%$ yield.

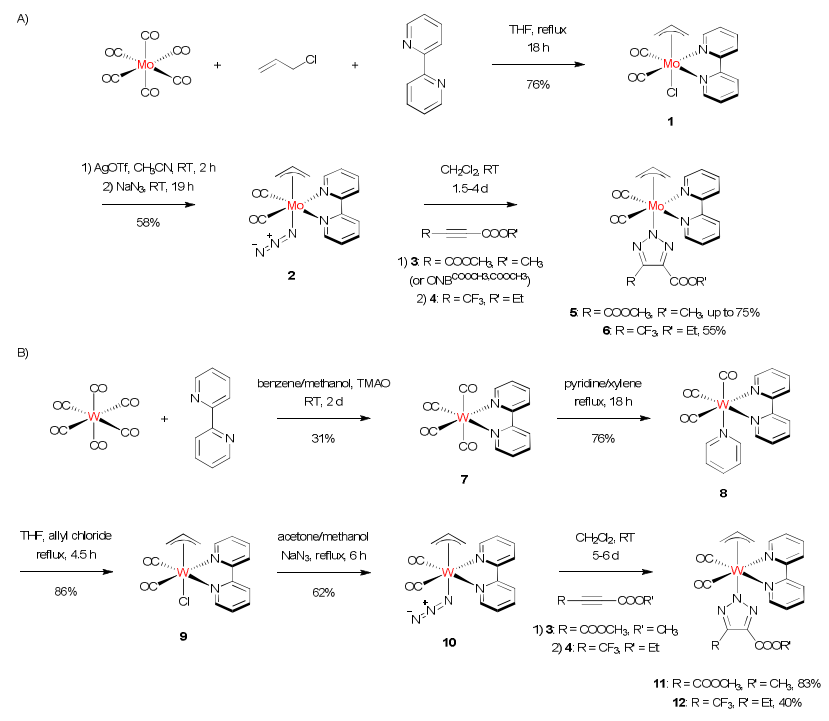

Scheme 1 Synthesis of molybdenum and tungsten triazolate complexes 5, 6, 11, and $\mathbf{1 2}$ by catalyst-free room temperature "iClick" reaction of the corresponding azide compounds with electron poor alkynes dimethyl acetylenedicarboxylate (DMAD, 3) and 4,4,4-trifluoro-2-butynoic acid ethyl ester (4) or "masked" alkyne dimethyl-7-oxa-

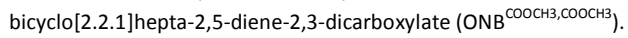

The molybdenum and tungsten azide compounds $\mathbf{2}$ and $\mathbf{1 0}$ were then tested for their efficiency in the catalyst-free "iclick" cycloaddition reaction with electron-poor alkynes dimethyl acetylenedicarboxylate (DMAD, 3) and 4,4,4-trifluoro-2-butynoic acid ethyl ester (4). Using these coupling partners, the [3+2] cycloaddition reaction proceeded smoothly at room temperature in dichloromethane, from which the triazolate complexes 5, 6, 11, and 12 were easily isolated by precipitation with diethyl ether or $n$-hexane in moderate to good yield (36-85\%). Alternatively, a "masked" alkyne in the form of dimethyl-7-oxa-bicyclo[2.2.1]hepta2,5-diene-2,3-dicarboxylate $\left(\mathrm{ONB}^{\mathrm{COOCH} 3, \mathrm{COOCH}^{3}}\right)$ was also utilized in the cycloaddition with $\mathbf{2}^{33}$ which gave an even higher yield of triazolate 5 compared to the reaction with DMAD (3). To further evaluate the scope of dipolarophiles, phenyltrifluoromethylacetylene (15) was prepared in a three-step published procedure with the pyrolysis of triphenylphosphonium- $\alpha$-(trifluoroacetyl)benzylide (14) as the key step (Scheme S1). ${ }^{34-40}$ However, no product could be isolated from a mixture of molybdenum azide complex $\mathbf{2}$ and alkyne $\mathbf{1 5}$ upon reaction in dichloromethane at room temperature for $24 \mathrm{~h}$. A change of the solvent to acetone and increase of the reaction temperature to reflux did not improve the outcome and in both cases, the precipitated red solid turned out to be the molybdenum azide starting material 2 based on ${ }^{1} \mathrm{H}$ and ${ }^{19} \mathrm{~F}$ NMR analysis. Either the presence of only one electron-withdrawing group is insufficient for a proper match of frontier orbitals or the phenyl group is sterically too demanding to allow approach to the azide reaction partner.

\section{Spectroscopy and X-ray structure analysis}

The strong and well-separated vibrational bands of the azide, carbonyl, and triazolate ester groups provide an easy handle for the identification of the reaction products with IR spectroscopy. In particular, the successful substitution of the chlorido by azide ligand is evident from the strong azide antisymmetric stretch present in $\mathbf{2}$ and $\mathbf{1 0}$ at 2036 and 2046 $\mathrm{cm}^{-1}$, respectively (Table 1 ). ${ }^{41}$ In the course of the iClick cycloaddition reaction with alkynes, $v_{\text {asym }}\left(\mathrm{N}_{3}\right)$ disappears and instead, the $\mathrm{C}=\mathrm{O}$ stretches of the ester carbonyl groups on the triazolate ring formed are observed at about 1710 to 1725 $\mathrm{cm}^{-1}$.

Table 1 Comparison of azide, carbonyl, and triazolate ester group vibrations for molybdenum and tungsten complexes $\left[\mathrm{M}\left(\eta^{3}\right.\right.$-allyl $\left.) \mathrm{X}(\mathrm{bpy})(\mathrm{CO})_{2}\right] \mathbf{1}, \mathbf{2}, \mathbf{5}$, and $\mathbf{6}$ as well as 9-12 (in $\left.\mathrm{cm}^{-1}\right)$.

\begin{tabular}{|c|c|c|c|}
\hline$x$ & molybdenum & tungsten & $\begin{array}{c}\text { difference } \\
v(\mathrm{~W})-\mathrm{v}(\mathrm{Mo})\end{array}$ \\
\hline \multirow{2}{*}{$\mathrm{Cl}$} & $1925(\mathrm{C} \equiv \mathrm{O})$ & 1915 (C三O) & -10 \\
\hline & $1832(C \equiv 0)$ & 1819 (C三O) & -13 \\
\hline \multirow{3}{*}{$\mathrm{N}_{3}$} & $2036\left(N_{3}\right)$ & $2046\left(N_{3}\right)$ & +10 \\
\hline & $1928(\mathrm{C} \equiv \mathrm{O})$ & 1920 (C三O) & -8 \\
\hline & $1836(\mathrm{C} \equiv \mathrm{O})$ & $1821(\mathrm{C} \equiv 0)$ & -15 \\
\hline \multirow{3}{*}{ triazolate ${ }^{\mathrm{COOCH} 3, \mathrm{COOCH} 3}$} & $1931(\mathrm{C} \equiv 0)$ & 1921 (C三O) & -10 \\
\hline & $1856(\mathrm{C} \equiv 0)$ & $1841(\mathrm{C} \equiv \mathrm{O})$ & -15 \\
\hline & $1723(\mathrm{C}=0)$ & $1724(\mathrm{C}=\mathrm{O})$ & +1 \\
\hline \multirow{3}{*}{ triazolate ${ }^{\mathrm{CF} 3, \mathrm{COOEt}}$} & $1938(C \equiv 0)$ & 1929 (C三O) & -9 \\
\hline & $1864,1854(\mathrm{C} \equiv 0)$ & $1850(C \equiv 0)$ & -9 \\
\hline & $1713(C=0)$ & $1723(C=0)$ & +10 \\
\hline
\end{tabular}

The symmetric and antisymmetric stretches of the cis- $\mathrm{M}(\mathrm{CO})_{2}$ moiety, on the other hand, are much less responsive to the 
exchange of the axial ligand. The replacement of chloride by azide results in changes $<5 \mathrm{~cm}^{-1}$ in both bands. Rather small shifts are also observed upon generation of the coordinated triazolate ligand in the course of the cycloaddition reaction and seem to depend on the further substitution pattern on the newly generated five-membered ring, with the antisymmetric stretch somewhat more responsive than the symmetric one. Replacement of the methyl ester group in the triazolates $\mathbf{5}$ and $\mathbf{1 1}$ by trifluoromethyl in $\mathbf{6}$ and $\mathbf{1 2}$ leads to somewhat larger shifts in the band positions but generally, these do not exceed about $20 \mathrm{~cm}^{-1}$.

The assignment of the ${ }^{1} \mathrm{H}$ NMR spectra is complicated by the presence of a mixture of exo and endo conformers due to two alternative orientations of the allyl group relative to the bpy ligand, with some of the signals overlapping. ${ }^{42-45}$ In addition, the triazolate ligand can assume two different coordination modes (N1 vs. N2) when carrying identical substituents in 4and 5-position, as in $\mathbf{5}$ and $\mathbf{1 1}$, while three different isomers are possible for the unsymmetrically substituted compounds 6 and 12. However, the trifluoromethyl group in alkyne $\mathbf{4}$ as well as triazolate complexes $\mathbf{6}$ and $\mathbf{1 2}$ is a sensitive and diagnostic marker for ${ }^{19} \mathrm{~F}$ NMR spectroscopy. In 4,4,4-trifluoro-2-butynoic acid ethyl ester (4), its resonance is observed at $-50.9 \mathrm{ppm}$ while in the resulting triazolate complexes, it is shifted upfield by about -6 to $-8 \mathrm{ppm}$ to approx. $-58 \mathrm{ppm}$. In the case of molybdenum compound 6 , three signals are observed in that range with an intensity ratio of $80: 14: 6$, while the tungsten analogue 12 shows only two species in 88:12 ratio. However, since the differences in chemical shift are very small, it is not possible to assign the different species to either exo/endo isomers or the different coordination modes of the triazolate ligand.

Both azide complexes $\mathbf{2}$ and $\mathbf{1 0}$ as well as the four triazolate compounds 5, 6, 11, and 12 were characterized by single-crystal $\mathrm{X}$-ray structure analysis with the molecular structures shown in Fig. 1 and 2 as well as Fig. S1-S4. Relevant crystallographic parameters are listed in Table S1. The tungsten center in $\mathbf{1 0}$ is in a pseudooctahedral coordination environment with the bpy ligand and the cis-W(CO) $)_{2}$ moiety in the central plane (Fig. 1). The azide group points away from the bpy with a W1-N1-N2 angle of $124.3(3)^{\circ}$ and is located on a mirror plane which intersects the two carbonyl ligands and the central bpy $\mathrm{C} 6-\mathrm{C} 6 \mathrm{a}$ axis, with the two halves of the molecule symmetry-related to each other.

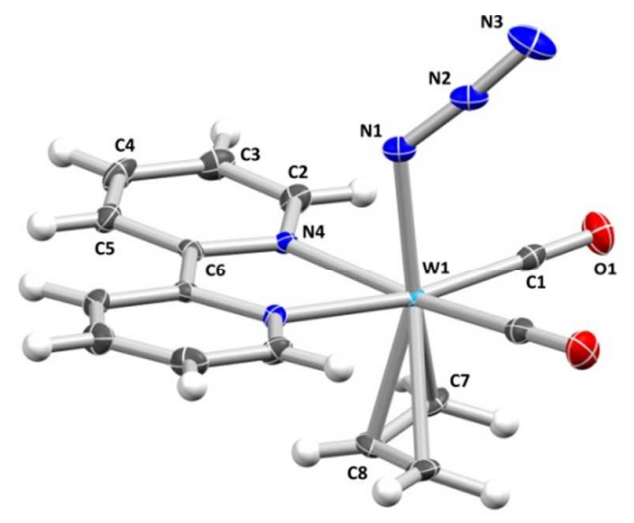

Fig. 1 Molecular structure of $\left[\mathrm{W}\left(\eta^{3}\right.\right.$-allyl $\left.)\left(\mathrm{N}_{3}\right)(\mathrm{bpy})(\mathrm{CO})_{2}\right] 10$ with thermal ellipsoids displayed at the $50 \%$ probability level. The asymmetric unit contains only one half of the molecule with the other one related to it by a mirror plane. Selected bond lengths [Å] and angles [ ${ }^{\circ}$ ] for 10: W1-C1 1.959(4), W1-N1 2.169(5), W1-N4 2.227(3), W1-(allyl centroid) 2.032(4), C1-O1 1.161(4), C7-C8 1.413(5), N1-N2 1.199(6), N2-N3 1.160(7), C1-W1-C1a 82.2(2), N4-W1-N4a 72.5(2), C1-W1-N4 102.0(2), N1-W1-(allyl centroid) 172.8(2), C7-C8-C7a 114.5(5), W1-N1-N2 124.3(3), N1-N2-N3 175.4(5).

All other parameters, in particular long N1-N2 (1.199(6) Å) and short N2-N3 (1.160(7) $\AA$ ) bond distances in the azide ligand, ${ }^{41}$ as well as C1-01 bond lengths of 1.161(4) $\AA$, are as expected. ${ }^{46}$ There are about a dozen tungsten azide complexes structurally characterized so far, ${ }^{47-55}$ but only one of them features a mixedligand carbonyl/azide coordination sphere related to $\mathbf{1 0}$. However, in $\left[\mathrm{W}\left(\eta^{3}\right.\right.$-allyl) $\left.\left(\mathrm{N}_{3}\right)(\mathrm{CO})_{2}(\mathrm{en})\right]$, the azide ligand is trans to one of the two carbonyl groups, ${ }^{53}$ while in $\mathbf{1 0}$, it is trans to the allyl moiety. The metrical parameters of molybdenum azide complex $\mathbf{2}$ are essentially identical to those of the tungsten congener 10, with differences in bond lengths and angles below $0.01 \AA$ and $0.5^{\circ}$, respectively (Fig. S1). Most closely related structurally characterized compounds include $\left[\mathrm{Mo}\left(\eta^{3} \text {-allyl) }\left(\mathrm{N}_{3}\right)(\mathrm{CO})_{2}(\mathrm{en})\right]^{53}\right.$ also isostructural to the tungsten complex mentioned above, two molybdenum dicarbonyl complexes with chelating diphosphane ligands of general formula $\left[\mathrm{Mo}\left(\eta^{3}\right.\right.$-allyl) $\left.\left(\mathrm{N}_{3}\right)(\mathrm{CO})_{2}\left(\mathrm{R}_{2} \mathrm{P}\left(\mathrm{CH}_{2}\right)_{n} \mathrm{PR}_{2}\right)\right]$, which vary in the linker length and substituent on the phosphorous center $(n=2, \mathrm{R}=$ $\mathrm{C}_{6} \mathrm{H}_{5}$ vs. $\left.n=1, \mathrm{R}=\mathrm{CH}_{3}\right),{ }^{22,56}$ and $\left[\mathrm{Mo}\left(\mathrm{N}_{3}\right)(\mathrm{NO})\left(\mathrm{bpm}^{\mathrm{CH} 3, \mathrm{CH} 3}\right)(\mathrm{CO})_{2}\right]$ with $\mathrm{bpm}^{\mathrm{CH} 3, \mathrm{CH} 3}=$ bis(3,5-dimethyl-pyrazol-1-yl)methane. ${ }^{57}$ However, in all these compounds, the azide ligand is oriented trans to one of the diatomic ligands, either $\mathrm{CO}$ or NO, not the allyl.

All four triazolate complexes 5, 6, 11, and $\mathbf{1 2}$ obtained by iClick reaction with the electron-poor alkynes dimethyl acetylenedicarboxylate (DMAD, 3) or 4,4,4-trifluoro-2-butynoic acid ethyl ester (4) exhibit N2-coordination of the five-membered ring (Fig. 2 and Fig. S2-S4), similar to the other compounds prepared this way we have characterized so far. ${ }^{19,} 28$ Since the azide ligand is N1coordinated in $\mathbf{2}$ and 10, apparently a 1,2-shift has to take place during or after the cycloaddition reaction. The bond lengths and angles of all four triazolate complexes 5, 6, 11, and 12 are essentially identical, with deviations below $0.02 \AA$ and $0.3^{\circ}$, respectively. Apparently, the influence of the metal center (Mo vs. W) and substituent in the 4- and 5-position on the triazolate ring (methyl ester vs. trifluoromethyl/ethyl ester) on these parameters is negligible.

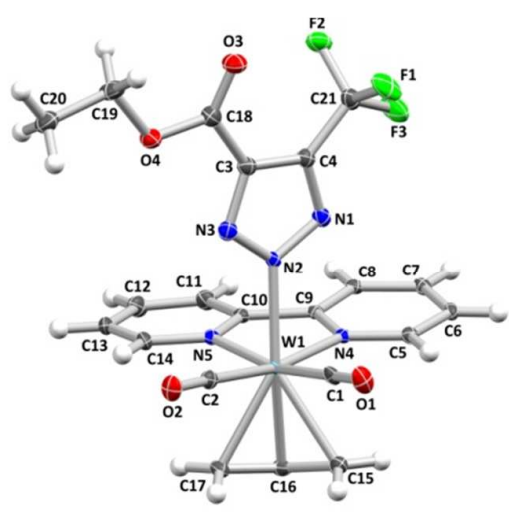


Fig. 2 Molecular structure of $\left[\mathrm{W}\left(\eta^{3}\right.\right.$-allyl)(triazolate $\left.\left.{ }^{\mathrm{CF} 3, \mathrm{COOCH} 3}\right)(\mathrm{bpy})(\mathrm{CO})_{2}\right] 12$ with thermal ellipsoids displayed at the $50 \%$ probability level. Selected bond lengths $[\AA \AA]$ and angles [ ${ }^{\circ}$ for 12: W1-C1 1.957(2), W1-C2 1.965(2), W1-N2 2.206(2), W1-N4 2.213(2), W1-N5 2.224(2), W1-(allyl centroid) 2.031(2), C1-01 1.163(3), C2-O2 1.158(2), C15-C16 1.415(4), C16-C17 1.413(4), C1-W1-C2 78.0(1), N4-W1-N5 73.02(7), C1-W1-N4 105.22(9), C2-W1-N5 101.89(9), N2-W1-(allyl centroid) 176.27(7), C15-C16-C17 115.1(2), (N2-W1-C16)-(triazolate) 49.5(3).

The same is also true when comparing the azide vs. triazolate complexes, where differences of key parameters are below $0.02 \AA$ and $0.8^{\circ}$, respectively. One notable exception, however, is the orientation of the triazolate ring plane relative to the central $\mathrm{C}-\mathrm{C}$ axis of the bpy ligand. In the trifluoromethyl-substituted molybdenum compound $\mathbf{6}$, these two are essentially parallel to each other and the triazolate assumes a staggered orientation relative to the two bipyridine $\mathrm{N}$-metal-CO angles. In contrast, in the other three complexes $\mathbf{5}, \mathbf{1 1}$, and $\mathbf{1 2}$, the triazolate ring plane is significantly rotated around the $\mathrm{M}-\mathrm{N} 2$ axis relative to the central bpy $\mathrm{C}-\mathrm{C}$ vector and assumes a more or less eclipsed orientation relative to one of the $\mathrm{M}-\mathrm{CO}$ moieties. In the absence of any notable intramolecular interactions, the twist around the metal-triazolate $\mathrm{N} 2$ bond is apparently governed by subtle packing effects in the solid state. A detailed analysis of the energy barrier between the different conformations based on DFT calculations is presented below.

\section{${ }^{19}$ F NMR kinetic measurements}

The rate constant of the cycloaddition reaction is a very important parameter when this method is to be applied to bioconjugation, since it has to be faster than the biological process of interest. With 4,4,4-trifluoro-2-butynoic acid ethyl ester (4) as the alkyne component, the use of ${ }^{19} \mathrm{~F} \mathrm{NMR}$ spectroscopy is a facile way to follow the course of the reaction, since there is a shift of about $-8 \mathrm{ppm}$ between the alkyne $\mathrm{CF}_{3}$ signal, ${ }^{28}$ which is observed at $-50.9 \mathrm{ppm}$ in DMSO$d_{6}$, and that of the trifluoromethyl group in the 4-position of the molybdenum- and tungsten-coordinated triazolates resulting from the cycloaddition, where the peaks are found at around $-58 \mathrm{ppm}$, with very little variance between the two metals.

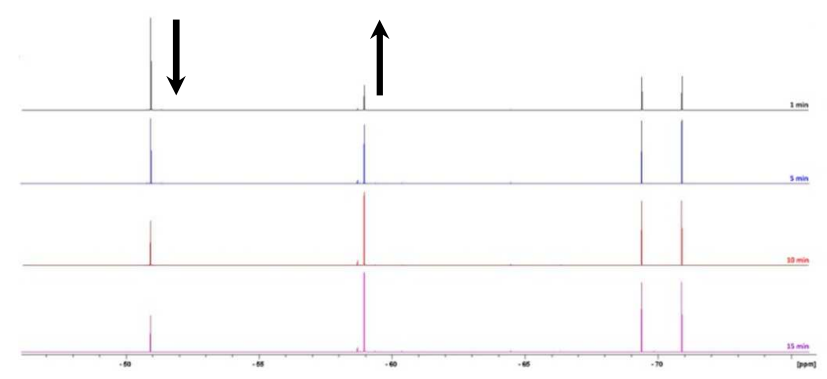

Fig. 3 Changes in the ${ }^{19} \mathrm{~F}$ NMR spectra $\left(470.59 \mathrm{MHz}\right.$, DMSO- $d_{6}$ ) of a mixture of tungsten azide complex 10 (35 mM), 4,4,4-trifluoro-2-butynoic acid ethyl ester (4, $35 \mathrm{mM}$ ) and ammonium hexafluorophoshate $(17 \mathrm{mM})$ at $27{ }^{\circ} \mathrm{C}$ over $1-15 \mathrm{~min}$. Due to an experimental lag time no data could be obtained for the initial phase of the reaction $<1$ $\min$.

Thus, the tungsten azide complex 10 was dissolved in DMSO- $d_{6}$ and mixed with ammonium hexafluorophosphate serving as an internal standard. Then, alkyne $\mathbf{4}$ was added with the metal azide complex-to-alkyne ratio adjusted to $1: 1$. A series of ${ }^{19} \mathrm{~F} \mathrm{NMR}$ spectra were recorded at $27{ }^{\circ} \mathrm{C}$ in $40-50 \mathrm{~s}$ intervals over $30 \mathrm{~min}$, with a time lag of about $1 \mathrm{~min}$ between the mixing and the first spectrum obtained due to experimental limitations. In addition to the signal of the alkyne at $-50.9 \mathrm{ppm}$ and the doublet of the hexafluorophosphate anion at -70.1 ppm with a ${ }^{1} J_{F, P}$ coupling of $711 \mathrm{~Hz}$, already the first spectrum recorded shows two additional signals at -58.7 and $-59.0 \mathrm{ppm}$ (Fig. 3 top). With increasing reaction time, these two signals assigned to the isomeric N1- and N2-coordinated triazolate complexes increased in intensity, while the alkyne $\mathrm{CF}_{3}$ peak at $-50.9 \mathrm{ppm}$ decreased continuously with similar time profiles (Fig. 4). In contrast, the intensity of the signal of the hexafluorophosphate additive remained constant during the whole time of the measurement.

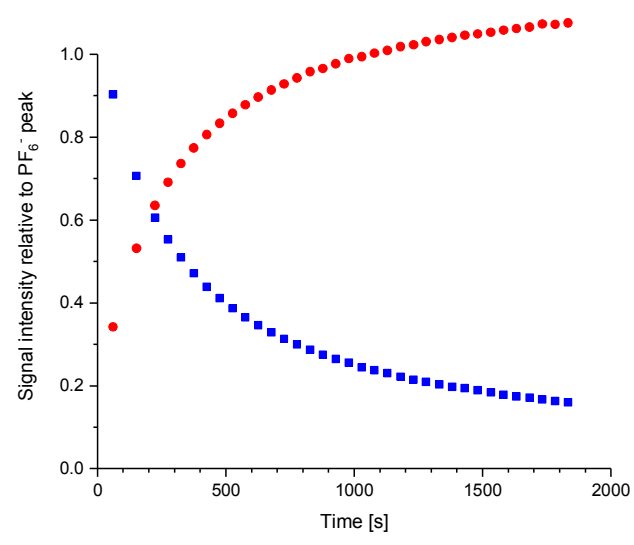

Fig. 4 Change of the ${ }^{19} \mathrm{~F}$ NMR peak intensity of the signals of the tungsten triazolate complex 12 at around -58 ppm (red circles) and of 4,4,4-trifluoro-2-butynoic acid ethyl ester (4) at $-50.9 \mathrm{ppm}$ (blue squares) with reaction time (1-30 $\mathrm{min}$ ) at $27^{\circ} \mathrm{C}$. Due to an experimental lag time no data could be obtained for the initial phase of the reaction $<1$ $\min$.

Assuming a second-order rate law and equal concentrations of azide complex and alkyne at the start of the reaction as well as negligible side-reactions, the kinetics can be modelled up to $50 \%$ conversion by eq. 1 .

$\frac{1}{\left[\text { alkyne }_{t=0}\right]}=\frac{1}{\left[\text { alkyne }_{t}\right]}+k_{2} t$

With an initial concentration of the alkyne of $35 \mathrm{mmol} \mathrm{L}^{-1}$, the second-order rate constant $k_{2}$ was determined by a linear fit from the slope of a plot of $1 /$ [alkyne eno $_{0}$ ] $-1 /$ [alkyne $_{t}$ ] over $t$ as $(7.3 \pm 0.1) \times 10^{-2} \mathrm{M}^{-1} \mathrm{~s}^{-1}$ at $27^{\circ} \mathrm{C}$. This is about one order of magnitude faster than the value of $k=7.6 \times 10^{-3} \mathrm{M}^{-1} \mathrm{~s}^{-1}$ reported by Veige and coworkers for the iClick reaction of $\left[\mathrm{Au}\left(\mathrm{N}_{3}\right)\left(\mathrm{PPh}_{3}\right)\right]$ with $\left[\mathrm{Au}\left(\mathrm{C} \equiv \mathrm{C}-\mathrm{C}_{6} \mathrm{H}_{5}\right)\left(\mathrm{PPh}_{3}\right)\right]{ }^{18}$ The rate constant determined for the iClick reaction of the tungsten azide complex is comparable to those reported, for example, for the strain-promoted azide-alkyne cycloaddition (SPAAC) of firstgeneration compounds such as difluorocyclooctyne (DIFO), but about 1-2 orders of magnitude slower than values obtained for the latest reagents such as aza-dibenzocyclooctyne (DIBAC), biarylazacyclooctynone (BARAC), and 3,3,6,6- 
tetramethyl-thiacycloheptyne (TMTH). ${ }^{9}$ Other bioorthogonal coupling reactions such as the tetrazine ligation show even faster kinetics with rate constants up to $10^{4} \mathrm{M}^{-1} \mathrm{~s}^{-1} .{ }^{10}$ The same method was also attempted to study the kinetics of the iClick reaction of the related molybdenum azide complex $\mathbf{2}$ with alkyne 4. However, the reaction proceeded very fast at room temperature and after 2-3 $\mathrm{min}$, no signal for the trifluoromethyl-substituted alkyne was detectable at -50.9 ppm. This is a strong indication that the type of metal center (Mo vs. W) has a significant influence on the rate constant, but apparently the NMR spectroscopic method successfully applied to the tungsten complex has too long a time lag between mixing and recording of the first spectrum as well as too long delay times between the individual measurements to follow the process in the case of the molybdenum compound.

\section{Solution IR kinetic measurements}

Since the iClick reaction was too fast to be monitored by NMR in the case of the molybdenum complex $\mathbf{2}$ and comparison of the cycloaddition reactions of the azide complexes with DMAD (3) was not possible using this method, since it lacks the fluorine label as in 4,4,4-trifluoro-2-butynoic acid ethyl ester (4), solution IR measurements were explored as an alternative method to obtain the rate constants for the iClick reaction of 2 and $\mathbf{1 0}$ with the two alkynes $\mathbf{3}$ and $\mathbf{4}$, as previously used successfully to study the cycloaddition reaction of $\left[\mathrm{Rh}\left(\mathrm{Cp}^{*}\right)\left(\mathrm{N}_{3}\right)\left(\mathrm{bpy}^{\mathrm{R}, \mathrm{R}}\right)\right] \mathrm{CF}_{3} \mathrm{SO}_{3}$ with alkyne $4 .^{19}$ Thus, the azide complex (2 or 10, $8 \mathrm{mM}$ ) dissolved in dimethylsulfoxide was mixed with alkyne ( 3 or $4,40 \mathrm{mM}$ ) and then quickly transferred to a liquid IR cell, with a maximum time lag of about 1.5 min between mixing and start of the measurement. Measurements in the $1800-2100 \mathrm{~cm}^{-1}$ spectral range were carried out in $20 \mathrm{~s}$ intervals until the azide band at 2050-2060 $\mathrm{cm}^{-1}$ had completely disappeared. No spectral overlap was observed in that region with bands assigned to alkynes $\mathbf{3}$ and $\mathbf{4}$ (Fig. S5). In contrast to the azide band, the two signals due to the antisymmetric and symmetric $\mathrm{C} \equiv \mathrm{O}$ stretching vibrations between 1835 and $1935 \mathrm{~cm}^{-1}$ remained essentially unchanged during the course of the reaction (Fig. 5 and Fig. S6). A minor intermediate decrease in the intensity of the symmetric $\mathrm{C} \equiv \mathrm{O}$ band might be due to subtle differences in the extinction coefficients of this vibration in the azide vs. triazolate species. Significant differences in the time required for the reaction to reach completion are clearly evident in a plot of the intensity of the azide stretch vs. reaction time (Fig. 6). The reaction of the azide complexes 2 and 10 with 4,4,4-trifluoro-2-butynoic acid ethyl ester (4) is faster than that with DMAD (3) regardless of the metal complex, while the molybdenum azide complex reacts faster than the tungsten analogue irrespective of the alkyne. The data was fit to a monoexponential decay according to eq. 2 with a correlation coefficient of better than 0.9990 for all four reactions and gave pseudo-first order rate constants $k$ in the range of 0.4 to $6.5 \times 10^{-3} \mathrm{~s}^{-1}$ (Table 2).

$A\left(\mathrm{~N}_{3}\right)=A_{1} \cdot e^{-\frac{t}{t_{1}}}+y_{0}$
The molybdenum azide complex 2 reacts about 3-4 times faster than the tungsten analogue 10, while the cycloaddition reaction with 4,4,4-trifluoro-2-butynoic acid ethyl ester (4) is 4-7 times more rapid than the one with DMAD (3).
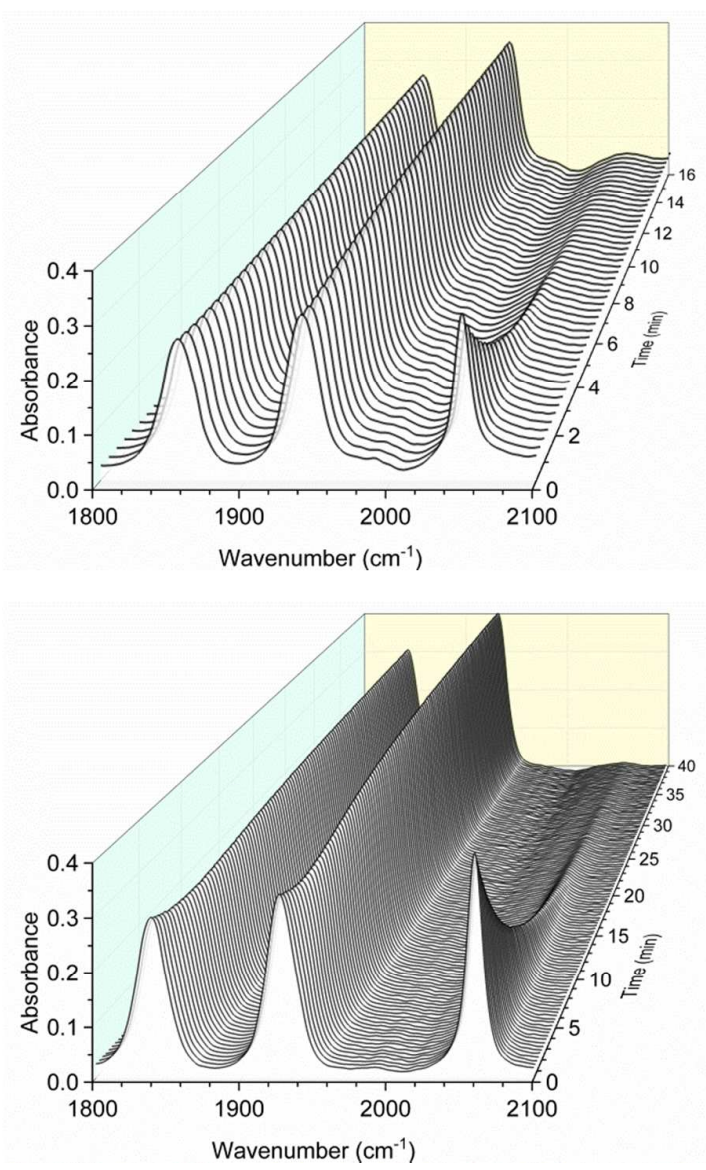

Fig. 5 Changes in the $1800-2100 \mathrm{~cm}^{-1}$ spectral range of the IR spectrum of a mixture of $\mathbf{2}$ (top) or $\mathbf{1 0}$ (bottom) in dimethylsulfoxide $(8 \mathrm{mM}$ ) upon reaction with alkyne 4 (40 $\mathrm{mM}$ ) at $26^{\circ} \mathrm{C}$ in $20 \mathrm{~s}$ intervals for up to $40 \mathrm{~min}$.

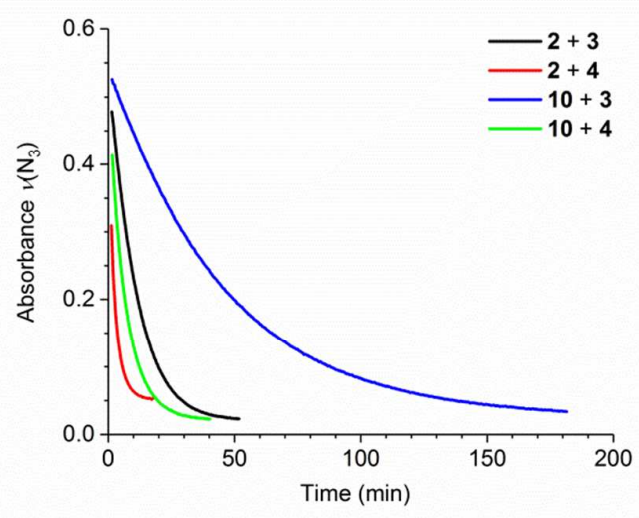

Fig. 6 Changes in the intensity of the azide stretch of complexes 2 or $10(8 \mathrm{mM})$ upon reaction with alkynes 3 or $4(40 \mathrm{mM})$ in dimethylsulfoxide at $26^{\circ} \mathrm{C}$ for up to $180 \mathrm{~min}$. 
While the latter trend corresponds well with an increase in the rate constant with increasing electron density on one of the organic reactants, as observed previously for $\left[\mathrm{Rh}\left(\mathrm{Cp}^{*}\right)\left(\mathrm{N}_{3}\right)\left(\mathrm{bpy}^{\mathrm{R}, \mathrm{R}}\right)\right] \mathrm{CF}_{3} \mathrm{SO}_{3},{ }^{19}$ and reflected in the Hammett constants for $\mathrm{COOCH}_{3}$ vs. $\mathrm{CF}_{3}$ of 0.45 and 0.54 , respectively, ${ }^{58}$ the influence of the metal on the speed of the iClick reaction has not been studied so far and thus there is no data available for comparison. Unfortunately, the isostructural chromium azide complex was not accessible for comparison due to low stability of the starting materials.

Table 2 Pseudo-first order rate constants $k$ and half-lives $t_{1 / 2}$ for the cycloaddition reaction of azide complexes $\mathbf{2}$ or $\mathbf{1 0}$ with alkynes $\mathbf{3}$ or $\mathbf{4}$ in dimethylsulfoxide at $26^{\circ} \mathrm{C}$.

\begin{tabular}{ccccc}
\hline reactants & metal & alkyne & $k\left(\right.$ in $\left.10^{-3} \mathrm{~s}^{-1}\right)$ & $t_{1 / 2}$ (in s) \\
\hline $\mathbf{1 0 + 3}$ & W & DMAD & $0.4 \pm 0.1$ & 1896 \\
$\mathbf{2}+\mathbf{3}$ & Mo & DMAD & $1.6 \pm 0.1$ & 448 \\
$\mathbf{1 0 + 4}$ & W & $\mathrm{F}_{3} \mathrm{C}-\mathrm{C} \equiv \mathrm{C}-\mathrm{COOEt}$ & $2.5 \pm 0.1$ & 274 \\
$\mathbf{2}+\mathbf{4}$ & Mo & $\mathrm{F}_{3} \mathrm{C}-\mathrm{C} \equiv \mathrm{C}$-COOEt & $6.5 \pm 0.1$ & 107 \\
\hline
\end{tabular}

Overall, however, the rate constants compare well with those of the abovementioned $\mathrm{RhCp}^{*}$ complexes $^{19}$ of 2.4 to $3.8 \times 10^{-3}$ $\mathrm{s}^{-1}$ and the ones reported by Veige et al. for the iClick reaction of $\left[\mathrm{Au}\left(\mathrm{N}_{3}\right)\left(\mathrm{PPh}_{3}\right)\right]$ with gold(I) phenylacetylides. ${ }^{59}$

\section{DFT calculations}

In order to obtain some insight into the energy hypersurface of the triazolate complexes, in particular with regard to the orientation of the allyl ligand (exo vs. endo), the coordination mode of the five-membered ring ( $N 1$ vs. N2), and the rotation of the triazolate around the metal- $N$ axis, DFT calculations were carried out on one of the molybdenum complexes serving as a model system, using the BP86 functional, a TZVP basis set, and the COSMO solvation model using dimethylsulfoxide, as also employed in the NMR studies.

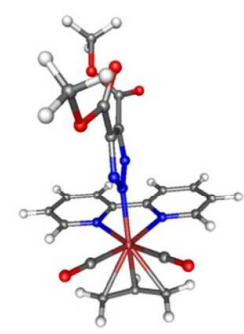

0.0

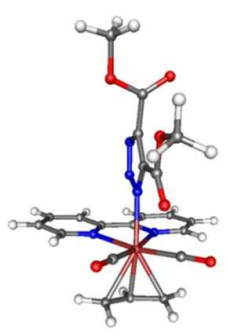

6.9

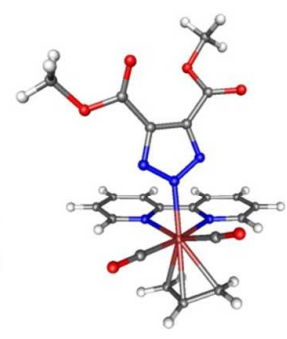

6.2
Fig. 7 DFT-optimized structures (ORCA 2.8, BP86, RI, TZVP, COSMO in DMSO) of (left) the N2-coordinated endo isomer, (center) the N1-coordinated exo isomer, and (right) the $\mathrm{N} 2$-coordinated endo isomer of $\mathbf{5}$. Relative energies are reported in $\mathrm{kcal} \mathrm{mol}^{-1}$.

A full geometry optimization without constraints showed the $\mathrm{N} 2$-coordinated endo isomer of $\mathbf{5}$ to be $6.2 \mathrm{kcal} \mathrm{mol}^{-1}$ more stable than the exo one. Retaining the endo orientation of the allyl ligand, the corresponding N1 species was destabilized by $6.9 \mathrm{kcal} \mathrm{mol}^{-1}$ relative to the reference isomer (Fig. 7), which is also confirmed by other recent structural studies. ${ }^{60,61}$ Thus, the triazolate coordination mode and allyl ligand orientation observed in the X-ray structure of $\mathbf{5}$ is indeed the most stable of the three isomers studied. However, due to the many degrees of freedom, the additional effect of rotation of the methyl ester groups around the triazolate- $\mathrm{C} 4 / \mathrm{C} 5-\mathrm{COOCH}_{3}$ axis and variation of the $\mathrm{H}_{3} \mathrm{C}-\mathrm{O}-\mathrm{C}=\mathrm{O}$ torsion angle was not further investigated.

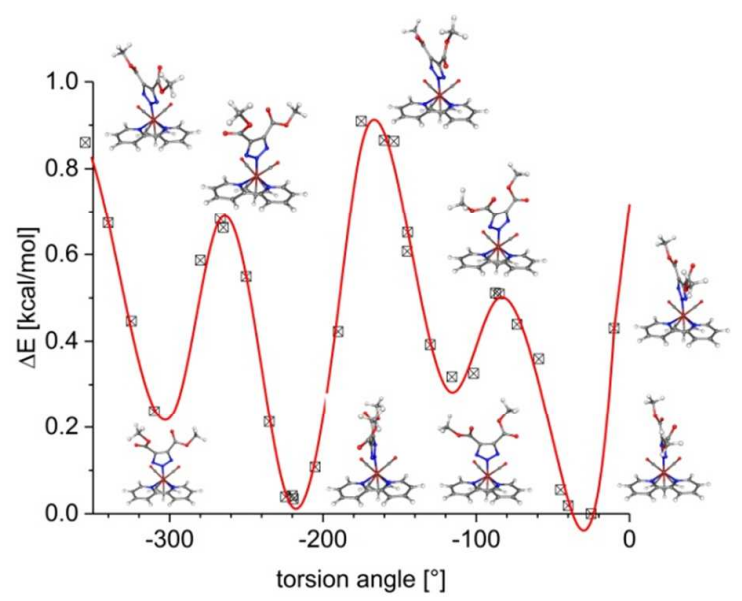

Fig. 8 Conformational energy diagram of $\mathbf{5}$ for variation of the torsion angle between the triazolate mean plane and the bpy C2-C2' axis calculated with DFT (ORCA 2.8, BP86, $\mathrm{RI}, \mathrm{TZVP}$, COSMO in DMSO).

A relaxed surface scan of the N2-coordinated endo isomer with rotation of the triazolate around the Mo-N2 bond in $15^{\circ}$ steps showed the "parallel" orientation of the triazolate mean plane and the central bpy $\mathrm{C}-\mathrm{C}$ axis as well as the "perpendicular" conformation with the triazolate at $90^{\circ}$ relative to the bipyridine central axis to be minimum structures, with the latter slightly lower in energy by about 0.2 $\mathrm{kcal} \mathrm{mol}^{-1}$, while the eclipsed orientations are transition states between the four minimum structures (Fig. 8). However, with a maximum difference in energy of only $0.9 \mathrm{kcal} \mathrm{mol}^{-1}$, the barrier is extremely low, even less than the $1.3 \mathrm{kcal} \mathrm{mol}^{-1}$ determined for a $\left[\mathrm{Mn}\right.$ (triazolate)(bpy) $\left.(\mathrm{CO})_{3}\right]$ derivative in a previous study ${ }^{28}$ and only about one-third of the energy difference between the staggered and eclipsed conformations of ethane of about $2.9 \mathrm{kcal} \mathrm{mol}^{-1}$. $^{62}$ In the case of the unsymmetrically substituted molybdenum complex 6, a different energy profile was observed (Fig. S7), since the "perpendicular" conformer with the trifluoromethyl group pointing towards the bpy ring is significantly more stable than the one turned by $180^{\circ}$, with the ethyl ester group towards the bipyridine. Furthermore, the eclipse conformations now also become inequivalent. Still, even in this case, the maximum barrier height does not exceed $1.3 \mathrm{kcal} \mathrm{mol}^{-1}$. Thus, the triazolate ligand can rotate freely around the Mo-N2 axis at ambient temperature and there is only a very minor influence of the substituents in 4- and 5-position on the barrier height.

\section{Conclusions}

Two group $\mathrm{VI}$ azide complexes of the general formula $\left[\mathrm{M}\left(\eta^{3}-\right.\right.$ allyl) $\left(\mathrm{N}_{3}\right)(\mathrm{bpy})(\mathrm{CO})_{2}$ ] with $\mathrm{M}=\mathrm{Mo}, \mathrm{W}$ were prepared and fully characterized, including $\mathrm{X}$-ray structure analysis, which revealed little differences between the two compounds. While 
the molybdenum(II) complex was accessible in two steps, the one-pot reaction of $\left[\mathrm{Mo}(\mathrm{CO})_{6}\right]$ with allyl chloride and 2,2'bipyridine followed by exchange of the chloride to the azide ligand, the tungsten(II) analogue required a different synthetic strategy due to significantly slower ligand exchange. Initially, two of the $\mathrm{CO}$ ligands in tungsten(0) hexacarbonyl were removed with TMAO and then replaced by bpy. This was followed by exchange of a third carbonyl ligand by pyridine in refluxing xylene. The resulting $\left[\mathrm{W}(\mathrm{bpy})(\mathrm{CO})_{3}(\mathrm{py})\right]$ was then employed in a further reaction sequence similar to the one employed for the molybdenum complex. The two azide complexes underwent smooth [3+2] cycloaddition iClick reactions with electron-poor alkynes such as dimethyl acetylenedicarboxylate (DMAD) and 4,4,4-trifluoro-2-butynoic acid ethyl ester, while phenyltrifluoromethylacetylene did not react. However, at present it is unclear whether this is due to electronic or steric factors. The four triazolate compounds obtained were characterized by X-ray structure analysis, which revealed the five-membered ring to be coordinated to the metal center via the $\mathrm{N} 2$ nitrogen atom in all cases. In contrast, ${ }^{19} \mathrm{~F}$ NMR spectra of the trifluoromethyl-substituted products gave hints of a mixture of the N1- and N2-coordinated species in solution, although the assignment is complicated by the additional exo/endo isomerism of the ally ligand. Still, the method allowed facile study of the reaction kinetics in the case of the tungsten azide complex, which gave a second-order rate constant of $(7.3 \pm 0.1) \times 10^{-2} \mathrm{M}^{-1} \mathrm{~s}^{-1}$, comparable to that observed for some first-generation cyclic alkynes used in the strain-promoted azide-alkyne cycloaddtion (SPAAC). The analogous molybdenum complex reacted too fast for study with NMR methods and attempts to also prepare the similar chromium compound did not succeed due to very low stability of the precursors. On the other hand, solution IR measurements allowed the determination of pseudo-first order rate constants for all four combinations of metal-azide complexes and alkynes, which were found to be in a range of 0.4 to $6.5 \times 10^{-3} \mathrm{~s}^{-1}$ and increased in the order of $\mathrm{Mo}>\mathrm{W}$ and $\mathrm{F}_{3} \mathrm{C}-\mathrm{C} \equiv \mathrm{C}-\mathrm{COOEt}>\mathrm{DMAD}$. Thus, for the first time, there has been a systematic evaluation of the effect of the metal center on the kinetics of the iclick cycloaddition reaction of metalcoordinated azides with electron-poor alkynes. In otherwise isostructural and isoelectronic compounds, the $4 \mathrm{~d}$ complex was consistently found to react faster than the $5 \mathrm{~d}$ analog. This demonstrates that the kinetics of the iClick reaction of metalcoordinated small ligands can be tuned not only by variation of electron-withdrawing vs. electron-donating groups in the periphery of the alkyne ${ }^{18}$ or the coligands, ${ }^{19}$ but also by exchange of the metal itself. Thus, it is expected that this reaction can be extended to additional transition metal azide complexes and such experiments are currently under way. In contrast, the choice of the alkyne seems to be much more limited, as demonstrated by the failure of phenyltrifluoromethylacetylene to give any cycloaddition product, even at elevated temperatures. More work will be required to dissect the electronic and steric factors which govern the reactivity of this component and establish a general building block approach to metal-triazolate complexes. ${ }^{13}$

\section{Experimental section}

\section{Materials and instruments}

Reactions were carried out in oven-dried Schlenk glassware under an atmosphere of pure argon or dinitrogen and reaction vessels were protected from light by wrapping with aluminium foil if necessary, in particular for the carbonyl complexes. Molybdenum and tungsten hexacarbonyl were supplied by Strem. All other chemicals were purchased from commercial sources and used as received. 4,4,4-Trifluoro-2-butynoic acid ethyl ester and dimethyl-7oxa-bicyclo[2.2.1] hepta-2,5-diene-2,3-dicarboxylate were prepared by published procedures. ${ }^{33,35}$

IR spectra of pure solid samples were obtained using a Nicolet 380 FT-IR spectrometer fitted with a smart iTR ATR accessory. The solution IR kinetic measurements were carried out with a JASCO FTIR 4100 instrument in an Omni-Cell 31800 liquid sample holder composed of calcium fluoride windows (width: $4 \mathrm{~mm}$ ) with a Teflon spacer $(d=200 \mu \mathrm{m})$.

NMR spectra were recorded on Bruker Avance $200\left({ }^{1} \mathrm{H}: 200.13\right.$ $\left.\mathrm{MHz},{ }^{13} \mathrm{C}: 50.32 \mathrm{MHz},{ }^{19} \mathrm{~F}: 188.12 \mathrm{MHz}\right)$, Avance $400\left({ }^{1} \mathrm{H}: 400.40\right.$ $\left.\mathrm{Mhz},{ }^{13} \mathrm{C}: 100.68 \mathrm{MHz},{ }^{19} \mathrm{~F}: 376.76 \mathrm{MHz}\right)$, and Avance $500\left({ }^{1} \mathrm{H}\right.$ : $500.13 \mathrm{MHz},{ }^{13} \mathrm{C}: 125.76 \mathrm{MHz},{ }^{19} \mathrm{~F}: 470.59 \mathrm{MHz},{ }^{31} \mathrm{P}: 202.46 \mathrm{~Hz}$ ) spectrometers. In the case of the ${ }^{1} \mathrm{H}$ and ${ }^{13} \mathrm{C}$ NMR, they were referenced relative to the residual signal of the solvent ${ }^{63}$ while they are given relative to $\mathrm{CFCl}_{3}$ in the case of the ${ }^{19} \mathrm{~F} \mathrm{NMR}$. Peak multiplicities are marked as singlet (s), doublet (d), doublet of doublet (dd), doublet of doublet of doublet (ddd), doublet of triplet $(d t)$, triplet $(t)$, and multiplet $(m)$, respectively and coupling constants $J$ are given in Hertz $(\mathrm{Hz})$. The elemental composition of the compounds was determined with an Elementar Vario MICRO cube $\mathrm{CHN}$ analyzer. Addition of $\mathrm{V}_{2} \mathrm{O}_{5}$ was usually required to obtain proper results in the case of the metal complexes.

\section{Synthesis}

[Mo( $\eta^{3}$-allyl)Cl(bpy)(CO) $)_{2}$ (1). To a degassed mixture of anhydrous tetrahydrofuran $(20 \mathrm{~mL})$ and allyl chloride $(1.6 \mathrm{~mL}$, $1.5 \mathrm{~g}, 19.7 \mathrm{mmol}$ ), molybdenum hexacarbonyl (500 mg, 1.9 mmol) and 2,2'-bipyridine (265 $\mathrm{mg}, 1.7 \mathrm{mmol}$ ) were added under a dinitrogen atmosphere and the mixture heated to reflux for $18 \mathrm{~h}$. The resulting red precipitate was filtered from the purple solution, washed with $n$-hexane $(4 \times 25 \mathrm{~mL})$ and dried under vacuum to give a bright red solid. Yield: 76\% (486 $\mathrm{mg}, 1.3 \mathrm{mmol}$ ). Elemental analysis (\%): calc. $\mathrm{C}_{15} \mathrm{H}_{13} \mathrm{CIMoN}_{2} \mathrm{O}_{2}$ : C 46.84, H 3.41, N 7.28, found: C 46.55, H 3.48, N 7.15; IR (ATR, $\mathrm{cm}^{-1}$ ): $3064(\mathrm{w}), 2985(\mathrm{w}), 1925$ (s, C=O), 1832 (s, C=O), 1600 $(\mathrm{m}), 1469(\mathrm{~m}), 1442(\mathrm{~m}), 1310(\mathrm{w}), 1152(\mathrm{w}), 1024(\mathrm{w}), 769$ $(\mathrm{m}) ;{ }^{1} \mathrm{H}$ NMR (DMSO- $\left.d_{6}, 200.13 \mathrm{MHz}, \mathrm{ppm}\right): \delta$ major isomer: $8.77\left(\mathrm{~d}, 2 \mathrm{H},{ }^{3} \mathrm{~J}_{\mathrm{H} 6, \mathrm{H} 5}=4.9 \mathrm{~Hz}\right.$, bpy-H6) $8.56\left(\mathrm{~d}, 2 \mathrm{H},{ }^{3} \mathrm{~J}_{\mathrm{H} 3, \mathrm{H} 4}=8.0\right.$ $\mathrm{Hz}$, bpy-H3), $8.17\left(\mathrm{dt}, 2 \mathrm{H},{ }^{3} J_{\mathrm{H} 4, \mathrm{H} 3 / \mathrm{H} 5}=8.0 \mathrm{~Hz},{ }^{4} J_{\mathrm{H} 4, \mathrm{H} 6}=1.0 \mathrm{~Hz}\right.$, bpy-H4), $7.63\left(\mathrm{t}, 2 \mathrm{H},{ }^{3} \mathrm{~J}_{\mathrm{H} 5-\mathrm{H} 4 / \mathrm{H} 6}=6.3 \mathrm{~Hz}\right.$, bpy-H5), 3.12-3.27 (m, $1 \mathrm{H}$, allyl- $\left.\mathrm{H}_{\text {meso }}\right), 3.07\left(\mathrm{~d}, 2 \mathrm{H},{ }^{3} J_{\mathrm{Hsyn}, \mathrm{Hmeso}}=6.2 \mathrm{~Hz}\right.$, allyl- $\left.\mathrm{H}_{\text {syn }}\right), 1.23$ $\left(\mathrm{d}, 2 \mathrm{H},{ }^{3} J_{\text {Hanti,Hmeso }}=8.7 \mathrm{~Hz}\right.$, allyl- $\left.\mathrm{H}_{\text {anti }}\right)$; minor isomer: $9.06(\mathrm{~d}$, $2 \mathrm{H},{ }^{3} \mathrm{~J}_{\mathrm{H} 6, \mathrm{H} 5}=3.8 \mathrm{~Hz}$, bpy-H6), $8.65\left(\mathrm{~d}, 2 \mathrm{H},{ }^{3} \mathrm{~J}_{\mathrm{H} 3, \mathrm{H} 4}=8.3 \mathrm{~Hz}\right.$, bpy$\mathrm{H} 3), 8.26\left(\mathrm{~d}, 2 \mathrm{H},{ }^{3} J_{\mathrm{H} 4, \mathrm{H} 3}=8.3 \mathrm{~Hz}, \mathrm{bpy}-\mathrm{H} 4\right), 7.79\left(\mathrm{t}, 2 \mathrm{H},{ }^{3} \mathrm{~J}_{\mathrm{H} 5}{ }^{-} \mathrm{H} 4 / \mathrm{H} 6\right.$ $=6.3 \mathrm{~Hz}$, bpy-H5), 3.79-3.91 (m, $1 \mathrm{H}$, allyl- $\left.\mathrm{H}_{\text {meso }}\right), 3.51(\mathrm{~d}, 2 \mathrm{H}$, ${ }^{3} J_{\text {Hsyn }, \text { Hmeso }}=6.2 \mathrm{~Hz}$, allyl- $\left.\mathrm{H}_{\text {syn }}\right), 1.36\left(\mathrm{~d}, 2 \mathrm{H},{ }^{3} J_{\text {Hanti,Hmeso }}=9.4 \mathrm{~Hz}\right.$, 
allyl- $\mathrm{H}_{\text {anti }}$ ); ${ }^{13} \mathrm{C}$ NMR (DMSO- $d_{6}, 50.32 \mathrm{MHz}, \mathrm{ppm}$ ): $\delta$ major isomer: 227.13 ( $\mathrm{C} \equiv \mathrm{O}), 153.30$ (bpy-C2), 151.85 (bpy-C6), 139.18 (bpy-C4), 126.27 (bpy-C5), 122.98 (bpy-C3), 70.76 (CHallyl), $54.00\left(\mathrm{CH}_{2}\right.$-allyl); minor isomer: the signal intensity was below the detection limit.

[Mo $\left(\eta^{3}\right.$-allyl)( $\left.\mathbf{N}_{3}\right)\left(\right.$ bpy) $\left.(\mathbf{C O})_{2}\right]$ (2). To a suspension of $\left[\mathrm{Mo}\left(\eta^{3}-\right.\right.$ allyl)Cl(bpy) $\left.(\mathrm{CO})_{2}\right] \quad(1,450 \mathrm{mg}, 1.2 \mathrm{mmol})$ in degassed acetonitrile $(50 \mathrm{~mL})$ was added silver triflate $(301 \mathrm{mg}, 1.2$ $\mathrm{mmol})$. A white precipitate appeared and the resulting suspension was stirred for $2 \mathrm{~h}$ under a dinitrogen atmosphere under exclusion of light. The clear red solution was then transferred with a teflon cannula into another flask containing sodium azide (152 mg, $2.3 \mathrm{mmol}$ ) and stirred at room temperature for $19 \mathrm{~h}$ while protected from light. The resulting red precipitate was filtered off, first washed with plenty of water to remove excess sodium azide, and then with ethanol $(2 \times 5 \mathrm{~mL})$ followed by diethylether $(2 \times 10 \mathrm{~mL})$ and subsequently dried under vacuum to give the product as a bright red solid. Yield: $58 \%$ (258 mg, $0.7 \mathrm{mmol}$ ). Elemental analysis (\%): calc. $\mathrm{C}_{15} \mathrm{H}_{13} \mathrm{MoN}_{5} \mathrm{O}_{2}$ : C 46.05, H 3.35, N 17.90, found: C 44.99, H 3.38, N 18.04; IR (ATR, cm ${ }^{-1}$ ): 3083 (w), 2036 (s, azide), 1928 (s, C=O), $1836(\mathrm{~s}, \mathrm{C} \equiv 0), 1600(\mathrm{~m}), 1469(\mathrm{~m}), 1440(\mathrm{~m}), 1311$ (w), $1172(w), 757(\mathrm{~m}), 733(\mathrm{w}) ;{ }^{1} \mathrm{H}$ NMR (DMSO-d, 200.13 $\mathrm{MHz}, \mathrm{ppm}): \delta$ major isomer: $8.80\left(\mathrm{dd}, 2 \mathrm{H},{ }^{3} J_{\mathrm{H} 6, \mathrm{H} 5}=5.3 \mathrm{~Hz},{ }^{4} J_{\mathrm{H} 6, \mathrm{H} 4}\right.$ $=0.9 \mathrm{~Hz}$, bpy-H6), $8.57\left(\mathrm{~d}, 2 \mathrm{H},{ }^{3} \mathrm{~J}_{\mathrm{H} 3, \mathrm{H} 4}=8.2 \mathrm{~Hz}\right.$, bpy-H3), 8.20 (dt, $\left.2 \mathrm{H},{ }^{3} J_{\mathrm{H} 4, \mathrm{H} 3 / \mathrm{H} 5}=7.9 \mathrm{~Hz},{ }^{4} J_{\mathrm{H} 4, \mathrm{H} 6}=1.2 \mathrm{~Hz}, \mathrm{bpy}-\mathrm{H} 4\right), 7.66$ (ddd, $2 \mathrm{H},{ }^{3} J_{\mathrm{H} 5, \mathrm{H} 4}=7.9 \mathrm{~Hz},{ }^{3} J_{\mathrm{H} 5, \mathrm{H} 6}=5.4 \mathrm{~Hz},{ }^{4} J_{\mathrm{H} 5, \mathrm{H} 3}=1.1 \mathrm{~Hz}$, bpy-H5), 3.18-3.23 $\left(\mathrm{m}, 1 \mathrm{H}\right.$, allyl- $\left.\mathrm{H}_{\text {meso }}\right), 3.14\left(\mathrm{~d}, 2 \mathrm{H},{ }^{3} \mathrm{~J}_{\text {Hsyn, Hmeso }}=6.7 \mathrm{~Hz}\right.$, allyl- $\left.\mathrm{H}_{\text {syn }}\right), 1.29\left(\mathrm{~d}, 2 \mathrm{H},{ }^{3} \mathrm{~J}_{\text {Hanti,Hmeso }}=8.9 \mathrm{~Hz}\right.$, allyl- $\left.\mathrm{H}_{\text {anti }}\right)$; minor isomer: $9.05(\mathrm{~m}, 2 \mathrm{H}$, bpy- $\mathrm{H} 6), 8.69(\mathrm{~m}, 2 \mathrm{H}$, bpy- $\mathrm{H} 3), 8.26(\mathrm{~m}$, $2 \mathrm{H}$, bpy-H4), $7.83(\mathrm{~m}, 2 \mathrm{H}$, bpy-H5), 3.71-3.89 (m, $1 \mathrm{H}$, allyl$\left.\mathrm{H}_{\text {meso }}\right), 3.20\left(\mathrm{~d}, 2 \mathrm{H},{ }^{3} \mathrm{~J}_{\text {Hsyn, Hmeso }}=8.1 \mathrm{~Hz}\right.$, allyl- $\left.\mathrm{H}_{\text {syn }}\right), 1.36(\mathrm{~d}, 2 \mathrm{H}$, ${ }^{3} J_{\text {Hanti,Hmeso }}=9.0 \mathrm{~Hz}$, allyl- $\left.\mathrm{H}_{\text {anti }}\right) ;{ }^{13} \mathrm{C}$ NMR (DMSO- $d_{6}, 50.32 \mathrm{MHz}$, ppm): $\delta$ major isomer: 227.20 (C三O), 153.27 (bpy-C2), 151.85 (bpy-C6), 139.48 (bpy-C4), 126.56 (bpy-C5), 123.04 (bpy-C3), 72.54 ( $\mathrm{CH}$-allyl), $55.78\left(\mathrm{CH}_{2}\right.$-allyl); minor isomer: the signal intensity was below the detection limit.

[Mo( $\eta^{3}$-allyl)(triazolate $\left.\left.{ }^{\mathrm{COOCH} 3, \mathrm{COOCH}_{3}}\right)(\mathrm{bpy})(\mathrm{CO})_{2}\right]$ (5). Method A: $\left[\mathrm{Mo}\left(\eta^{3}\right.\right.$-allyl) $\left.\left(\mathrm{N}_{3}\right)(\mathrm{bpy})(\mathrm{CO})_{2}\right] \quad(2,44 \mathrm{mg}, 0.11 \mathrm{mmol})$ and dimethyl acetylenedicarboxylate (DMAD, $17 \mu \mathrm{L}, 20 \mathrm{mg}, 0.14$ $\mathrm{mmol}$ ) were dissolved in dichloromethane $(15 \mathrm{~mL})$ and stirred for $36 \mathrm{~h}$ at room temperature under exclusion of light. The resulting red solution was filtered through Celite. After concentration under vacuum, it was layered with $n$-hexane for crystallization. After $3 \mathrm{~d}$ in the dark, the red needles obtained were filtered off, washed with $n$-hexane and dried under vacuum. Yield: $45 \%$ (28 mg, $0.05 \mathrm{mmol})$. Method B: [Mo $\left(\eta^{3}\right.$ allyl) $\left(\mathrm{N}_{3}\right)(\mathrm{bpy})(\mathrm{CO})_{2}$ ] $(62 \mathrm{mg}, 0.16 \mathrm{mmol}$ ) and dimethyl-7-oxabicyclo[2.2.1]hepta-2,5-diene-2,3-dicarboxylate $(50 \mathrm{mg}, 0.24$ $\mathrm{mmol})$ were dissolved in dichloromethane $(20 \mathrm{~mL})$ and stirred for $48 \mathrm{~h}$ at room temperature under exclusion of light. The solvent was removed under vacuum and the red residue washed with ethyl acetate $(3 \times 5 \mathrm{~mL})$ followed by $n$-hexane $(3 \times 5$ $\mathrm{mL}$ ) and subsequently dried under vacuum to give a red powder. Yield: $75 \%$ ( $65 \mathrm{mg}, 0.12 \mathrm{mmol}$ ). Experimental data is reported for the material obtained following procedure $\mathrm{B}$ : Elemental analysis (\%): calc. $\mathrm{C}_{21} \mathrm{H}_{19} \mathrm{MoN}_{5} \mathrm{O}_{6}$ : C 47.29, $\mathrm{H} 3.59, \mathrm{~N}$
13.13, found: C 47.03, H 3.58, N 13.14; IR (ATR, $\mathrm{cm}^{-1}$ ): 3071 (w), $2957(w), 1931$ (s, C=O), 1856 (s, C=O), 1723 (s, C=O), $1600(\mathrm{w}), 1439(\mathrm{~m}), 1292(\mathrm{w}), 1220(\mathrm{~m}), 1163(\mathrm{~m}), 1090(\mathrm{~m})$, $764(\mathrm{~m}) ;{ }^{1} \mathrm{H}$ NMR (DMSO- $d_{6}, 200.13 \mathrm{MHz}, \mathrm{ppm}$ ): $\delta$ major isomer: $8.97\left(\mathrm{dd}, 2 \mathrm{H},{ }^{3} J_{\mathrm{H} 6, \mathrm{H} 5}=5.5 \mathrm{~Hz},{ }^{4} J_{\mathrm{H} 6, \mathrm{H} 4}=1.6 \mathrm{~Hz}\right.$, bpy-H6), $8.54\left(\mathrm{~d}, 2 \mathrm{H},{ }^{3} \mathrm{~J}_{\mathrm{H} 3, \mathrm{H} 4}=8.0 \mathrm{~Hz}\right.$, bpy-H3), $8.16\left(\mathrm{dt}, 2 \mathrm{H},{ }^{3} \mathrm{~J}_{\mathrm{H} 4, \mathrm{H} 3 / \mathrm{H} 5}=\right.$ $8.0 \mathrm{~Hz},{ }^{4} J_{\mathrm{H} 4, \mathrm{H} 6}=1.6 \mathrm{~Hz}$, bpy-H4), 7.58 (ddd, $2 \mathrm{H},{ }^{3} J_{\mathrm{H} 5, \mathrm{H} 4}=8.0 \mathrm{~Hz}$, $\left.{ }^{3} J_{\mathrm{H} 5, \mathrm{H} 6}=5.5 \mathrm{~Hz},{ }^{4} J_{\mathrm{H} 5, \mathrm{H} 3}=1.6 \mathrm{~Hz}, \mathrm{bpy}-\mathrm{H} 5\right), 3.63\left(\mathrm{~s}, 6 \mathrm{H}, \mathrm{COOCH}_{3}\right)$, 3.31-3.33 $\left(\mathrm{m}, 2 \mathrm{H}\right.$, allyl- $\mathrm{H}_{\text {syn, }}$ overlapping with water signal), $3.06-3.21\left(\mathrm{~m}, 1 \mathrm{H}\right.$, allyl- $\left.\mathrm{H}_{\text {meso }}\right), 1.49\left(\mathrm{~d}, 2 \mathrm{H},{ }^{3}{ }^{\text {Hanti,Hmeso }}=9.0 \mathrm{~Hz}\right.$, allyl- $\left.\mathrm{H}_{\text {anti }}\right)$; minor isomer: $9.19(\mathrm{~s}, 2 \mathrm{H}$, bpy-H6), 8.52-8.57 (m, $2 \mathrm{H}$, bpy-H3), 8.18-8.25 (m, 2H, bpy-H4), 7.77-7.84 (m, $2 \mathrm{H}$, bpy-H5), 4.05-4.14 (m, $1 \mathrm{H}$, allyl- $\left.\mathrm{H}_{\text {meso }}\right), 3.62\left(\mathrm{~s}, 6 \mathrm{H}, \mathrm{COOCH}_{3}\right)$, $3.45\left(\mathrm{~d}, 2 \mathrm{H},{ }^{3} J_{\text {Hsyn, Hanti }}=6.3 \mathrm{~Hz}\right.$, allyl- $\left.\mathrm{H}_{\text {syn }}\right), 1.47-1.54(\mathrm{~m}, 2 \mathrm{H}$, allyl- $\mathrm{H}_{\text {anti }}$ ); ${ }^{13} \mathrm{CNMR}$ (DMSO- $d_{6}, 50.32 \mathrm{MHz}, \mathrm{ppm}$ ): $\delta$ major isomer: 226.31 ( $C=O), 162.38$ ( $\mathrm{C}=\mathrm{O}), 154.49$ (bpy-C2), 152.24 (bpy-C6), 139.55 (bpy-C4), 138.34 (triazolate-C4/C5), 126.36 (bpy-C5), 122.76 (bpy-C3), 81.58 ( $\mathrm{CH}$-allyl), $57.56\left(\mathrm{CH}_{2}\right.$-allyl), $51.64\left(\mathrm{COOCH}_{3}\right)$; minor isomer: the signal intensity was below the detection limit.

[Mo( $\eta^{3}$-allyl)(triazolate $\left.\left.{ }^{\mathrm{CF} 3, \mathrm{COOEt}}\right)(\mathrm{bpy})(\mathrm{CO})_{2}\right]$

(6).

$\left[\mathrm{Mo}\left(\eta^{3}\right.\right.$-allyl $\left.)\left(\mathrm{N}_{3}\right)(\mathrm{bpy})(\mathrm{CO})_{2}\right](2,44 \mathrm{mg}, 0.11 \mathrm{mmol})$ and 4,4,4trifluoro-2-butynoic acid ethyl ester $(23 \mathrm{mg}, 0.14 \mathrm{mmol})$ were dissolved in dichloromethane $(15 \mathrm{~mL})$ and stirred at room temperature under exclusion of light for $4 \mathrm{~d}$. Then, the solvent was removed under vacuum and the red residue washed with $n$-hexane $(3 \times 5 \mathrm{~mL})$. After drying under vacuum, the product was obtained as a red solid. Yield: $55 \%(35 \mathrm{mg}, 0.06 \mathrm{mmol}$ ). Elemental analysis (\%): calc. $\mathrm{C}_{21} \mathrm{H}_{18} \mathrm{~F}_{3} \mathrm{MoN}_{5} \mathrm{O}_{4}$ : $\mathrm{C} 45.26, \mathrm{H} 3.26$, $\mathrm{N}$ 12.57, found: C 45.26, H 3.34, N 12.38; IR (ATR, $\mathrm{cm}^{-1}$ ): 1938 (s, C=O), 1864 (s, C=O), 1854 (s, C=O), 1713 (m, C=O), 1604 $(\mathrm{m}), 1472(\mathrm{w}), 1441(\mathrm{~m}), 1310(\mathrm{~m}), 1161(\mathrm{~m}), 1132(\mathrm{~m}), 1049$ (m), $763(\mathrm{w}), 734(\mathrm{~m}) ;{ }^{1} \mathrm{H}$ NMR (DMSO- $\left.d_{6}, 199.93 \mathrm{MHz}, \mathrm{ppm}\right): \delta$ major isomer: $8.79\left(\mathrm{dd}, 2 \mathrm{H},{ }^{3} J_{\mathrm{H} 6, \mathrm{H} 5}=5.3 \mathrm{~Hz},{ }^{4} J_{\mathrm{H} 6, \mathrm{H} 4}=0.9 \mathrm{~Hz}\right.$, bpy-H6), $8.56\left(\mathrm{~d}, 2 \mathrm{H},{ }^{3} \mathrm{~J}_{\mathrm{H} 3, \mathrm{H} 4}=8.2 \mathrm{~Hz}, \mathrm{bpy}-\mathrm{H} 3\right), 8.18(\mathrm{dt}, 2 \mathrm{H}$, ${ }^{3} J_{\mathrm{H} 4, \mathrm{H} 3 / \mathrm{H} 5}=7.9 \mathrm{~Hz},{ }^{4} J_{\mathrm{H} 4, \mathrm{H} 6}=1.5 \mathrm{~Hz}$, bpy-H4), 7.58 (ddd, $2 \mathrm{H},{ }^{3} J_{\mathrm{H} 5, \mathrm{H} 4}$ $\left.=7.6 \mathrm{~Hz},{ }^{3} J_{\mathrm{H} 5, \mathrm{H} 6}=5.5 \mathrm{~Hz},{ }^{4} J_{\mathrm{H} 5, \mathrm{H} 3}=1.1 \mathrm{~Hz}, \mathrm{bpy}-\mathrm{H} 5\right), 4.14\left(\mathrm{q}, 2 \mathrm{H},{ }^{3} \mathrm{~J}\right.$ $\left.=7.2 \mathrm{~Hz}, \mathrm{COOCH}_{2} \mathrm{CH}_{3}\right), 1.52\left(\mathrm{~d}, 2 \mathrm{H},{ }^{3} J_{\text {Hanti,Hmeso }}=9.0 \mathrm{~Hz}\right.$, allyl$\left.\mathrm{H}_{\text {anti }}\right), 1.18\left(\mathrm{t}, 3 \mathrm{H},{ }^{3} \mathrm{~J}=7.1 \mathrm{~Hz}, \mathrm{COOCH}_{2} \mathrm{CH}_{3}\right)$, allyl- $\mathrm{H}_{\text {meso }}$ and allyl$\mathrm{H}_{\text {syn }}$, overlapping with solvent water signal; minor isomer not observed due to low signal intensity; ${ }^{13} \mathrm{C}$ NMR (DMSO- $d_{6}, 50.27$ $\mathrm{MHz}, \mathrm{ppm}$ ): $\delta$ major isomer: 154.71 (bpy-C2), 152.18 (bpy-C6), 139.62 (bpy-C4), 126.33 (bpy-C5), 122.72 (bpy-C3), 60.23 $\left(\mathrm{COOCH}_{2} \mathrm{CH}_{3}\right), 57.61\left(\mathrm{CH}_{2}\right.$-allyl), $13.88\left(\mathrm{COOCH}_{2} \mathrm{CH}_{3}\right), \mathrm{CO}$ and $\mathrm{CH}$-allyl signals not observed due to low signal intensity; minor isomer: the signal intensity was below the detection limit; ${ }^{19} \mathrm{~F}$ NMR (DMSO- $\left.d_{6}, 188.12 \mathrm{MHz}, \mathrm{ppm}\right): \delta-58.76\left(\mathrm{CF}_{3}\right.$, major species, $80 \%),-58.56\left(\mathrm{CF}_{3}\right.$, minor species, $\left.14 \%\right),-57.53\left(\mathrm{CF}_{3}\right.$, minor species, 6\%).

[W( $\eta^{3}$-allyl)Cl(bpy)(CO) $)_{2}$ (9). To a degassed mixture of anhydrous tetrahydrofuran $(35 \mathrm{~mL})$ and allyl chloride $(4.98 \mathrm{~mL}$, $4.68 \mathrm{~g}, 61.2 \mathrm{mmol})$, solid $\left[\mathrm{W}(\mathrm{bpy})(\mathrm{CO})_{3}(\mathrm{py})\right](8,1.03 \mathrm{~g}, 2.05$ $\mathrm{mmol}$ ) was added and the reaction mixture heated to reflux for $4.5 \mathrm{~h}$. The resulting dark brown precipitate was filtered from the dark violet solution, washed with $n$-hexane $(50 \mathrm{~mL})$, and dried under vacuum. Yield: $86 \%$ ( $836 \mathrm{mg}, 1.77 \mathrm{mmol}$ ). Elemental analysis (\%): calc. $\mathrm{C}_{15} \mathrm{H}_{13} \mathrm{CIN}_{2} \mathrm{O}_{2} \mathrm{~W} \cdot \mathrm{H}_{2} \mathrm{O}: \mathrm{C}$ 38.12, $\mathrm{H}$ 
2.77, N 5.93, found: C 36.02, H 3.09, N 5.84; IR (ATR, $\mathrm{cm}^{-1}$ ): $2976(\mathrm{~m}), 1915(\mathrm{~s}), 1819(\mathrm{~s}), 1601(\mathrm{~m}), 1466(\mathrm{~m}), 1445(\mathrm{~m}), 773$ (s); ${ }^{1} \mathrm{H}$ NMR (DMSO- $d_{6}, 500.13 \mathrm{MHz}, \mathrm{ppm}$ ): $\delta$ major isomer: 8.81 (ddd, $2 \mathrm{H},{ }^{3} J_{\mathrm{H} 6, \mathrm{H} 5}=5.5 \mathrm{~Hz},{ }^{4} J_{\mathrm{H} 6, \mathrm{H} 4}=1.5 \mathrm{~Hz},{ }^{5} J_{\mathrm{H} 6, \mathrm{H} 3}=0.6 \mathrm{~Hz}$ bpy-H6), $8.64\left(\mathrm{~d}, 2 \mathrm{H},{ }^{3} \mathrm{~J}_{\mathrm{H} 3, \mathrm{H} 4}=8.2 \mathrm{~Hz}, \mathrm{bpy}-\mathrm{H} 3\right), 8.24(\mathrm{dt}, 2 \mathrm{H}$, ${ }^{3} J_{\mathrm{H} 4, \mathrm{H} 3 / \mathrm{H} 5}=7.9 \mathrm{~Hz},{ }^{4} J_{\mathrm{H} 4, \mathrm{H} 6}=1.6 \mathrm{~Hz}$, bpy-H4), 7.67 (ddd, $2 \mathrm{H},{ }^{3} J_{\mathrm{H} 5, \mathrm{H} 4}$ $\left.=7.6 \mathrm{~Hz},{ }^{3} J_{\mathrm{H} 5, \mathrm{H} 6}=5.5 \mathrm{~Hz},{ }^{4} J_{\mathrm{H} 5, \mathrm{H} 3}=1.2 \mathrm{~Hz}, \mathrm{bpy}-\mathrm{H} 5\right), 2.85(\mathrm{~d}, 2 \mathrm{H}$, ${ }^{3} J_{\text {Hsyn,Hmeso }}=6.0 \mathrm{~Hz}$, allyl- $\left.\mathrm{H}_{\text {syn }}\right), 2.29-2.37\left(\mathrm{~m}, 1 \mathrm{H}\right.$, allyl- $\left.\mathrm{H}_{\text {meso }}\right)$, $1.45\left(\mathrm{~d}, 2 \mathrm{H},{ }^{3} \mathrm{~J}_{\text {Hanti,Hmeso }}=8.3 \mathrm{~Hz}\right.$, allyl- $\left.\mathrm{H}_{\mathrm{anti}}\right)$; minor isomer: 9.07 $\left(\mathrm{m}, 2 \mathrm{H}\right.$, bpy-H6), $8.72\left(\mathrm{~d}, 2 \mathrm{H},{ }^{3} \mathrm{~J}_{\mathrm{H} 3, \mathrm{H} 4}=8.1 \mathrm{~Hz}, \mathrm{bpy}-\mathrm{H} 3\right), 8.28(\mathrm{dt}$, $2 \mathrm{H},{ }^{3} J_{\mathrm{H} 4, \mathrm{H} 3 / \mathrm{H} 5}=7.8 \mathrm{~Hz},{ }^{4} J_{\mathrm{H} 4, \mathrm{H} 6}=1.3 \mathrm{~Hz}$, bpy-H4), $7.82(\mathrm{t}, 2 \mathrm{H}$, ${ }^{3} J_{\mathrm{H} 5, \mathrm{H} 4 / \mathrm{H} 6}=6.4 \mathrm{~Hz}$, bpy-H5), $3.30\left(\mathrm{~m}, 2 \mathrm{H}\right.$, allyl- $\left.\mathrm{H}_{\text {syn }}\right), 3.05-3.14$ $\left(\mathrm{m}, 1 \mathrm{H}\right.$, allyl- $\left.\mathrm{H}_{\text {meso }}\right), 1.50\left(\mathrm{~d}, 2 \mathrm{H},{ }^{3} J_{\text {Hanti,Hmeso }}=8.0 \mathrm{~Hz}\right.$, allyl- $\left.\mathrm{H}_{\text {anti }}\right)$; ${ }^{13} \mathrm{C}$ NMR (DMSO- $d_{6}, 125.76 \mathrm{MHz}, \mathrm{ppm}$ ): $\delta$ major isomer: 220.52 (C三O), 154.17 (bpy-C2), 152.02 (bpy-C6), 139.36 (bpy-C4), 127.03 (bpy-C5), 123.44 (bpy-C3), 62.96 ( $\mathrm{CH}$-allyl), $45.91\left(\mathrm{CH}_{2}-\right.$ allyl); minor isomer: the signal intensity was below the detection limit.

[W( $\eta^{3}$-allyl)( $\left.\left.\mathbf{N}_{3}\right)(\mathbf{b p y})(\mathrm{CO})_{2}\right]$ (10). In a degassed mixture of methanol $(100 \mathrm{~mL})$ and acetone $(25 \mathrm{~mL})$, solid $\left[\mathrm{W}\left(\eta^{3}\right.\right.$. allyl)Cl(bpy)(CO) $)_{2}$ (9, $\left.300 \mathrm{mg}, 0.63 \mathrm{mmol}\right)$ and sodium azide (207 $\mathrm{mg}, 3.18 \mathrm{mmol}$ ) were dissolved under argon and the reaction mixture heated to reflux for $6 \mathrm{~h}$. The resulting precipitate was filtered off from the dark red solution, washed with water $(80 \mathrm{~mL})$ and methanol $(40 \mathrm{~mL})$, and dried under vacuum to result in a red-brown solid. Yield: $62 \%$ (189 mg, 0.39 mmol). Elemental analysis (\%): calc. $\mathrm{C}_{15} \mathrm{H}_{13} \mathrm{~N}_{5} \mathrm{O}_{2} \mathrm{~W}$ : $\mathrm{C} 37.60, \mathrm{H}$ 2.73, N 14.62, found: C 37.57, H 2.70, N 14.57; IR (ATR, $\mathrm{cm}^{-1}$ ): 2046 (vs), 1920 (s), 1821 (vs), 1602 (m), 1471 (m), 1442 (m), $756(\mathrm{~m}) ;{ }^{1} \mathrm{H}$ NMR (DMSO- $\left.d_{6}, 500.13 \mathrm{MHz}, \mathrm{ppm}\right): \delta$ major isomer: $8.84\left(\mathrm{ddd}, 2 \mathrm{H},{ }^{3} J_{\mathrm{H} 6, \mathrm{H} 5}=5.4 \mathrm{~Hz},{ }^{4} \mathrm{~J}_{\mathrm{H} 6, \mathrm{H} 4}=1.4 \mathrm{~Hz},{ }^{5} J_{\mathrm{H} 6, \mathrm{H} 3}=\right.$ $0.5 \mathrm{~Hz}$, bpy-H6), $8.64\left(\mathrm{~d}, 2 \mathrm{H},{ }^{3} J_{\mathrm{H} 3, \mathrm{H} 4}=8.2 \mathrm{~Hz}, \mathrm{bpy}-\mathrm{H} 3\right), 8.27(\mathrm{dt}$, $2 \mathrm{H},{ }^{3} J_{\mathrm{H} 4, \mathrm{H} 3 / \mathrm{H} 5}=7.9 \mathrm{~Hz},{ }^{4} J_{\mathrm{H} 4, \mathrm{H} 6}=1.6 \mathrm{~Hz}$, bpy-H4), 7.70 (ddd, $2 \mathrm{H}$, ${ }^{3} J_{\mathrm{H} 5, \mathrm{H} 4}=7.6 \mathrm{~Hz},{ }^{3} J_{\mathrm{H} 5, \mathrm{H} 6}=5.5 \mathrm{~Hz},{ }^{4} J_{\mathrm{H} 5, \mathrm{H} 3}=1.2 \mathrm{~Hz}$, bpy-H5), 2.95 (d, $2 \mathrm{H},{ }^{3} J_{\text {Hsyn, Hmeso }}=6.2 \mathrm{~Hz}$, allyl- $\left.\mathrm{H}_{\text {syn }}\right), 2.35-2.43(\mathrm{~m}, 1 \mathrm{H}$, allyl$\left.\mathrm{H}_{\text {meso }}\right), 1.48\left(\mathrm{~d}, 2 \mathrm{H},{ }^{3} J_{\text {Hanti,Hmeso }}=8.7 \mathrm{~Hz}\right.$, allyl- $\left.\mathrm{H}_{\text {anti }}\right)$; minor isomer: $9.07(\mathrm{~m}, 2 \mathrm{H}, \mathrm{bpy}-\mathrm{H} 6), 8.75\left(\mathrm{~d}, 2 \mathrm{H},{ }^{3} \mathrm{~J}_{\mathrm{H} 3, \mathrm{H} 4}=8.0 \mathrm{~Hz}\right.$, bpy$\mathrm{H} 3), 8.32\left(\mathrm{dt}, 2 \mathrm{H},{ }^{3} \mathrm{~J}_{\mathrm{H} 4, \mathrm{H} 3 / \mathrm{H} 5}=7.7 \mathrm{~Hz},{ }^{4} J_{\mathrm{H} 4, \mathrm{H} 6}=1.2 \mathrm{~Hz}\right.$, bpy$\mathrm{H} 4), 7.86\left(\mathrm{t}, 2 \mathrm{H},{ }^{3} \mathrm{~J}_{\mathrm{H} 5, \mathrm{H} 4 / \mathrm{H} 6}=6.2 \mathrm{~Hz}\right.$, bpy-H5), $3.18(\mathrm{~d}, 2 \mathrm{H}$, ${ }^{3} J_{\text {Hsyn,Hmeso }}=6.1 \mathrm{~Hz}$, allyl- $\left.\mathrm{H}_{\text {syn }}\right), 3.02-3.13\left(\mathrm{~m}, 1 \mathrm{H}\right.$, allyl- $\left.\mathrm{H}_{\text {meso }}\right)$, $1.51\left(\mathrm{~d}, 2 \mathrm{H},{ }^{3} \mathrm{~J}_{\text {Hanti,Hmeso }}=9.0 \mathrm{~Hz}\right.$, allyl- $\left.\mathrm{H}_{\text {anti }}\right) ;{ }^{13} \mathrm{C}$ NMR (DMSO- $d_{6}$, $125.76 \mathrm{MHz}, \mathrm{ppm}$ ): $\delta$ major isomer: 220.57 (C=O), 154.14 (bpyC2), 152.06 (bpy-C6), 139.70 (bpy-C4), 127.30 (bpy-C5), 123.44 (bpy-C3), 65.12 ( $\mathrm{CH}$-allyl), $47.91\left(\mathrm{CH}_{2}\right.$-allyl); minor isomer: the signal intensity was below the detection limit.

[W( $\eta^{3}$-allyl)(triazolate $\left.\left.{ }^{\mathrm{COOCH} 3, \mathrm{COOCH} 3}\right)(\mathrm{bpy})(\mathrm{CO})_{2}\right]$

(11).

$\left[\mathrm{W}\left(\eta^{3}\right.\right.$-allyl) $\left(\mathrm{N}_{3}\right)($ bpy $\left.)(\mathrm{CO})_{2}\right] \quad(\mathbf{1 0}, 30 \mathrm{mg}, 0.06 \mathrm{mmol})$ was dissolved in dichloromethane $(3 \mathrm{~mL})$. Dimethyl acetylenedicarboxylate (DMAD, $31 \mu \mathrm{L}, 35.6 \mathrm{mg}, 0.25 \mathrm{mmol}$ ) was added and the reaction mixture stirred at room temperature for $5 \mathrm{~d}$. Then, diethyl ether $(50 \mathrm{~mL})$ was added to precipitate the product from the red-brown solution. The resulting brown crystalline solid was filtered off, washed with diethyl ether (10 $\mathrm{mL}$ ), and dried under vacuum. Yield: $83 \%$ (33 $\mathrm{mg}, 0.05 \mathrm{mmol}$ ). Elemental analysis (\%): calc. $\mathrm{C}_{21} \mathrm{H}_{19} \mathrm{~N}_{5} \mathrm{O}_{6} \mathrm{~W}$ : $\mathrm{C} 40.60, \mathrm{H} 3.08, \mathrm{~N}$ 11.27, found: $39.87, \mathrm{H}$ 3.29, N 10.82; IR (ATR, $\mathrm{cm}^{-1}$ ): 1921 (s), 1841 (s), 1724 (s); ${ }^{1} \mathrm{H}$ NMR (DMSO- $d_{6}, 500.13 \mathrm{MHz}, \mathrm{ppm}$ ): $\delta$ major isomer: $8.82\left(\mathrm{dd}, 2 \mathrm{H},{ }^{3} J_{\mathrm{H} 6, \mathrm{H} 5}=5.5 \mathrm{~Hz},{ }^{4} J_{\mathrm{H} 6, \mathrm{H} 4}=0.9 \mathrm{~Hz}\right.$, bpy-H6), $8.61\left(\mathrm{~d}, 2 \mathrm{H},{ }^{3} \mathrm{~J}_{\mathrm{H} 3, \mathrm{H} 4}=8.2 \mathrm{~Hz}, \mathrm{bpy}-\mathrm{H} 3\right), 8.24(\mathrm{dt}, 2 \mathrm{H}$, ${ }^{3} J_{\mathrm{H} 4, \mathrm{H} 3 / \mathrm{H} 5}=7.9 \mathrm{~Hz},{ }^{4} J_{\mathrm{H} 4, \mathrm{H} 6}=1.5 \mathrm{~Hz}$, bpy-H4), 7.61 (ddd, $2 \mathrm{H},{ }^{3} J_{\mathrm{H} 5, \mathrm{H} 4}$ $=7.5 \mathrm{~Hz},{ }^{3} J_{\mathrm{H} 5, \mathrm{H} 6}=5.5 \mathrm{~Hz},{ }^{4} J_{\mathrm{H} 5, \mathrm{H} 3}=1.1 \mathrm{~Hz}$, bpy-H5), $3.63(\mathrm{~s}, 6 \mathrm{H}$, $\left.\mathrm{COOCH}_{3}\right), 3.10\left(\mathrm{~d}, 2 \mathrm{H},{ }^{3} J_{\text {Hsyn,Hmeso }}=6.2 \mathrm{~Hz}\right.$, allyl- $\left.\mathrm{H}_{\text {syn }}\right), 2.29-2.37$ $\left(\mathrm{m}, 1 \mathrm{H}\right.$, allyl- $\left.\mathrm{H}_{\text {meso }}\right), 1.68\left(\mathrm{~d}, 2 \mathrm{H},{ }^{3} J_{\text {Hanti,Hmeso }}=8.8 \mathrm{~Hz}\right.$, allyl- $\left.\mathrm{H}_{\text {anti }}\right)$; minor isomer: $3.59\left(\mathrm{~s}, \mathrm{COOCH}_{3}\right)$, the other signals were too weak for clear identification; ${ }^{13} \mathrm{C}$ NMR (DMSO- $d_{6}, 125.76 \mathrm{MHz}$, ppm): $\delta 220.04$ (C三O), $162.12\left(\mathrm{COOCH}_{3}\right), 155.53$ (bpy-C2), 152.50 (bpy-C6), 139.83 (bpy-C4), 138.83 (triazolate-C4/C5), 127.19 (bpy-C5), 123.15 (bpy-C3), 65.48 (CH-allyl), 51.79 $\left(\mathrm{COOCH}_{3}\right), 49.45\left(\mathrm{CH}_{2}\right.$-allyl); minor isomer: the signal intensity was below the detection limit.

[W( $\eta^{3}$-allyl)(triazolate $\left.\left.{ }^{\mathrm{CF}, \mathrm{COOEt}}\right)(\mathrm{bpy})(\mathrm{CO})_{2}\right]$

(12).

$\left[\mathrm{W}\left(\eta^{3}\right.\right.$-allyl) $\left(\mathrm{N}_{3}\right)($ bpy $\left.)(\mathrm{CO})_{2}\right] \quad(\mathbf{1 0}, 25 \mathrm{mg}, 0.05 \mathrm{mmol})$ was dissolved in dichloromethane $(5 \mathrm{~mL})$. 4,4,4-Trifluoro-2butynoic acid ethyl ester $(17 \mathrm{mg}, 0.10 \mathrm{mmol})$ was added and the reaction mixture stirred at room temperature for $6 \mathrm{~d}$. Then, diethyl ether $(30 \mathrm{~mL})$ was added to precipitate the product from the dark red solution. The resulting red-brown crystalline solid was filtered off, washed with diethyl ether (10 $\mathrm{mL}$ ), and dried under vacuum. Yield: $40 \%(12 \mathrm{mg}, 0.02 \mathrm{mmol}$ ). Elemental analysis (\%): calc. $\mathrm{C}_{21} \mathrm{H}_{18} \mathrm{~F}_{3} \mathrm{~N}_{5} \mathrm{O}_{4} \mathrm{~W}$ : C 39.09, $\mathrm{H} 2.81, \mathrm{~N}$ 10.85, found: 39.09, H 2.86, N 10.56; IR (ATR, $\mathrm{cm}^{-1}$ ): 1929 (s), 1850 (s), 1723 (s); ${ }^{1} \mathrm{H}$ NMR (DMSO- $d_{6}, 500.13 \mathrm{MHz}, \mathrm{ppm}$ ): $\delta$ major isomer: $8.83\left(\mathrm{dd}, 2 \mathrm{H},{ }^{3} J_{\mathrm{H} 6, \mathrm{H} 5}=5.4 \mathrm{~Hz},{ }^{4} J_{\mathrm{H} 6, \mathrm{H} 4}=0.8 \mathrm{~Hz}\right.$, bpy-H6), $8.64\left(\mathrm{~d}, 2 \mathrm{H},{ }^{3} J_{\mathrm{H} 3, \mathrm{H} 4}=8.2 \mathrm{~Hz}, \mathrm{bpy}-\mathrm{H} 3\right), 8.25(\mathrm{dt}, 2 \mathrm{H}$, ${ }^{3} J_{\mathrm{H} 4, \mathrm{H} 3 / \mathrm{H} 5}=7.9 \mathrm{~Hz},{ }^{4} J_{\mathrm{H} 4, \mathrm{H} 6}=1.6 \mathrm{~Hz}$, bpy-H4), 7.61 (ddd, $2 \mathrm{H},{ }^{3} J_{\mathrm{H} 5, \mathrm{H} 4}$ $\left.=7.5 \mathrm{~Hz},{ }^{3} J_{\mathrm{H} 5, \mathrm{H} 6}=5.5 \mathrm{~Hz},{ }^{4} J_{\mathrm{H} 5, \mathrm{H} 3}=1.1 \mathrm{~Hz}, \mathrm{bpy}-\mathrm{H} 5\right), 4.14\left(\mathrm{q}, 2 \mathrm{H},{ }^{3} \mathrm{~J}\right.$ $\left.=7.1 \mathrm{~Hz}, \mathrm{COOCH}_{2} \mathrm{CH}_{3}\right), 3.13\left(\mathrm{~d}, 2 \mathrm{H},{ }^{3} \mathrm{~J}_{\text {Hsyn, Hmeso }}=6.2 \mathrm{~Hz}\right.$, allyl$\left.\mathrm{H}_{\text {syn }}\right), 2.37-2.44\left(\mathrm{~m}, 1 \mathrm{H}\right.$, allyl- $\left.\mathrm{H}_{\text {meso }}\right), 1.71\left(\mathrm{~d}, 2 \mathrm{H},{ }^{3} J_{\text {Hanti,Hmeso }}=\right.$ $8.8 \mathrm{~Hz}$, allyl- $\left.\mathrm{H}_{\text {anti }}\right), 1.17\left(\mathrm{t}, 3 \mathrm{H},{ }^{3} \mathrm{~J}=7.1 \mathrm{~Hz}, \mathrm{COOCH}_{2} \mathrm{CH}_{3}\right)$; minor isomer: signals were too weak for clear identification; ${ }^{13} \mathrm{C} \mathrm{NMR}$ (DMSO- $d_{6}, 125.76 \mathrm{MHz}, \mathrm{ppm}$ ): $\delta$ major isomer: 219.88 (C三O), $159.69\left(\mathrm{COOCH}_{2} \mathrm{CH}_{3}\right), 155.70$ (bpy-C2), 152.45 (bpy-C6), 139.85 (bpy-C4), $137.07\left(\mathrm{q},{ }^{2} \mathrm{~J}_{\mathrm{C}, \mathrm{F}}=37.1 \mathrm{~Hz}\right.$, triazolate-C4), $136.18(\mathrm{~m}$, ${ }^{3} J_{C, F}<0.6 \mathrm{~Hz}$, triazolate-C5), 127.13 (bpy-C5), 123.09 (bpy-C3), $121.09\left(q,{ }^{1} J_{C, F}=268 \mathrm{~Hz}, C F_{3}\right), 65.39(\mathrm{CH}$-allyl), 60.39 $\left(\mathrm{COOCH}_{2} \mathrm{CH}_{3}\right), 49.50\left(\mathrm{CH}_{2}\right.$-allyl), $13.85\left(\mathrm{COOCH}_{2} \mathrm{CH}_{3}\right)$; minor isomer: the signal intensity was below the detection limit; ${ }^{19} \mathrm{~F}$ NMR (DMSO- $\left.d_{6}, 470.59 \mathrm{MHz}, \mathrm{ppm}\right): \delta-58.95\left(\mathrm{CF}_{3}\right.$, major species, $88 \%),-58.70\left(\mathrm{CF}_{3}\right.$, minor species, $\left.12 \%\right)$.

\section{$\mathrm{X}$-ray diffraction analysis}

Single crystals suitable for X-ray structure determination of $\mathbf{2}$, $5,6,11$, and 12 were obtained by slow diffusion of $n$-hexane into a solution of the compound in dichloromethane while $\mathbf{1 0}$ was recrystallized from a mixture of acetone and methanol. Selected crystals were immersed in a film of perfluoropolyether oil, mounted on a polyimide microloop (MicroMounts, MiTeGen) or glass fiber, and transferred to a stream of cold dinitrogen (Bruker Kryoflex2). Crystal data for 2, 6, 10, 11 and 12 were obtained on a BRUKER X8-APEX II while data for 5 was collected on a BRUKER SMART-APEX diffractometer. Both instruments were equipped with a CCD area detector and used graphite monochromated $\mathrm{Mo}_{\mathrm{K \alpha}}$ radiation. The structures were solved using the intrinsic 
phasing method, ${ }^{64}$ refined with the SHELXL program ${ }^{65}$ and expanded using Fourier techniques. All non-hydrogen atoms were refined anisotropically. Hydrogen atoms were included in structure factors calculations. All hydrogen atoms, except those of the allyl moiety in some cases, were assigned to idealised positions. The coordinates of the hydrogen atoms of the allyl moiety were refined freely. The $\mathrm{C}-\mathrm{H}$ distances in the allyl moiety were restrained to a values of 0.950 (5) $\AA$ during the refinement. Crystallographic data has been deposited with the Cambridge Crystallographic Data Centre as supplementary publications no. CCDC-1562700-1562705. These data can be obtained free of charge from the Cambridge Crystallographic Data Centre via www.ccdc.cam.ac.uk/data_request/cif.

\section{DFT calculations}

DFT calculations were carried out on the Linux cluster of the Leibniz-Rechenzentrum (LRZ) in Munich with ORCA version $2.8{ }^{66}$ using the BP86 functional with the resolution-of-theidentity (RI) approximation, a def2-TZVP/def2-TZVP/J basis set, the tightscf and grid4 options, and the COSMO solvation model with dimethylsulfoxide as the solvent for geometry optimization and subsequent calculation of vibrational frequencies to characterize the structure obtained as a minimum by inspection for absence of imaginary modes. Then, a relaxed surface scan was carried out in $15^{\circ}$ steps for the full $360^{\circ}$ rotation of the triazolate ring relative to the central C-C2 axis of the 2,2'-bipyridine ligand while allowing all other variables to relax. The maxima located on the resulting potential energy curve where then further characterized as transition states using the opt TS keyword in separate runs.

\section{Conflicts of interest}

There are no conflicts of interest to declare.

\section{Acknowledgements}

This work was supported by the Deutsche Forschungsgemeinschaft (DFG) with grants no. SCHA962/3-1 and SCHA962/8-1 to U.S.

\section{References}

1 H. C. Kolb, M. G. Finn and K. B. Sharpless, Angew. Chem. Int. Ed., 2001, 40, 2004.

2 H. C. Kolb and K. B. Sharpless, Drug Discovery Today, 2003, 8, 1128.

3 J. E. Moses and A. D. Moorhouse, Chem. Soc. Rev., 2007, 36, 1249 .

4 P. Thirumurugan, D. Matosiuk and K. Jozwiak, Chem. Rev., 2013, 113, 4905.

5 J. A. Prescher and C. R. Bertozzi, Nature Chem. Biol., 2005, $1,13$.

6 C. R. Bertozzi and E. M. Sletten, Angew. Chem. Int. Ed., 2009, 48, 6974.

7 R. K. V. Lim and Q. Lin, Chem. Commun., 2010, 46, 1589.
M. Grammel and H. C. Hang, Nature Chem. Biol., 2013, 9, 475.

C. P. Ramil and Q. Lin, Chem. Commun., 2013, 49, 11007 K. Lang and J. W. Chin, Chem. Rev., 2014, 114, 4764.

N. K. Devaraj and R. Weissleder, Acc. Chem. Res., 2011, 44, 816.

T. J. Del Castillo, S. Sarkar, K. A. Abboud and A. S. Veige, Dalton Trans., 2011, 40, 8140.

L. Cararrubios, M. C. de la Torre and M. A. Sierra, Chem. Eur. J., 2013, 19, 3534.

H.-W. Frühauf, Chem. Rev., 1997, 97, 523.

H.-W. Frühauf, Coord. Chem. Rev., 2002, 230, 79.

W. P. Fehlhammer and W. Beck, Z. Anorg. Allg. Chem., 2015, 641, 1599.

C. C. Beto, E. D. Holt, Y. Yang, I. Ghiviriga, K. S. Schanze and A. S. Veige, Chem. Commun., 2017, 53, 9934.

A. R. Powers, I. Ghiviriga, K. A. Abboud and A. S. Veige, Dalton Trans., 2015, 44, 14747.

L. Waag-Hiersch, J. Mößeler and U. Schatzschneider, Eur. J. Inorg. Chem., 2017, 3024.

J. A. Krause-Bauer, T. M. Becker and M. Orchin, J. Chem. Cryst., 2004, 34, 843.

K. Pachhunga, B. Therrien and M. R. Kollipara, Inorg. Chim. Acta, 2008, 361, 3294.

F.-C. Liu, Y.-L. Lin, P.-S. Yang, G.-H. Lee and S.-M. Peng, Organometallics, 2010, 29, 4282.

K. Agapiou, R. A. Jones and X. Yang, Main Group Chem., 2010, 9, 41.

Y.-H. Lo, T.-H. Wang, C.-Y. Lee and Y.-H. Feng, Organometallics, 2012, 31, 6887.

S. Miguel-Fernandez, S. M. de Salinas, J. Diez, M. P. Gamasa and E. Lastra, Inorg. Chem., 2013, 52, 4293.

F.-C. Liu, J.-E. Liang, J.-Y. Jin, Y.-L. Lin, Y.-J. Chu, P.-S. Yang, G.-H. Lee and S.-M. Peng, J. Organomet. Chem., 2013, 735, 1.

T. Cruchter, K. Harms and E. Meggers, Chem. Eur. J., 2013, 19, 16682.

L. Henry, C. Schneider, B. Mützel, P. V. Simpson, C. Nagel, K. Fucke and U. Schatzschneider, Chem. Commun., 2014, 50, 15692.

K. S. Singh, K. A. Kreisel, G. P. A. Yap and M. R. Kollipara, J. Organomet. Chem., 2006, 691, 3509.

H. Pfeiffer, M. Dragoun, A. Prokop and U. Schatzschneider, Z. Anorg. Allg. Chem., 2013, 639, 1568.

T. S. A. Hor and S.-M. Chee, J. Organomet. Chem., 1987, 331, 23.

H. Behrens and N. Harder, Chem. Ber., 1964, 97, 433.

S. S. van Berkel, T. J. Dirks, M. F. Debets, F. L. van Delft, J. J. L. M. Cornelissen, R. J. M. Nolte and F. P. J. T. Rutjes, ChemBioChem, 2007, 8, 1504.

J. E. Bunch and C. L. Bumgardner, J. Fluorine Chem., 1987, 36, 313.

B. C. Hamper, Org. Synth., 1992, 70, 246.

Y. Kobayashi, T. Yamashita, K. Takahashi, H. Kuroda and I. Kumadaki, Tetrahedron Lett., 1982, 23, 343.

G. Meazza and G. Zanardi, J. Fluorine Chem., 1991, 55, 199.

S. T. D. Gough and S. Trippett, J. Chem. Soc., 1962, 2333.

Y. Kobayashi, T. Yamashita, K. Takahashi, H. Kuroda and I. Kumadaki, Chem. Pharm. Bull., 1984, 32, 4402.

F. Eymery, B. lorga and P. Savignac, Synthesis, 2000, 185.

Z. Dori and R. F. Ziolo, Chem. Rev., 1973, 73, 247. 
42 J. W. Faller, D. A. Haitko, R. D. Adams and D. F. Chodosh, J. Am. Chem. Soc., 1979, 101, 865.

43 J. W. Faller and B. C. Whitmore, Organometallics, 1986, 5, 752.

44 M. J. Calhorda and P. J. Costa, Coord. Chem. Rev., 2017, 344, 83.

45 D. E. Ryan, D. J. Cardin and F. Hartl, Coord. Chem. Rev., 2017, 335, 103.

46 A. G. Orpen, L. Brammer, F. H. Allen, O. Kennard, D. G. Watson and R. Taylor, Dalton Trans., 1989, S1.

47 E. O. Fischer, D. Wittmann, D. Himmelreich, R. Cai, K. Ackermann and D. Neugebauer, Chem. Ber., 1982, 115, 3152.

48 D. Sellmann, W. Weber, G. Liehr and H. P. Beck, J. Organomet. Chem., 1984, 269, 155.

49 H. Wadle, E. Conradi, U. Müller and K. Dehnicke, Z. Naturforsch. B, 1985, 40, 1626.

50 D. Fenske, A. Frankenau and K. Dehnicke, Z. Anorg. Allg. Chem., 1989, 579, 27.

51 H.-W. Lam, G. Wilkinson, B. Hussian-Bates and M. B. Hursthouse, Dalton Trans., 1993, 781.

52 D. Rische, A. Baunemann, M. Winter and R. A. Fischer, Inorg. Chem., 2006, 45, 269.

53 F.-C. Liu, T.-C. Tsai, Y.-L. Lin, C.-S. Lee, P.-S. Yang and J.-C. Wang, J. Organomet. Chem., 2010, 695, 423.

54 R. Haiges, J. Skotnitzki, Z. Fang, D. A. Dixon and K. O. Christe, Angew. Chem. Int. Ed., 2015, 54, 9581.

55 R. Haiges, J. Skotnitzki, Z. Fang, D. A. Dixon and K. O. Christe, Angew. Chem. Int. Ed., 2015, 54, 15550.

56 J. Pérez, V. Riera, A. Rodríguez, R. López, T. L. Sordo, S. García-Granda, E. García-Rodríguez and A. Galindo, Organometallics, 2003, 22, 1540.

57 K.-B. Shiu, S.-T. Lin, D.-W. Fung, T.-J. Chan, S.-M. Peng, M.C. Cheng and J. L. Chou, Inorg. Chem., 1995, 34, 854.

58 C. Hansch, A. Leo and R. W. Taft, Chem. Rev., 1991, 91, 165.

59 A. R. Powers, X. Yang, T. J. Del Castillo, I. Ghiviriga, K. A. Abboud and A. S. Veige, Dalton Trans., 2013, 42, 14963.

60 E. Evangelino, N. P. Rath and L. M. Mirica, Dalton Trans., 2012, 41, 8010.

61 E. A. Giner, M. Gomez-Gallego, L. Casarrubios, M. C. de la Torre, C. R. de Arellano and M. A. Sierra, Inorg. Chem., 2017, 56, 2801.

62 E. Hirota, S. Saito and Y. Endo, J. Chem. Phys., 1979, 71, 1183.

63 G. R. Fulmer, A. J. M. Miller, N. H. Sherden, H. E. Gottlieb, A. Nudelman, B. M. Stoltz, J. E. Bercaw and K. I. Goldberg, Organometallics, 2010, 29, 2176.

64 G. M. Sheldrick, Acta Cryst. A, 2015, A71, 3.

65 G. M. Sheldrick, Acta Cryst. A, 2008, A64, 112.

66 F. Neese, WIREs Comput. Mol. Sci., 2011, 2, 73. 